\title{
Asymmetric Total Synthesis of (+)-Quinocarcinamide
}

\author{
Lei Li, ${ }^{\text {a,b }}$ Li Shi, ${ }^{\text {a,b }}$ Kun Wei, ,a and Yu-Rong Yang ${ }^{*}, \mathrm{a}$ \\ ${ }^{a}$ State Key Laboratory of Phytochemistry and Plant Resources in West China, Kunming Institute of Botany, \\ Chinese Academy of Sciences, Kunming 650201, China \\ ${ }^{b}$ University of Chinese Academy of Sciences, Beijing 100049, China \\ Email: yangyurong@mail.kib.ac.cn \\ Email:weikun@mail.kib.ac.cn
}

\section{Table of Contents}

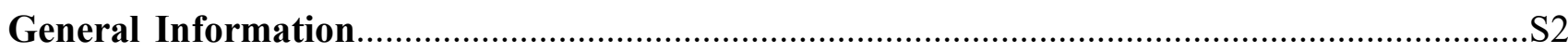

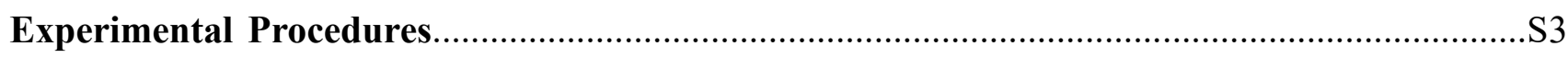

Synthetic Procedures and Characterization Data.................................................................S3

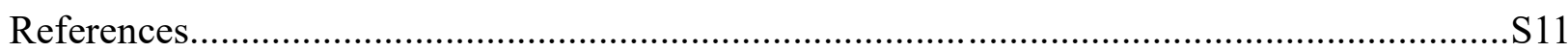

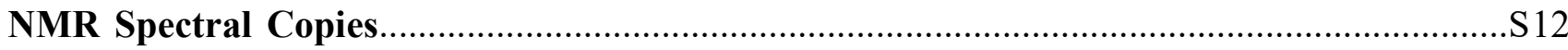

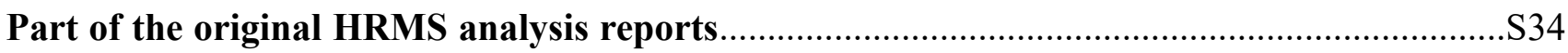

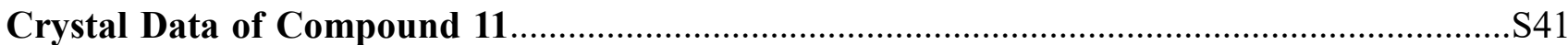




\section{General Information}

Unless otherwise stated, all oxygen or moisture sensitive reactions were conducted in flame-dried glassware under an atmosphere of nitrogen. All solvents were purified and dried according to standard methods prior to use. ( $S$ )-L and ( $\mathrm{rac}$ )-L were prepared according to the reported procedure. ${ }^{1}$ Reagents were purchased from commercial sources and were used without further purification.

Chromatographic purification of products was accomplished using forced-flow chromatography on 200-300 mesh silica gel supplied by Tsingtao Haiyang Chemicals (China). The TLC glass plates were performed on $0.20 \mathrm{~mm}$ silica gel GF254 plates supplied by Yantai Chemicals (China), and visualized with UV light ( $254 \mathrm{~nm}$ ), exposure to iodine vapor, or basic aqueous potassium permanganate $\left(\mathrm{KMnO}_{4}\right)$.

${ }^{1} \mathrm{H}$ and ${ }^{13} \mathrm{C}$ NMR spectra were acquired on Bruker Avance III 400,500 and 600 NMR spectrometer. Chemical shifts were given in parts per million (ppm) with reference to residual solvent signals $\left[{ }^{1} \mathrm{H}\right.$ NMR: $\mathrm{CDCl}_{3}$ (7.26), ${ }^{13} \mathrm{C} \mathrm{NMR}: \mathrm{CDCl}_{3}$ (77.16); ${ }^{1} \mathrm{H} \mathrm{NMR}:\left(\mathrm{CD}_{3}\right)_{2} \mathrm{CO}(2.05),{ }^{13} \mathrm{C} \mathrm{NMR}:\left(\mathrm{CD}_{3}\right)_{2} \mathrm{CO}$ : (29.90)]. Peak multiplicities were recorded as follows: $\mathrm{s}=$ singlet, $\mathrm{d}=$ doublet, $\mathrm{t}=$ triplet, $\mathrm{q}=$ quartet, $\mathrm{m}=$ multiplet, $\mathrm{br}=$ broad singlet. Infrared (IR) spectra were recorded on a BRUKER Tensor-27 Fourier-Transform Infrared spectrometer. High resolution mass spectral (HRMS) data were obtained at the mass spectrometry service operated at Agilent 6540 Q-TOF spectrometer and a Shimadzu UPLCIT-TOF spectrometer for electrospray ionization (ESI) and were reported as $(\mathrm{m} / \mathrm{z})$. X-ray diffraction was conducted using Bruker APEX DUO diffractometer with graphite-monochromated $\mathrm{CuK \alpha}$ radiation. Optical rotations were measured on a Jasco P-1020 polarimeter. Melting points were measured on a WRX-5A melting point apparatus. HPLC analysis was performed on an Agilent 1260 series system using Daicel Chiralpak AD-H with $n$-hexane and $i-\mathrm{PrOH}$ as solvents. 


\section{Experimental Procedures}

\section{Synthetic Procedures and Characterization Data}<smiles>COc1cccc(Br)c1C=O</smiles>

10

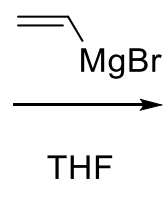<smiles>C=CC(O)c1c(Br)cccc1OC</smiles>

9

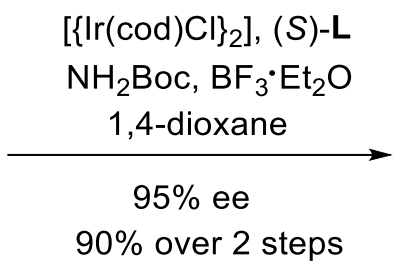

$90 \%$ over 2 steps

2-Bromo-6-methoxybenzaldehyde 10 (2.15 g, $10.0 \mathrm{mmol}, 1.0$ equiv) was dissolved in dry THF $(30 \mathrm{~mL})$ and cooled down to $0^{\circ} \mathrm{C}$. Vinyl magnesium bromide $(15 \mathrm{~mL}, 1.0 \mathrm{M}$ in THF, $15.0 \mathrm{mmol}, 1.5$ equiv) was added to the mixture dropwise. The reaction was left stirring for $10 \mathrm{~min}$ at $0{ }^{\circ} \mathrm{C}$ and quenched with saturated aqueous $\mathrm{NH}_{4} \mathrm{Cl}(30 \mathrm{~mL})$. The mixture was extracted with ethyl acetate $(2 \times$ $50 \mathrm{~mL}$ ) and the combined organic phase was washed with brine, dried over anhydrous $\mathrm{Na}_{2} \mathrm{SO}_{4}$. The dried solution was concentrated in vacuo. The crude residue 9 was used in the subsequent step without further purification.

A flame-dried $100 \mathrm{~mL}$ round-bottom flask equipped with a stir bar was charged with $[\operatorname{Ir}(\operatorname{cod}) \mathrm{Cl}]_{2}$ (201 mg, $0.3 \mathrm{mmol}, 3 \mathrm{~mol} \%),(S)$-L (611 mg, $1.2 \mathrm{mmol}, 12 \mathrm{~mol} \%)$ and 1,4-dioxane (15 mL) under an atmosphere of nitrogen. After vigorous stirring for $10 \mathrm{~min}$ at room temperature, a solution of the crude product 9 in $5 \mathrm{~mL}$ 1,4-dioxane obtained in the prior transformation, BocNH $\mathrm{N}_{2}(1.76 \mathrm{~g}, 15.0 \mathrm{mmol}, 1.5$ equiv), and promoter $\mathrm{BF}_{3} \cdot \mathrm{Et}_{2} \mathrm{O}(252 \mu \mathrm{L}, 2.0 \mathrm{mmol}, 20 \mathrm{~mol} \%)$ were added sequentially to the resulting solution. The reaction mixture was stirred for $1.5 \mathrm{~h}$ at room temperature and then quenched with saturated aqueous $\mathrm{NaHCO}_{3}(5 \mathrm{~mL})$. The mixture was extracted with ethyl acetate $(2 \times 50 \mathrm{~mL})$ and the combined organic phase was washed with brine, dried over anhydrous $\mathrm{Na}_{2} \mathrm{SO}_{4}$. The dried solution was concentrated in vacuo. The crude residue was purified by silica gel flash chromatography (petroleum ether/ethyl acetate $=5: 1)$ to afford the desired product $\mathbf{8}(3.07 \mathrm{~g}, 90 \%$ yield, 2 steps $)$ as a yellow oil.

Data for compound 9

Compound 9 was isolated for characterization purposes by purifying a small amount of crude by column chromatography (petroleum ether/ethyl acetate $=5: 1$ ). $\mathbf{R}_{\boldsymbol{f}}=0.48$ (petroleum ether/ethyl acetate $=4: 1) .{ }^{1} \mathbf{H}$ NMR $\left(400 \mathrm{MHz}, \mathrm{CDCl}_{3}\right) \delta 7.19(\mathrm{~d}, J=8.0 \mathrm{~Hz}, 1 \mathrm{H}), 7.08(\mathrm{t}, J=8.1 \mathrm{~Hz}, 1 \mathrm{H}), 6.86(\mathrm{~d}, J=$ $8.2 \mathrm{~Hz}, 1 \mathrm{H}), 6.15$ (ddd, $J=17.1,10.3,5.4 \mathrm{~Hz}, 1 \mathrm{H}), 5.77-5.56(\mathrm{~m}, 1 \mathrm{H}), 5.24$ (dt, $J=17.2,1.5 \mathrm{~Hz}, 1 \mathrm{H})$, $5.12(\mathrm{dd}, J=10.4,1.6 \mathrm{~Hz}, 1 \mathrm{H}), 4.01(\mathrm{~d}, J=22.6 \mathrm{~Hz}, 1 \mathrm{H}), 3.86(\mathrm{~s}, 3 \mathrm{H}) \mathrm{ppm} .{ }^{13} \mathrm{C}$ NMR $(100 \mathrm{MHz}$, $\left.\mathrm{CDCl}_{3}\right) \delta 158.3,138.7,129.7,129.3,125.9,123.5,114.6,110.8,74.9,56.0$ ppm. IR (KBr): 3545, 3082, 2847, 1588, 1571, 1538, 1462, 1434, 1318, 1260, 1231, 1179, 1028, 927, 866, 799, 703, $622 \mathrm{~cm}^{-}$ ${ }^{1}$. HRMS (ESI-TOF) $\mathbf{m} / \mathbf{z}:[\mathrm{M}+\mathrm{Na}]^{+}$Calcd for $\mathrm{C}_{10} \mathrm{H}_{11} \mathrm{BrO}_{2} \mathrm{Na} 264.9835$; Found 264.9831 .

Data for compound $\mathbf{8}$

$\mathbf{R}_{\boldsymbol{f}}=0.67$ (petroleum ether/ethyl acetate $\left.=5: 1\right) .{ }^{1} \mathbf{H} \mathbf{~ N M R}\left(400 \mathrm{MHz}, \mathrm{CDCl}_{3}\right) \delta 7.02(\mathrm{~d}, J=7.9$ $\mathrm{Hz}, 1 \mathrm{H}), 6.92(\mathrm{t}, J=8.1 \mathrm{~Hz}, 1 \mathrm{H}), 6.68(\mathrm{~d}, J=8.2 \mathrm{~Hz}, 1 \mathrm{H}), 5.99(\mathrm{dd}, J=10.2,5.4 \mathrm{~Hz}, 1 \mathrm{H}), 5.86(\mathrm{ddd}$, $J=16.6,10.2,4.5 \mathrm{~Hz}, 2 \mathrm{H}), 5.03(\mathrm{~d}, J=17.1 \mathrm{~Hz}, 1 \mathrm{H}), 4.93(\mathrm{~d}, J=10.1 \mathrm{~Hz}, 1 \mathrm{H}), 3.65(\mathrm{~s}, 3 \mathrm{H}), 1.32(\mathrm{~s}$, 9H) ppm. ${ }^{13} \mathbf{C ~ N M R}\left(100 \mathrm{MHz}, \mathrm{CDCl}_{3}\right) \delta 158.1,154.6,136.7,129.0,128.2,125.2,123.8,114.2,110.5$, 78.8, 55.6, 54.6, 28.1 ppm. IR (KBr): 3445, 3090, 3003, 2972, 2846, 1701, 1640, 1571, 1494, 1416, 1336, 1289, 1227, 1075, 989, 879, 803, 734, 693, $601 \mathrm{~cm}^{-1}$. HRMS (ESI-TOF) m/z: $[\mathrm{M}+\mathrm{Na}]^{+} \mathrm{Calcd}$ for $\mathrm{C}_{15} \mathrm{H}_{20} \mathrm{BrNO}_{3} \mathrm{Na} 364.0519$; Found 364.0519. $[\alpha]_{\mathrm{D}}^{21.5}=-23.11^{\circ}\left(c=0.56, \mathrm{CHCl}_{3}\right)$.

HPLC analysis indicated that the enantiomeric excess of the product was $95 \%$.

HPLC $($ AD-H, $i-\mathrm{PrOH} / n$-hexane $=5 / 95$, flow rate $=1.0 \mathrm{~mL} / \mathrm{min}, \mathrm{I}=254 \mathrm{~nm}) \mathrm{tR}=6.775 \mathrm{~min}$ (major) ,7.768 min (minor). 
Racemicte compound $\mathbf{8}$ was acquired by using the above procedure with racemic ligand ( $\mathrm{rac}$ )-L instead of $(S)$-L.<smiles>C=CC(NC(C)(C)C)c1c(Br)cccc1OC</smiles>

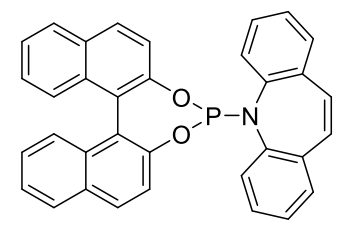

(rac)-L

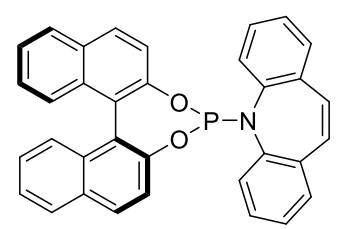

$(s)-\mathbf{L}$

$(-)-8$

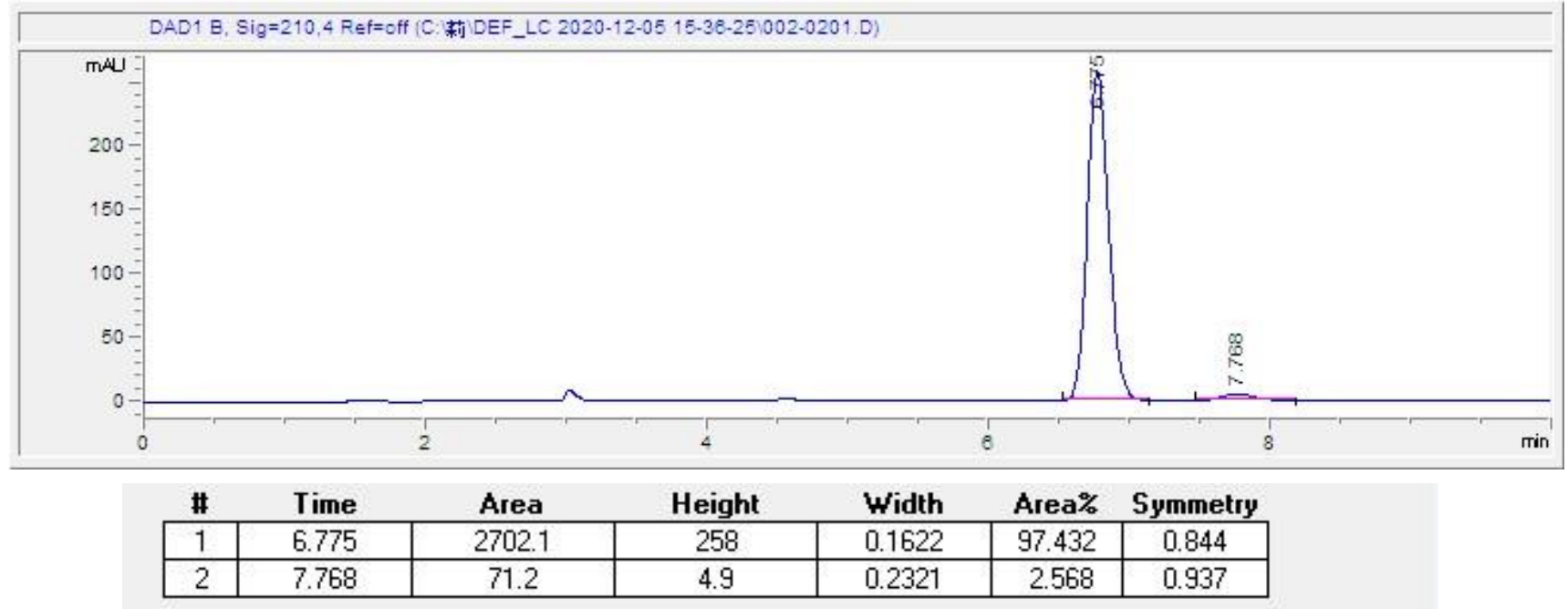

$r a c \mathbf{- 8}$

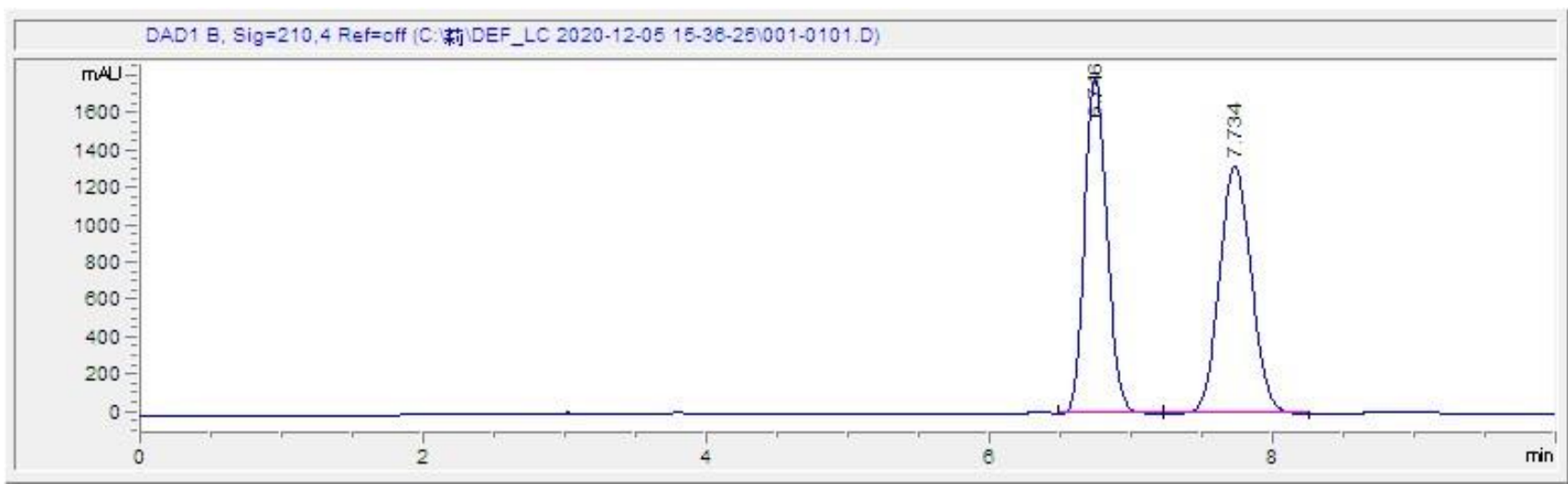

\begin{tabular}{|c|c|c|c|c|c|c|}
\hline$\#$ & Time & Area & Height & Width & Area\% & Symmetry \\
\hline 1 & 6.746 & 19754.2 & 1787.6 & 0.1729 & 48.769 & 0.829 \\
\hline 2 & 7.734 & 20751.8 & 1322.8 & 0.247 & 51.231 & 0.906 \\
\hline
\end{tabular}



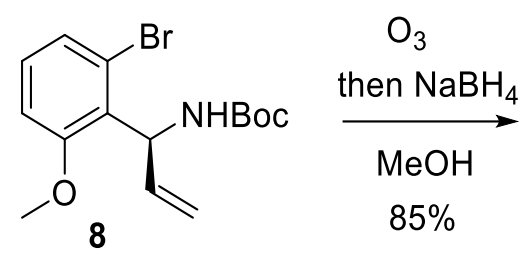<smiles>COc1cccc(Br)c1[C@H](CO)NC(C)(C)C</smiles>

Compound 8 (1.0 g, $2.92 \mathrm{mmol}, 1.0$ equiv) was dissolved in dry DCM (32 mL) and cooled down to $-78{ }^{\circ} \mathrm{C}$. An $\mathrm{O}_{3}-\mathrm{O}_{2}$ stream was passed through the solution for $15 \mathrm{~min}$. The solution was purged with nitrogen for $15 \mathrm{~min}$, and then $\mathrm{MeOH}(15 \mathrm{~mL})$ and $\mathrm{NaBH}_{4}(552.6 \mathrm{mg}, 14.6 \mathrm{mmol}, 5.0$ equiv) was added and the suspension was stirred at $0{ }^{\circ} \mathrm{C}$ for $4 \mathrm{~h}$. The mixture was quenched with saturated aqueous $\mathrm{NH}_{4} \mathrm{Cl}(10 \mathrm{~mL})$ and was warmed to room temperature. The mixture was extracted with ethyl acetate $(2 \times 50 \mathrm{~mL})$. The combined organic layers were washed with brine, dried over anhydrous $\mathrm{Na}_{2} \mathrm{SO}_{4}$. The dried solution was concentrated in vacuo. The crude product was purified by silica gel flash chromatography (petroleum ether/ethyl acetate $=2: 1)$ to afford the desired product $11(858 \mathrm{mg}, 85 \%$ yield) as a white solid. $\mathbf{R}_{\boldsymbol{f}}=0.23$ (petroleum ether/ethyl acetate $\left.=2: 1\right)$. m.p.: $58-60{ }^{\circ} \mathrm{C} .{ }^{\mathbf{1}} \mathbf{H} \mathbf{~ N M R}(400$ $\left.\mathrm{MHz} \mathrm{CDCl}_{3}\right) \delta 7.09(\mathrm{~d}, J=7.9 \mathrm{~Hz}, 1 \mathrm{H}), 6.99(\mathrm{t}, J=8.0 \mathrm{~Hz}, 1 \mathrm{H}), 6.75(\mathrm{~d}, J=8.3 \mathrm{~Hz}, 1 \mathrm{H}), 5.91(\mathrm{~s}$, $1 \mathrm{H}), 5.61-5.51(\mathrm{~m}, 1 \mathrm{H}), 3.77(\mathrm{~s}, 3 \mathrm{H}), 3.76(\mathrm{~d}, J=12.0 \mathrm{~Hz}, 1 \mathrm{H}), 3.66(\mathrm{~d}, J=12.0 \mathrm{~Hz}, 1 \mathrm{H}), 3.25(\mathrm{~s}$, $1 \mathrm{H}), 1.36(\mathrm{~s}, 9 \mathrm{H}) \mathrm{ppm} .{ }^{13} \mathbf{C} \mathbf{N M R}\left(100 \mathrm{MHz}, \mathrm{CDCl}_{3}\right) \delta 158.6,156.1,129.3,126.5,125.6,124.9,110.5$, 79.3, 63.8, 55.7, 55.4, 28.2 ppm. IR (KBr): 3466, 3068, 2964, 2881, 2722, 2532, 1695, 1574, 1500, 1436, 1379, 1284, 1155, 1023, 955,801, 730, $556 \mathrm{~cm}^{-1}$. HRMS (ESI-TOF) m/z: $[\mathrm{M}+\mathrm{Na}]^{+}$Calcd for $\mathrm{C}_{14} \mathrm{H}_{20} \mathrm{BrNO}_{4} \mathrm{Na} 368.0468$; Found 368.0476. $[\boldsymbol{\alpha}]_{\mathrm{D}}^{20.8}=-12.18^{\circ}\left(c=0.98, \mathrm{CHCl}_{3}\right)$. A sample of compound $\mathbf{1 1}$ for X-ray analysis was recrystallized from n-hexane/ ethyl acetate (3:1) via slow evaporation to afford colorless needles.<smiles>COC(=O)N[C@@H](CO)c1c(Br)cccc1OC</smiles>

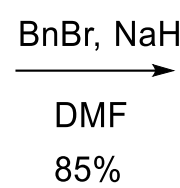

$85 \%$<smiles>COc1cccc(Br)c1C(COc1ccccc1)NC(=O)OCc1ccccc1</smiles>

12

Sodium hydride, $60 \%$ dispersion in mineral oil ( $110 \mathrm{mg}, 2.73 \mathrm{mmol}, 1.1$ equiv) was added to a well-stirred solution of the compound $11\left(2.48 \mathrm{mmol}, 858 \mathrm{mg}, 1.0\right.$ equiv) in dry DMF $(15 \mathrm{~mL})$ at $0{ }^{\circ} \mathrm{C}$ under argon. After $10 \mathrm{~min}$, a solution of the benzyl bromide (470 $\mathrm{mg}, 2.73 \mathrm{mmol}, 1.1$ equiv) in DMF $(5 \mathrm{~mL})$ was added dropwise. The reaction was left stirring at $0{ }^{\circ} \mathrm{C}$ for $2 \mathrm{~h}$. The reaction mixture was then poured on ice, followed by addition of $\mathrm{HCl}(2 \mathrm{~N}, 5 \mathrm{~mL})$, and the mixture was extracted with ethyl acetate $(2 \times 50 \mathrm{~mL})$. The organic layers were combined and were washed with brine. The combined organic phase was dried over anhydrous $\mathrm{Na}_{2} \mathrm{SO}_{4}$. The dried solution was concentrated in vacuo. The residue was purified by flash chromatography (petroleum ether/ethyl acetate $=2: 1$ ) to give the pure product 12 (919 $\mathrm{mg}, 85 \%$ yield) as a colorless oil. $\mathbf{R}_{\boldsymbol{f}}=0.6$ (petroleum ether/ethyl acetate $\left.=2: 1\right) .{ }^{1} \mathbf{H}$ NMR $\left(400 \mathrm{MHz}, \mathrm{CDCl}_{3}\right) \delta$ 7.25-7.26. $(\mathrm{m}, 5 \mathrm{H}), 7.18-7.05(\mathrm{~m}, 1 \mathrm{H}), 7.01(\mathrm{~d}, J=3.4 \mathrm{~Hz}, 1 \mathrm{H}), 6.73(\mathrm{dd}$, $J=8.1,4.2 \mathrm{~Hz}, 1 \mathrm{H}), 5.95-5.74(\mathrm{~m}, 2 \mathrm{H}), 4.62(\mathrm{~d}, J=11.9 \mathrm{~Hz}, 1 \mathrm{H}), 4.52(\mathrm{~d}, J=11.1 \mathrm{~Hz}, 1 \mathrm{H}), 3.80-$ $3.73(\mathrm{~m}, 1 \mathrm{H}), 3.70(\mathrm{~s}, 3 \mathrm{H}), 3.60(\mathrm{dd}, J=10.6,5.8 \mathrm{~Hz}, 1 \mathrm{H}), 1.42(\mathrm{~s}, 9 \mathrm{H}) \mathrm{ppm} .{ }^{13} \mathrm{C}$ NMR $(100 \mathrm{MHz}$, $\left.\mathrm{CDCl}_{3}\right) \delta 158.8,155.4,138.4,129.4,128.1,127.6,127.4,127.4,126.9,125.6,125.0,110.5,79.2,72.2$, 70.6, 55.7, 52.3, 28.4 ppm. IR (KBr): 3455, 3088, 3003, 2975, 2861, 1714, 1588, 1460, 1500, 1390, 1310, 1258, 1169, 1030, 906,851, 778, 526 $\mathrm{cm}^{-1}$. HRMS (ESI-TOF) $\mathbf{m} / \mathbf{z}:[\mathrm{M}+\mathrm{Na}]^{+}$Calcd for $\mathrm{C}_{21} \mathrm{H}_{26} \mathrm{BrNO}_{4} \mathrm{Na}$ 458.0937; Found 458.0947. $[\alpha]_{\mathrm{D}}^{21.3}=+7.44^{\circ}\left(c=0.59, \mathrm{CHCl}_{3}\right)$. 

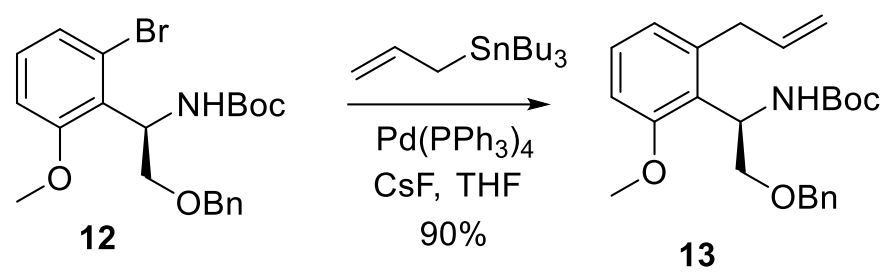

To a solution of compound $12(1.0 \mathrm{~g}, 2.3 \mathrm{mmol}, 1.0$ equiv) in dry THF (30 mL) was added successively allyltributyltin ( $837 \mathrm{mg}, 27.8 \mathrm{mmol}, 1.1$ equiv), $\mathrm{Pd}\left(\mathrm{PPh}_{3}\right)_{4}(132 \mathrm{mg}, 0.12 \mathrm{mmol}, 5 \mathrm{~mol} \%)$, powdered CsF ( $871 \mathrm{mg}, 5.8 \mathrm{mmol}, 2.5$ equiv). The resulting suspension was degassed under a stream of nitrogen for $0.5 \mathrm{~h}$, and then heated to reflux in an oil bath and stirred for $8 \mathrm{~h}$. The mixture was cooled down to room temperature, then quenched with $\mathrm{H}_{2} \mathrm{O}(30 \mathrm{~mL})$. The mixture was extracted with ethyl acetate $(2 \times 100 \mathrm{~mL})$. The organic layers were combined and were washed with brine $(3 \times 10 \mathrm{~mL})$, dried over anhydrous $\mathrm{Na}_{2} \mathrm{SO}_{4}$. The dried solution was concentrated in vacuo. The crude residue was purified by silica gel flash chromatography (petroleum ether/ethyl acetate $=5: 1$ to $4: 1$ ) to afford the desired product $13\left(819 \mathrm{mg}, 90 \%\right.$ yield) as a colorless oil. $\mathbf{R}_{\boldsymbol{f}}=0.55$ (petroleum ether/ethyl acetate $=$ 5:1). ${ }^{1}$ H NMR $\left(400 \mathrm{MHz}\right.$, Acetone- $\left.\mathrm{d}_{6}\right) \delta 7.34-7.20(\mathrm{~m}, 5 \mathrm{H}), 7.18(\mathrm{t}, J=7.9 \mathrm{~Hz}, 1 \mathrm{H}), 6.89(\mathrm{~d}, J=8.3$ $\mathrm{Hz}, 1 \mathrm{H}), 6.80(\mathrm{~d}, J=7.6 \mathrm{~Hz}, 1 \mathrm{H}), 6.05(\mathrm{ddd}, J=20.6,10.6,7.3 \mathrm{~Hz}, 2 \mathrm{H}), 5.41(\mathrm{td}, J=8.8,6.0 \mathrm{~Hz}, 1 \mathrm{H})$, $5.09-4.85(\mathrm{~m}, 2 \mathrm{H}), 4.50(\mathrm{q}, J=12.0 \mathrm{~Hz}, 2 \mathrm{H}), 3.90(\mathrm{t}, J=9.1 \mathrm{~Hz}, 1 \mathrm{H}), 3.83(\mathrm{~s}, 3 \mathrm{H}), 3.67(\mathrm{dd}, J=15.6$, $7.1 \mathrm{~Hz}, 1 \mathrm{H}), 3.60(\mathrm{dd}, J=9.8,6.1 \mathrm{~Hz}, 1 \mathrm{H}), 3.50(\mathrm{dd}, J=15.7,5.8 \mathrm{~Hz}, 1 \mathrm{H}), 1.38(\mathrm{~s}, 9 \mathrm{H}) \mathrm{ppm} .{ }^{13} \mathrm{C}$ NMR $(150 \mathrm{MHz}$, Acetone-d 6 ) $\delta$ 159.6, 156.2, 140.3, 139.9, 138.4, 129.3, 129.0, 128.3, 128.1, 127.2, 123.5, 116.0, 110.6, 78.9, 73.0, 72.5, 55.8, 49.9, 38.8, 28.7 ppm. IR (KBr): 3456, 3029, 2975, 2858, $1711,1599,1497,1455,1406,1390,1364,1170,1053,997,870,742,616,583 \mathrm{~cm}^{-1}$. HRMS (ESITOF $) \mathbf{m} / \mathbf{z}:[\mathrm{M}+\mathrm{H}]^{+}$Calcd for $\mathrm{C}_{24} \mathrm{H}_{32} \mathrm{NO}_{4}$ 398.2326;Found 398.2331. $[\boldsymbol{\alpha}]_{\mathrm{D}}{ }^{20.4}=-33.95^{\circ}(c=0.83$, $\left.\mathrm{CHCl}_{3}\right)$.

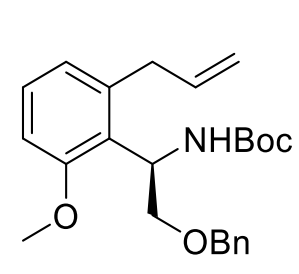

13

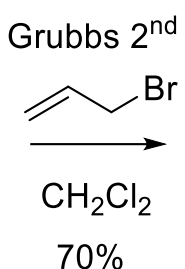

$70 \%$<smiles>COc1cccc(C/C=C/CBr)c1[C@H](N)COC(=O)Br</smiles>

7

Compound 13 (200 mg, $0.50 \mathrm{mmol}, 1.0$ equiv), allyl bromide ( $302.5 \mathrm{mg}, 2.5 \mathrm{mmol}, 5.0$ equiv), Grubbs' second generation catalyst $(42.5 \mathrm{mg}, 0.05 \mathrm{mmol}, 10 \mathrm{~mol} \%)$ and DCM (5 mL, 0.1M) were sequentially added into a pressure tube equipped with a magnetic stir bar. The tube was purged with argon for 1 minute. The tube was sealed with a PTFE lined cap and placed in an oil bath at $40{ }^{\circ} \mathrm{C}$ and stirred for $12 \mathrm{~h}$. After cooling back to room temperature, the reaction mixture was directly subjected to flash column chromatography (petroleum ether/ethyl acetate $=9: 1$ to $5: 1$ ) to afford the desired product 7 (172 mg, 70\% yield) as a colorless oil. $\mathbf{R}_{\boldsymbol{f}}=0.51$ (petroleum ether/ethyl acetate $\left.=5: 1\right)$. ${ }^{1} \mathbf{H}$ NMR $\left(400 \mathrm{MHz} \mathrm{CDCl}_{3}\right) \delta 7.26(\mathrm{~m}, 5 \mathrm{H}), 7.19(\mathrm{t}, J=7.9 \mathrm{~Hz}, 1 \mathrm{H}), 6.79(\mathrm{t}, J=7.1 \mathrm{~Hz}, 2 \mathrm{H}), 5.96(\mathrm{dt}, J$ $=13.4,6.3 \mathrm{~Hz}, 1 \mathrm{H}), 5.80(\mathrm{~d}, J=10.1 \mathrm{~Hz}, 1 \mathrm{H}), 5.70-5.60(\mathrm{~m}, 1 \mathrm{H}), 5.43-5.32(\mathrm{~m}, 1 \mathrm{H}), 4.55(\mathrm{~d}, J=$ $11.9 \mathrm{~Hz}, 1 \mathrm{H}), 4.49(\mathrm{~d}, J=11.8 \mathrm{~Hz}, 1 \mathrm{H}), 3.86(\mathrm{~m}, 3 \mathrm{H}), 3.80(\mathrm{~s}, 3 \mathrm{H}), 3.61$ (ddd, $J=16.4,9.6,4.3 \mathrm{~Hz}$, $2 \mathrm{H}), 3.53(\mathrm{dd}, J=16.4,6.1 \mathrm{~Hz}, 1 \mathrm{H}), 1.43(\mathrm{~s}, 9 \mathrm{H}) \mathrm{ppm} .{ }^{13} \mathbf{C ~ N M R}\left(150 \mathrm{MHz}, \mathrm{CDCl}_{3}\right) \delta 158.5,155.7$, 139.3, 138.7, 134.6, 128.7, 128.4, 127.8, 127.7, 127.6, 126.0, 123.1, 109.8, 79.2, 72.5, 71.6, 55.5, 48.8, 36.5, 33.3, 28.6 ppm. IR (KBr): 3453, 3086, 3063, 3002, 2917, 1708, 1599, 1497, 1413, 1390, 1364, 1260, 1167, 1021, 926,799, 699, $585 \mathrm{~cm}^{-1}$. HRMS (ESI-TOF) $\mathbf{m} / \mathbf{z}:[\mathrm{M}+\mathrm{Na}]^{+}$Calcd for $\mathrm{C}_{25} \mathrm{H}_{32} \mathrm{BrNO}_{4} \mathrm{Na} 512.1407$; Found 512.1410. $[\alpha]_{\mathrm{D}}^{21.4}=-32.20^{\circ}\left(c=0.36, \mathrm{CHCl}_{3}\right)$. 


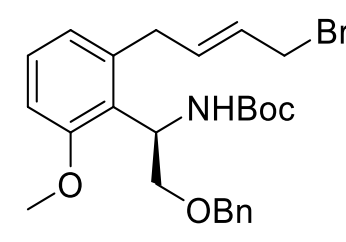

7

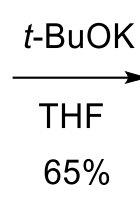

$65 \%$<smiles>C=C[C@@H]1Cc2cccc(OC)c2[C@H](COc2ccccc2)[NH+]1C(=O)O</smiles>

14

Compound 7 (760 mg, $1.56 \mathrm{mmol}, 1.0$ equiv) was dissolved in dry THF (10 mL) and cooled down to $0{ }^{\circ} \mathrm{C}$. Potassium tert-butanolate $\left(260 \mathrm{mg}, 2.3 \mathrm{mmol}, 1.5\right.$ equiv) was added at $0{ }^{\circ} \mathrm{C}$. The reaction mixture was left stirring for $1 \mathrm{~h}$ at room temperature and then quenched with saturated aqueous $\mathrm{NH}_{4} \mathrm{Cl}$ $(10 \mathrm{~mL})$. The mixture was extracted with ethyl acetate $(2 \times 50 \mathrm{~mL})$ and the combined organic phase was washed with brine, dried over anhydrous $\mathrm{Na}_{2} \mathrm{SO}_{4}$. The dried solution was concentrated in vacuo. The crude reaction mixture was directly subjected to flash column chromatography (petroleum ether/ethyl acetate $=9: 1$ to 5:1) to afford the desired product $14(415 \mathrm{mg}, 65 \%$ yield $)$ as a colorless oil . $\mathbf{R}_{\boldsymbol{f}}=0.60$ (petroleum ether/ethyl acetate $\left.=5: 1\right) .{ }^{\mathbf{1}} \mathbf{H} \mathbf{~ N M R}\left(400 \mathrm{MHz}, \mathrm{CDCl}_{3}\right)$ (the major rotamer was listed) $\delta 7.28(\mathrm{~m}, 5 \mathrm{H}), 7.18(\mathrm{t}, J=7.9 \mathrm{~Hz}, 1 \mathrm{H}), 6.74(\mathrm{q}, J=7.6,6.3 \mathrm{~Hz}, 2 \mathrm{H}), 5.98(\mathrm{~s}, 1 \mathrm{H}), 5.21(\mathrm{~d}, J=$ $17.3 \mathrm{~Hz}, 1 \mathrm{H}), 5.05(\mathrm{~d}, J=10.3 \mathrm{~Hz}, 1 \mathrm{H}), 4.88(\mathrm{t}, J=13.8 \mathrm{~Hz}, 1 \mathrm{H}), 4.78-4.59(\mathrm{~m}, 1 \mathrm{H}), 4.53(\mathrm{~d}, J=12.0$ $\mathrm{Hz}, 1 \mathrm{H}), 4.42(\mathrm{q}, J=11.8,9.6 \mathrm{~Hz}, 1 \mathrm{H}), 3.80(\mathrm{~s}, 3 \mathrm{H}), 3.72-3.52(\mathrm{~m}, 2 \mathrm{H}), 2.96(\mathrm{q}, J=15.2 \mathrm{~Hz}, 2 \mathrm{H})$, $1.46(\mathrm{~d}, J=7.3 \mathrm{~Hz}, 9 \mathrm{H}) \mathrm{ppm} .{ }^{13} \mathbf{C} \mathbf{N M R}\left(100 \mathrm{MHz}, \mathrm{CDCl}_{3}\right)$ (the major rotamer was listed) $\delta 155.5$, $142.0,138.9,136.0,128.2,128.1,127.7,127.4,127.3,124.0,119.9,113.6,108.5,79.9,72.5,71.6$, 55.5, 54.6, 49.8, 34.8, 28.6 ppm. IR (KBr): 3082, 2929, 2857, 1688, 1542, 1441, 1389, 1351, 1262, 1169, 1086, 915, 670, $602 \mathrm{~cm}^{-1}$. HRMS (ESI-TOF) $\mathbf{m} / \mathbf{z}$ : $[\mathrm{M}+\mathrm{Na}]^{+}$Calcd for $\mathrm{C}_{25} \mathrm{H}_{31} \mathrm{NO}_{4} \mathrm{Na} 432.2145$; Found 432.2152. $[\alpha]_{\mathrm{D}}{ }^{20.9}=-5.14^{\circ}\left(c=0.74, \mathrm{CHCl}_{3}\right)$.<smiles>C=CC1Cc2cccc(OC)c2C(COc2ccccc2)[N+]1(C)C(=O)O</smiles>

14

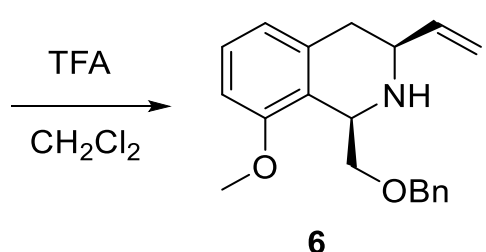

6

$$
\mathrm{Et}_{3} \mathrm{~N}, \mathrm{BOP}-\mathrm{Cl}
$$
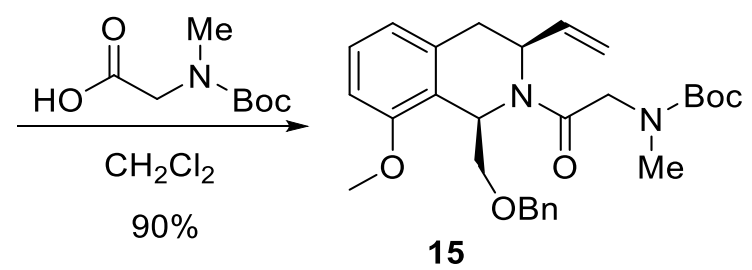

Trifluoroacetic acid (3.7 mL, $50 \mathrm{mmol}, 50$ equiv) was added to an ice-cooled solution of compound 14 (410 mg, $1.0 \mathrm{mmol}, 1.0$ equiv) in dry DCM $(15 \mathrm{~mL})$ and the resulting mixture was stirred for $1 \mathrm{~h}$ at room temperature. The mixture was concentrated under reduced pressure to provide the crude product $\mathbf{6}$. The crude product $\mathbf{6}$ was used in the subsequent step without further purification. The crude product 6 and triethylamine $(0.35 \mathrm{~mL}, 2.5 \mathrm{mmol}, 2.5$ equiv) was dissolved in dry DCM (10 mL) and cooled down to $0{ }^{\circ} \mathrm{C}$. $N$-Boc sarcosine $(208 \mathrm{mg}, 1.1 \mathrm{mmol}, 1.1$ equiv) and BOP-Cl (280 mg, $1.1 \mathrm{mmol}$, 1.1 equiv) was added, and the resulting mixture was stirred at $0{ }^{\circ} \mathrm{C}$ for $12 \mathrm{~h}$. The reaction was quenched with saturated aqueous $\mathrm{NH}_{4} \mathrm{Cl}(5 \mathrm{~mL})$ and extracted with ethyl acetate $(3 \times 10 \mathrm{~mL})$. The combined organic extracts were washed with brine $(5 \mathrm{~mL})$, dried over anhydrous $\mathrm{Na}_{2} \mathrm{SO}_{4}$. The dried solution was concentrated in vacuo. The crude residue was purified by column chromatography (petroleum ether/ethyl acetate $=2: 1$ ) to give compound 15 (434 mg, 90\% yield) as a colorless oil.

Data for compound 6

Compound 6 was isolated for characterization purposes by purifying a small amount of crude by column chromatography (petroleum ether/ethyl acetate $=1: 1$ ). $\mathbf{R}_{\boldsymbol{f}}=0.22$ (petroleum ether/ethyl acetate $=1: 1) .{ }^{1} \mathbf{H}$ NMR $\left(600 \mathrm{MHz}, \mathrm{CDCl}_{3}\right)(15: 1$ mixture of rotamers, the major rotamer was listed) $\delta 7.28$ $(\mathrm{m}, 5 \mathrm{H}), 7.14(\mathrm{t}, J=7.9 \mathrm{~Hz}, 1 \mathrm{H}), 6.73(\mathrm{~d}, J=7.6 \mathrm{~Hz}, 1 \mathrm{H}), 6.69(\mathrm{~d}, J=8.2 \mathrm{~Hz}, 1 \mathrm{H}), 6.01(\mathrm{ddd}, J=$ $16.9,10.4,6.2 \mathrm{~Hz}, 1 \mathrm{H}), 5.29(\mathrm{~d}, J=17.6 \mathrm{~Hz}, 1 \mathrm{H}), 5.16(\mathrm{~d}, J=10.4 \mathrm{~Hz}, 1 \mathrm{H}), 4.58(\mathrm{dd}, J=6.8,2.7 \mathrm{~Hz}$, $1 \mathrm{H}), 4.55-4.45(\mathrm{~m}, 2 \mathrm{H}), 4.12(\mathrm{dd}, J=9.3,2.9 \mathrm{~Hz}, 1 \mathrm{H}), 3.72(\mathrm{~s}, 3 \mathrm{H}), 3.69(\mathrm{dd}, J=9.2,6.6 \mathrm{~Hz}, 1 \mathrm{H})$, $3.42(\mathrm{q}, J=6.7 \mathrm{~Hz}, 1 \mathrm{H}), 2.84(\mathrm{~s}, 1 \mathrm{H}) 2.74(\mathrm{~d}, J=6.9 \mathrm{~Hz}, 2 \mathrm{H}) \mathrm{ppm} .{ }^{13} \mathbf{C} \mathbf{N M R}\left(150 \mathrm{MHz}, \mathrm{CDCl}_{3}\right)$ 
(15:1 mixture of rotamers, the major rotamer was listed) $\delta 156.9,140.3,138.7,138.3,128.4,127.8$, 127.6, 127.3, 123.4, 121.6, 115.5, 108.3, 73.0, 72.9, 55.1, 53.8, 36.8 ppm. IR (KBr):3980, 3647, 3351, 3204, 3084, 3003, 2927, 2506, 1721, 1644, 1586, 1495, 1439, 1361, 1302, 1200, 1092, 994, 821, 779, $560 \mathrm{~cm}^{-1}$. HRMS (ESI-TOF) m/z: $[\mathrm{M}+\mathrm{H}]^{+}$Calcd for $\mathrm{C}_{20} \mathrm{H}_{24} \mathrm{NO}_{2} 310.1802$; Found 310.1802. [a $]_{\mathrm{D}}^{20.9}$ $=-85.62^{\circ}\left(c=0.45, \mathrm{CHCl}_{3}\right)$.

Data for compound 15

$\mathbf{R}_{\boldsymbol{f}}=0.43$ (petroleum ether/ethyl acetate $\left.=2: 1\right) .{ }^{1} \mathbf{H} \mathbf{~ N M R}\left(400 \mathrm{MHz}, \mathrm{CDCl}_{3}\right)$ (the major rotamer was listed) $\delta 7.33-7.27(\mathrm{~m}, 5 \mathrm{H}), 7.19(\mathrm{t}, J=8.0 \mathrm{~Hz}, 1 \mathrm{H}), 6.75(\mathrm{dd}, J=12.1,7.7 \mathrm{~Hz}, 2 \mathrm{H}), 5.95$ (ddd, $J$ $=16.6,10.5,5.2 \mathrm{~Hz}, 1 \mathrm{H}), 5.38(\mathrm{~m}, 1 \mathrm{H}), 5.29-5.11(\mathrm{~m}, 2 \mathrm{H}), 5.05(\mathrm{~d}, J=10.5 \mathrm{~Hz}, 1 \mathrm{H}), 4.64(\mathrm{dd}, J=$ $18.7,13.9 \mathrm{~Hz}, 1 \mathrm{H}), 4.51(\mathrm{dt}, J=19.4,11.5 \mathrm{~Hz}, 2 \mathrm{H}), 4.19(\mathrm{t}, J=16.3 \mathrm{~Hz}, 1 \mathrm{H}), 3.82(\mathrm{~s}, 3 \mathrm{H}), 3.69(\mathrm{dd}$, $J=9.6,4.0 \mathrm{~Hz}, 1 \mathrm{H}), 3.60(\mathrm{t}, J=9.8 \mathrm{~Hz}, 1 \mathrm{H}), 3.13-2.84(\mathrm{~m}, 3 \mathrm{H}), 2.77(\mathrm{~d}, J=25.0 \mathrm{~Hz}, 2 \mathrm{H}), 1.50-1.31$ $(\mathrm{m}, 9 \mathrm{H}) \mathrm{ppm} .{ }^{13} \mathbf{C ~ N M R}\left(125 \mathrm{MHz}, \mathrm{CDCl}_{3}\right)$ (the major rotamer was listed) $\delta 168.9,155.2,140.0,139.6$, $135.3,128.7,128.5,128.5,128.0,127.9,121.9,120.6,114.3,108.3$, 79.5, 73.3, 73.2, 71.0, 55.5, 51.0, 50.8, 47.7, 35.6, 35.5, 31.6, 28.5 ppm. IR (KBr): 3485, 3369, 3063, 3006, 2862, 1736, 1698, 1594, 1476, 1454, 1391, 1304, 1215, 1177, 1100, 972, 786, $699 \mathrm{~cm}^{-1}$. HRMS (ESI-TOF) m/z: [M+Na] $]^{+}$ Calcd for $\mathrm{C}_{28} \mathrm{H}_{36} \mathrm{~N}_{2} \mathrm{O}_{5} \mathrm{Na} 503.2516$; Found 503.2518. $[\alpha]_{\mathrm{D}}{ }^{16.1}=+11.55^{\circ}\left(c=0.16 \mathrm{CHCl}_{3}\right)$.<smiles>C=C[C@@H]1Cc2cccc(OC)c2[C@@H](COc2ccccc2)N1C(=O)CN(C)C(C)(C)C</smiles>

15<smiles>COc1cccc2c1[C@@H](COc1ccccc1)N(C(=O)CN(C)C(=O)OCc1ccccc1)[C@H](C=O)C2</smiles>

5

Compound 15 (520 mg, $1.08 \mathrm{mmol}, 1.0$ equiv) was dissolved in DCM (12 mL) and cooled down to $-78^{\circ} \mathrm{C}$. An $\mathrm{O}_{3}-\mathrm{O}_{2}$ stream was passed through the solution for $15 \mathrm{~min}$. The solution was purged with nitrogen for $15 \mathrm{~min}$, and then $\mathrm{Me}_{2} \mathrm{~S}(0.8 \mathrm{~mL}, 10.8 \mathrm{mmol}, 10.0$ equiv $)$ was added. The reaction mixture was warmed to room temperature and stirred for $8 \mathrm{~h}$. The solution was concentrated in vacuo and the residue was purified by column chromatography (petroleum ether/ethyl acetate $=1: 1$ ) to give compound $\mathbf{5}$ (427 mg, 82\% yield) as a colorless oil. $\mathbf{R}_{\boldsymbol{f}}=0.33$ (petroleum ether/ethyl acetate $\left.=1: 1\right) .{ }^{\mathbf{1}} \mathbf{H}$ NMR $\left(400 \mathrm{MHz}, \mathrm{CDCl}_{3}\right)$ (the major rotamer was listed) $\delta 7.31-7.25(\mathrm{~m}, 5 \mathrm{H}), 7.24(\mathrm{t}, J=8.1 \mathrm{~Hz}, 1 \mathrm{H})$, 6.91- $6.72(\mathrm{~m}, 2 \mathrm{H}), 5.53(\mathrm{td}, J=10.9,10.5,3.4 \mathrm{~Hz}, 1 \mathrm{H}), 4.82-4.61(\mathrm{~m}, 1 \mathrm{H}), 4.65-4.46(\mathrm{~m}, 2 \mathrm{H}), 4.32$ $(\mathrm{dd}, J=16.6,13.8 \mathrm{~Hz}, 1 \mathrm{H}), 4.26-3.97(\mathrm{~m}, 1 \mathrm{H}), 3.83(\mathrm{~d}, J=8.7 \mathrm{~Hz}, 3 \mathrm{H}), 3.74$ (ddd, $J=14.2,9.9,5.1$ $\mathrm{Hz}, 1 \mathrm{H}), 3.46(\mathrm{t}, J=9.8 \mathrm{~Hz}, 1 \mathrm{H}), 3.01-2.94(\mathrm{~m}, 3 \mathrm{H}), 2.83(\mathrm{~d}, J=30.5 \mathrm{~Hz}, 2 \mathrm{H}), 1.52(\mathrm{~d}, J=10.2 \mathrm{~Hz}$, 9H) ppm. ${ }^{13} \mathbf{C}$ NMR $\left(100 \mathrm{MHz}, \mathrm{CDCl}_{3}\right.$ ) (the major rotamer was listed) $\delta 200.0,170.5,165.4,156.6$, 155.1, 137.5, 134.0, 129.1, 128.5, 128.0, 121.4, 120.6, 108.7, 79.8, 73.5, 71.6, 59.1, 55.6, 50.4, 50.1, 35.5, 28.2, 26.4 ppm. IR (KBr): 3432, 3366, 3087, 3003, 2927, 2721, 1734, 1697, 1543, 1454, 1366, 1304, 1261, 1100, 1063, 972, 800, $666 \mathrm{~cm}^{-1}$. HRMS (ESI-TOF) $\mathbf{m} / \mathbf{z}:[\mathrm{M}+\mathrm{Na}]^{+}$Calcd for $\mathrm{C}_{27} \mathrm{H}_{34} \mathrm{~N}_{2} \mathrm{O}_{6} \mathrm{Na}$ 505.2309; Found 505.2317. Optical rotation: $[\boldsymbol{\alpha}]_{\mathrm{D}}{ }^{19.1}=-9.01^{\circ}\left(c=0.45 \mathrm{CHCl}_{3}\right)$.<smiles>COc1cccc2c1[C@H](COCc1ccccc1)N(C(=O)CN(C)C(C)(C)C)C2C=O</smiles>

5

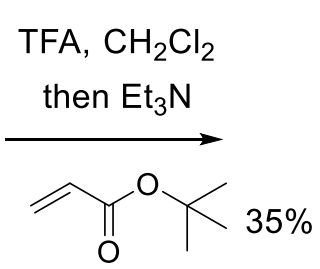<smiles>COc1cccc2c1[C@@H](COBr)N1C(=O)[C@H]3[C@H](COC(C)(C)C)C[C@H]([C@H]21)N3C</smiles> 
Trifluoroacetic acid (1.6 mL, $142 \mathrm{mmol}, 50$ equiv) was added to a solution of compound 5 (210 $\mathrm{mg}, 0.44 \mathrm{mmol}, 1.0$ equiv) in DCM $(22 \mathrm{~mL}, 0.02 \mathrm{M})$ and the resulting mixture was stirred for $2 \mathrm{~h}$ at room temperature. The solution was concentrated in vacuo. The residue was dissolved in dry DCM $(22 \mathrm{~mL})$ and cooled to $0{ }^{\circ} \mathrm{C}$, then tert-butyl acrylate $(1.3 \mathrm{~mL}, 8.8 \mathrm{mmol}, 20$ equiv) and triethylamine ( $0.6 \mathrm{~mL}, 4.4 \mathrm{mmol}, 1.0$ equiv) were added successively. The resulting mixture was warmed to room temperature and was stirred at that temperature for $8 \mathrm{~h}$. The mixture was concentrated under reduced pressure. The crude residue was purified by column chromatography (petroleum ether/ethyl acetate = 3:1) to give compound $\mathbf{1 6}\left(75 \mathrm{mg}, 35 \%\right.$ yield) as a yellow foam. $\mathbf{R}_{\boldsymbol{f}}=0.53$ (petroleum ether/ethyl acetate $=3: 1) .{ }^{1} \mathbf{H}$ NMR $\left(400 \mathrm{MHz}, \mathrm{CDCl}_{3}\right) \delta 7.34-7.20(\mathrm{~m}, 6 \mathrm{H}), 6.73(\mathrm{dd}, J=19.0,7.9 \mathrm{~Hz}, 2 \mathrm{H}), 6.42$ $(\mathrm{dd}, J=7.3,3.6 \mathrm{~Hz}, 1 \mathrm{H}), 5.71(\mathrm{~s}, 1 \mathrm{H}), 4.66(\mathrm{~d}, J=12.1 \mathrm{~Hz}, 1 \mathrm{H}), 4.44(\mathrm{~d}, J=12.1 \mathrm{~Hz}, 1 \mathrm{H}), 4.22(\mathrm{~s}, 1 \mathrm{H})$, $3.83(\mathrm{~s}, 3 \mathrm{H}), 3.80(\mathrm{~d}, J=7.4 \mathrm{~Hz}, 1 \mathrm{H}), 3.69(\mathrm{dd}, J=10.8,7.3 \mathrm{~Hz}, 1 \mathrm{H}), 3.56(\mathrm{dd}, J=10.7,3.6 \mathrm{~Hz}, 1 \mathrm{H})$, $2.82(\mathrm{dd}, J=9.9,5.0 \mathrm{~Hz}, 1 \mathrm{H}), 2.58(\mathrm{~s}, 3 \mathrm{H}), 2.57(\mathrm{~m}, 1 \mathrm{H}), 2.19(\mathrm{dd}, J=13.4,9.9 \mathrm{~Hz}, 1 \mathrm{H}), 1.52(\mathrm{~s}, 9 \mathrm{H})$ ppm. ${ }^{13} \mathbf{C}$ NMR $\left(100 \mathrm{MHz}, \mathrm{CDCl}_{3}\right) \delta 172.3,170.7,155.4,138.4,137.1,133.0,128.9,128.2,127.5$, 127.3, 117.1, 116.8, 109.0, 103.9, 81.4, 72.4, 70.0, 66.1, 65.0, 55.4, 51.4, 47.2, 35.0, 32.6, $28.2 \mathrm{ppm}$. IR (KBr): 3438, 3366, 3160, 3028, 2929, 2323, 1728, 1639, 1600, 1576, 1474, 1441, 1404, 1366, 1324, 1243, 1154, 1100, 954, 847, 740, 663, $549 \mathrm{~cm}^{-1}$. HRMS (ESI-TOF) $\mathbf{m} / \mathbf{z}:[\mathrm{M}+\mathrm{H}]^{+}$Calcd for $\mathrm{C}_{29} \mathrm{H}_{35} \mathrm{~N}_{2} \mathrm{O}_{5} 491.2540$; Found 491.2539. $[\alpha]_{\mathrm{D}}{ }^{18.9}=+241.84^{\circ}\left(c=0.32 \mathrm{CHCl}_{3}\right)$.
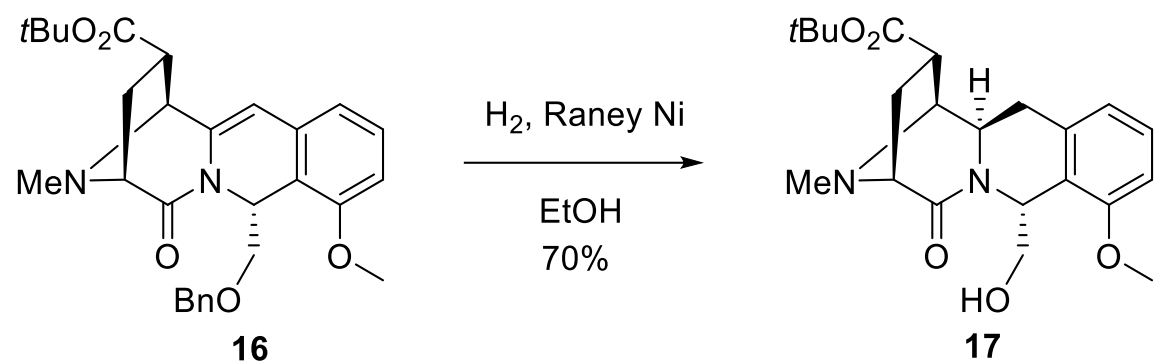

Compound 16 (50 mg, $0.1 \mathrm{mmol})$ was dissolved in absolute EtOH $(4 \mathrm{~mL})$ in a pressure vessel, and Raney Nickel $(0.4 \mathrm{~mL})$ was added. The vessel was pressurized to $100 \mathrm{psi}$ with hydrogen and then stirred under hydrogen for a further $24 \mathrm{~h}$. The reaction mixture was flushed with argon and was filtered through a Celite pad washing with ethyl acetate. The filtrate was concentrated in vacuo and the residue was purified by flash chromatography $\left(\mathrm{MeOH} / \mathrm{CH}_{2} \mathrm{Cl}_{2}=1: 15\right)$ to provide compound 17 (28 mg, 70\% yield) as a white foam. $\mathbf{R}_{\boldsymbol{f}}=0.46\left(\mathrm{MeOH} / \mathrm{CH}_{2} \mathrm{Cl}_{2}=1: 10\right) .{ }^{1} \mathbf{H} \mathbf{N M R}\left(600 \mathrm{MHz}, \mathrm{CDCl}_{3}\right) \delta 7.19(\mathrm{t}, J=$ $7.9 \mathrm{~Hz}, 1 \mathrm{H}), 6.77(\mathrm{~d}, J=7.7 \mathrm{~Hz}, 1 \mathrm{H}), 6.72(\mathrm{~d}, J=8.2 \mathrm{~Hz}, 1 \mathrm{H}), 5.89(\mathrm{dd}, J=7.9,3.4 \mathrm{~Hz}, 1 \mathrm{H}), 4.49$ (ddd, $J=10.4,5.7,3.7 \mathrm{~Hz}, 1 \mathrm{H}), 4.00(\mathrm{dd}, J=11.6,3.5 \mathrm{~Hz}, 1 \mathrm{H}), 3.83(\mathrm{~s}, 3 \mathrm{H}), 3.79$ (dd, $J=11.5,7.8$ $\mathrm{Hz}, 1 \mathrm{H}), 3.64(\mathrm{t}, J=5.4 \mathrm{~Hz}, 2 \mathrm{H}), 3.08(\mathrm{dd}, J=9.5,6.5 \mathrm{~Hz}, 1 \mathrm{H}), 2.88(\mathrm{qd}, J=17.1,8.3 \mathrm{~Hz}, 2 \mathrm{H}), 2.58$ $(\mathrm{s}, 3 \mathrm{H}), 2.56-2.47(\mathrm{~m}, 1 \mathrm{H}), 2.35(\mathrm{br}, 1 \mathrm{H}) 2.13(\mathrm{dd}, J=13.2,9.6 \mathrm{~Hz}, 1 \mathrm{H}), 1.48(\mathrm{~s}, 9 \mathrm{H}) \mathrm{ppm} .{ }^{13} \mathbf{C}$ NMR $\left(150 \mathrm{MHz}, \mathrm{CDCl}_{3}\right) \delta 173.5,172.8,156.2,133.7,128.2,121.0,120.9,108.1,81.3,66.4,66.0,64.6$, 55.5, 49.5, 49.1, 42.6, 36.7, 32.5, 29.9, 28.2 ppm. IR (KBr): 3369, 2963, 2919, 2849, 1724, 1604, 1539, 1470, 1392, 1324, 1224, 1152, 1036, 1019, 959,869, 757, $631 \mathrm{~cm}^{-1}$. HRMS (ESI-TOF) m/z: $[\mathrm{M}+\mathrm{H}]^{+}$Calcd for $\mathrm{C}_{22} \mathrm{H}_{31} \mathrm{~N}_{2} \mathrm{O}_{5} 403.2227$; Found 403.2231. $[\alpha]_{\mathrm{D}}{ }^{18.1}=+60.15^{\circ}\left(c=0.20, \mathrm{CHCl}_{3}\right)$.<smiles>COC(=O)C1C[C@H]2[C@@H](C)c3cccc(OC)c3[C@@H](CO)N3C(=O)[C@H]2[C@@H]13</smiles>

17

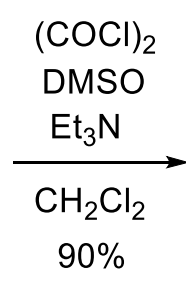

To a solution of oxalyl chloride ( $34 \mu \mathrm{L}, 0.4 \mathrm{mmol}, 2.0$ equiv) in DCM ( $3 \mathrm{~mL})$ at $-78{ }^{\circ} \mathrm{C}$ was added DMSO $\left(57 \mu \mathrm{L}, 0.8 \mathrm{mmol}, 4.0\right.$ equiv). The solution was stirred at $-78{ }^{\circ} \mathrm{C}$ for $10 \mathrm{~min}$. A solution of 
compound 17 ( $81 \mathrm{mg}, 0.2 \mathrm{mmol}, 1.0$ equiv) in $1 \mathrm{~mL}$ of DCM was then added to the reaction mixture dropwise. The resulting solution was stirred at $-78^{\circ} \mathrm{C}$ and after one hour was treated with triethylamine ( $280 \mu \mathrm{L}, 2.0 \mathrm{mmol}, 10.0$ equiv). The reaction mixture was allowed to warm to room temperature. The reaction was quenched with saturated aqueous $\mathrm{NaHCO}_{3}(5 \mathrm{~mL})$ and extracted with $\mathrm{DCM}(3 \times 10 \mathrm{~mL})$. The combined organic extracts were washed with brine $(5 \mathrm{~mL})$, dried over anhydrous $\mathrm{Na}_{2} \mathrm{SO}_{4}$. The dried solution was concentrated in vacuo. The crude residue was purified by column chromatography (petroleum ether/ethyl acetate $=1: 1)$ to afford $18\left(71 \mathrm{mg}, 90 \%\right.$ yield) as a colorless oil. $\mathbf{R}_{f}=0.52$ (petroleum ether/ethyl acetate = 1:1). ${ }^{1} \mathbf{H}$ NMR $\left(600 \mathrm{MHz}, \mathrm{CDCl}_{3}\right) \delta 9.73(\mathrm{~s}, 1 \mathrm{H}), 7.26(\mathrm{~m}, 1 \mathrm{H}), 6.95-$ $6.65(\mathrm{~m}, 2 \mathrm{H}), 6.28(\mathrm{~s}, 1 \mathrm{H}), 4.20(\mathrm{dt}, J=11.6,4.1 \mathrm{~Hz}, 1 \mathrm{H}), 3.90(\mathrm{~s}, 3 \mathrm{H}), 3.71(\mathrm{~d}, J=5.7 \mathrm{~Hz}, 2 \mathrm{H}), 3.14$ $(\mathrm{dd}, J=9.7,6.4 \mathrm{~Hz}, 1 \mathrm{H}), 2.92(\mathrm{dd}, J=16.9,11.6 \mathrm{~Hz}, 1 \mathrm{H}), 2.79(\mathrm{~s}, 3 \mathrm{H}), 2.75(\mathrm{~d}, J=4.8 \mathrm{~Hz}, 1 \mathrm{H}), 2.68$ $2.48(\mathrm{~m}, 1 \mathrm{H}), 2.18(\mathrm{dd}, J=13.3,9.7 \mathrm{~Hz}, 1 \mathrm{H}), 1.49(\mathrm{~s}, 9 \mathrm{H}) \mathrm{ppm} .{ }^{13} \mathbf{C} \mathbf{N M R}\left(150 \mathrm{MHz}, \mathrm{CDCl}_{3}\right) \delta 196.1$, 173.0, 171.9, 156.0, 133.7, 129.0, 121.5, 116.3, 108.5, 81.6, 77.4, 65.9, 65.4, 57.6, 55.7, 50.6, 42.8, 36.1, 33.2, 29.9, 28.2 ppm. IR (KBr): 3430, 3359, 2962, 2919, 2724, 1663, 1472, 1439, 1392, 1368, 1301, 1153, 1097, 1024, 926, 845, 708, $572 \mathrm{~cm}^{-1}$. HRMS (ESI-TOF) m/z: $[\mathrm{M}+\mathrm{H}]^{+}$Calcd for

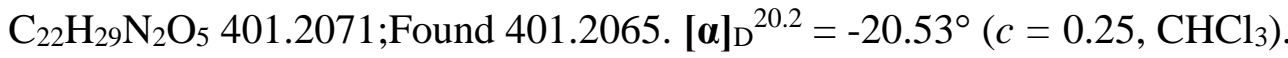<smiles>COc1cccc2c1[C@@H](C=O)N1C[C@@H]2[C@H]2C[C@@H](COC(C)(C)C)[C@H](C1=O)N2C</smiles>

18

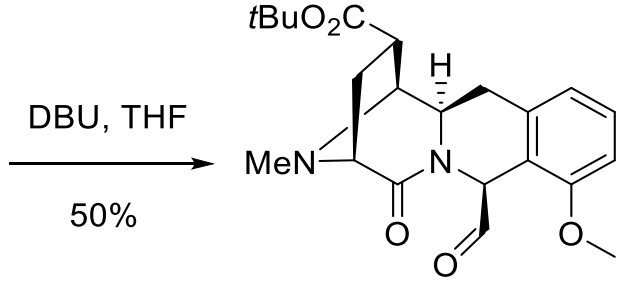

19

To a stirred solution of compound 18 (70 mg, $0.175 \mathrm{mmol}, 1.0$ equiv) in THF ( $5 \mathrm{~mL}$ ) was added DBU $(30 \mu \mathrm{L}, 0.175 \mathrm{mmol}, 1.0$ equiv). The solution was allowed to stir at room temperature for $24 \mathrm{~h}$. Saturated aqueous $\mathrm{NaHCO}_{3}$ was added and the aqueous layer was extracted with DCM $(3 \times 5 \mathrm{~mL})$. The combined organic layers were dried over anhydrous $\mathrm{Na}_{2} \mathrm{SO}_{4}$. The dried solution was concentrated in vacuo. The crude product was purified by flash chromatography (petroleum ether/ethyl acetate $=$ $2: 1$ to $1: 1)$ to afford 19 (35 mg, 50\% yield) as a colorless oil along with unreacted aldehyde 18 (17 mg, $25 \%$ yield). $\mathbf{R}_{\boldsymbol{f}}=0.31$ (petroleum ether/ethyl acetate $\left.=1: 1\right) .{ }^{1} \mathbf{H} \mathbf{~ N M R}\left(600 \mathrm{MHz}, \mathrm{CDCl}_{3}\right) \delta 9.51(\mathrm{~s}$, $1 \mathrm{H}), 7.26(\mathrm{~m}, 1 \mathrm{H}), 6.85(\mathrm{~d}, J=8.4 \mathrm{~Hz}, 1 \mathrm{H}), 6.80(\mathrm{~d}, J=7.7 \mathrm{~Hz}, 1 \mathrm{H}), 6.20(\mathrm{~s}, 1 \mathrm{H}), 3.93(\mathrm{~d}, J=12.0$ $\mathrm{Hz}, 1 \mathrm{H}), 3.90(\mathrm{~s}, 3 \mathrm{H}), 3.72(\mathrm{~s}, 1 \mathrm{H}), 3.66(\mathrm{~d}, J=6.5 \mathrm{~Hz}, 1 \mathrm{H}), 3.44(\mathrm{dd}, J=9.8,6.7 \mathrm{~Hz}, 1 \mathrm{H}), 2.87$ - 2.67 (m, 1H), $2.68-2.57(\mathrm{~m}, 2 \mathrm{H}), 2.51(\mathrm{~s}, 3 \mathrm{H}), 2.44(\mathrm{dd}, J=13.2,9.7 \mathrm{~Hz}, 1 \mathrm{H}), 1.50(\mathrm{~s}, 9 \mathrm{H}) \mathrm{ppm} .{ }^{13} \mathbf{C}$ NMR $\left(150 \mathrm{MHz}, \mathrm{CDCl}_{3}\right) \delta 193.8,173.5,170.1,156.4,137.4,129.7,120.7,115.4,109.3,81.4,66.7,66.5$, 58.0, 55.8, 54.7, 42.4, 37.7, 33.9, 31.7, 28.2 ppm. IR (KBr): 3423, 2963, 2849, 1726, 1602, 1475, 1475, 1393, 1322, 1151, 1301, 1094, 1022, 961, 844, 703, 661, $568 \mathrm{~cm}^{-1}$. HRMS (ESI-TOF) m/z: $[\mathrm{M}+\mathrm{Na}]^{+} \mathrm{Calcd}$ for $\mathrm{C}_{22} \mathrm{H}_{28} \mathrm{~N}_{2} \mathrm{O}_{5} \mathrm{Na} 423.1892$; Found 423.1890. $[\boldsymbol{\alpha}]_{\mathrm{D}}{ }^{18.9}=+303.07^{\circ}\left(c=0.24, \mathrm{CHCl}_{3}\right)$.

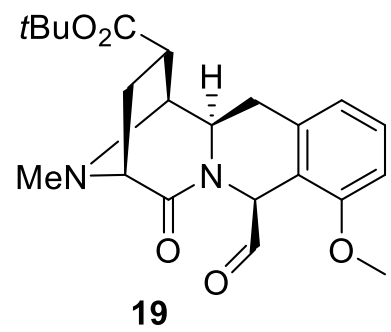

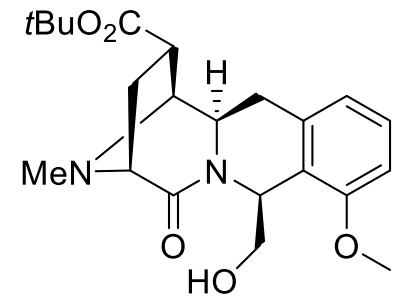

20

To a stirred solution of compound $19(30 \mathrm{mg}, 0.076 \mathrm{mmol}, 1.0$ equiv) in absolute EtOH ( $1 \mathrm{~mL})$ at $0{ }^{\circ} \mathrm{C}$ was added sodium borohydride $\left(12 \mathrm{mg}, 0.3 \mathrm{mmol}, 4\right.$ equiv). This mixture was stirred at $0{ }^{\circ} \mathrm{C}$ for $1 \mathrm{~h}$. Aqueous $1 \mathrm{M} \mathrm{HCl}$ was added dropwise until there was no more $\mathrm{H}_{2}$ evolution. Saturated aqueous 
$\mathrm{NaHCO}_{3}$ was added and the aqueous layer was extracted with ethyl acetate $(3 \times 5 \mathrm{~mL})$ and the combined organic layers were washed with brine, dried over anhydrous $\mathrm{Na}_{2} \mathrm{SO}_{4}$. The dried solution was concentrated in vacuo. The crude product was purified via flash chromatography (petroleum ether/ethyl acetate $=1: 2)$ to afford $26 \mathrm{mg} \mathbf{2 0}\left(24 \mathrm{mg}, 80 \%\right.$ yield) as a white foam. $\mathbf{R}_{\boldsymbol{f}}=0.25$ (petroleum ether/ethyl acetate $=1: 1) .{ }^{1} \mathbf{H}$ NMR $\left(400 \mathrm{MHz}, \mathrm{CDCl}_{3}\right) \delta 7.21(\mathrm{t}, J=7.9 \mathrm{~Hz}, 1 \mathrm{H}), 6.79(\mathrm{dd}, J=13.2$, $7.9 \mathrm{~Hz}, 2 \mathrm{H}), 5.72(\mathrm{dd}, J=5.8,3.0 \mathrm{~Hz}, 1 \mathrm{H}), 3.95-3.87(\mathrm{~m}, 1 \mathrm{H}), 3.84(\mathrm{~s}, 3 \mathrm{H}), 3.71-3.64(\mathrm{~m}, 3 \mathrm{H}), 3.56$ $(\mathrm{dd}, J=11.2,5.8 \mathrm{~Hz}, 1 \mathrm{H}), 3.23(\mathrm{t}, J=8.2 \mathrm{~Hz}, 1 \mathrm{H}), 2.96(\mathrm{t}, J=13.4 \mathrm{~Hz}, 1 \mathrm{H}), 2.68-2.56(\mathrm{~m}, 2 \mathrm{H}), 2.50$ $(\mathrm{s}, 3 \mathrm{H}), 2.44-2.33(\mathrm{~m}, 1 \mathrm{H}), 1.49(\mathrm{~s}, 9 \mathrm{H}) \mathrm{ppm} .{ }^{13} \mathbf{C}$ NMR $\left(150 \mathrm{MHz}, \mathrm{CDCl}_{3}\right) \delta 173.3,173.1,156.0$, 137.2, 128.5, 121.6, 120.1, 109.3, 81.6, 67.5, 67.4, 66.7, 55.8, 55.6, 52.0, 42.4, 37.7, 34.2, 32.0, 28.2 ppm. IR (KBr): 3392, 2963, 2852, 1723, 1605, 1474, 1414, 1325, 1260, 1092, 919, 866, 799, 662,

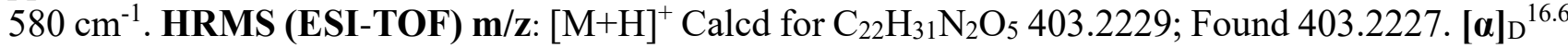
$=+102.52^{\circ}\left(c=0.18, \mathrm{CHCl}_{3}\right)$.<smiles>COC(=O)C1C[C@H]2[C@@H](C)c3cccc(OC)c3[C@@H](CO)N3C(=O)[C@H]2[C@@H]13</smiles>

20

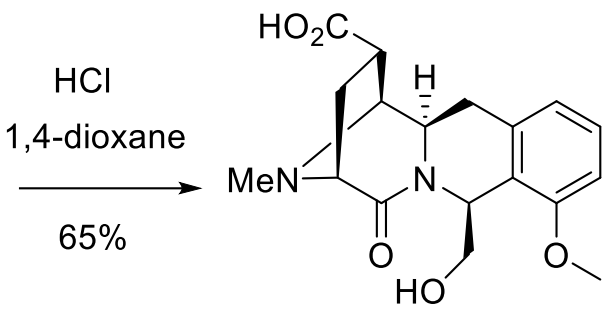

$(+)-3$

To a solution of compound $\mathbf{2 0}$ (20 mg, $0.05 \mathrm{mmol}, 1.0$ equiv) in 1,4-Dioxane ( $0.3 \mathrm{~mL})$ was added HCl-1,4-Dioxane (190 $\mu \mathrm{L}, 4 \mathrm{M}$ in 1,4-Dioxane, 12.0 equiv). The solution was allowed to stir at room temperature for $4 \mathrm{~h}$ and was concentrated under reduced pressure. To a stirred solution of residue in $\mathrm{MeOH}(1 \mathrm{~mL})$ was added ammonia water $\left(\mathrm{NH}_{3} \cdot \mathrm{H}_{2} \mathrm{O}, 5 \mathrm{M}, 10 \mu \mathrm{L}\right)$ at $0{ }^{\circ} \mathrm{C}$ for $5 \mathrm{~min}$. The mixture was concentrated under vacuum, and the residue was purified by flash reverse-phase chromatography on $\mathrm{C} 18$ column (methanol/water $=1: 1)$ to give pure $(+)$-quinocarcinamide $3,(11 \mathrm{mg}, 65 \%$ yield $)$ as white foam. ${ }^{1} \mathbf{H}$ NMR $\left(600 \mathrm{MHz}, \mathrm{D}_{2} \mathrm{O}\right) \delta 7.32(\mathrm{t}, J=7.9 \mathrm{~Hz}, 1 \mathrm{H}), 7.02(\mathrm{~d}, J=7.9 \mathrm{~Hz}, 1 \mathrm{H}), 6.93(\mathrm{~d}, J=7.9$ $\mathrm{Hz}, 1 \mathrm{H}), 5.44(\mathrm{t}, J=3.6 \mathrm{~Hz}, 1 \mathrm{H}), 3.92(\mathrm{dd}, J=11.5,4.3 \mathrm{~Hz}, 1 \mathrm{H}), 3.85(\mathrm{~s}, 3 \mathrm{H}), 3.84-3.72(\mathrm{~m}, 2 \mathrm{H})$, $3.71-3.61(\mathrm{dd}, J=11.5,3.0 \mathrm{~Hz}, 1 \mathrm{H}), 3.58(\mathrm{~d}, J=6.4 \mathrm{~Hz}, 1 \mathrm{H}), 3.21(\mathrm{dd}, J=9.7,6.6 \mathrm{~Hz}, 1 \mathrm{H}), 3.01$ (dd, $J=14.8,12.3 \mathrm{~Hz}, 1 \mathrm{H}), 2.77(\mathrm{dd}, J=14.8,2.4 \mathrm{~Hz}, 1 \mathrm{H}), 2.58$ (ddd, $J=13.3,6.6,6.6 \mathrm{~Hz}, 1 \mathrm{H}), 2.37$ (s, $3 \mathrm{H}), 2.32(\mathrm{dd}, J=13.3,9.8 \mathrm{~Hz}, 1 \mathrm{H}) \mathrm{ppm} .{ }^{13} \mathbf{C}$ NMR $\left(150 \mathrm{MHz}, \mathrm{D}_{2} \mathrm{O}\right) \delta 173.1,171.0,155.8,138.3$, 128.6, 121.2, 120.2, 109.7, 66.8, 66.3, 62.4, 56.2, 55.6, 51.2, 43.4, 36.8, 34.2, 31.1 ppm. IR (KBr): 3975, 3942, 3888, 3820, 3764, 3670, 3305, 2927, 2852, 2536, 2496, 2322, 2222, 2141, 2036, 2002, 1931, 1730, 1589, 1166, 947, 870, 741, 661, $570 \mathrm{~cm}^{-1}$. HRMS (ESI-TOF) m/z: $[\mathrm{M}+\mathrm{H}]^{+}$Calcd for $\mathrm{C}_{18} \mathrm{H}_{23} \mathrm{~N}_{2} \mathrm{O}_{5}$ 347.1601; Found 347.1602. $[\alpha]_{\mathrm{D}}^{23.2}=+44.48^{\circ}(c=0.51, \mathrm{MeOH})$.

The analytical data were consistent with those of natural (-)-quinocarcinamide. ${ }^{2}$

\section{References}

[1] Difieber, C.; Ariger, M. A.; Moriel, P.; Carreira, E. M. Angew. Chem. Int. Ed., 2007, 46, 3139.

[2] (a) Williams, R. W.; Glinka, T.; Flanagan, M. E.; Gallegos, R.; Coffman, H.; Pei, D. J. Am. Chem. Soc. 1992, 114, 733. (b) Flanagan, M. E.; Williams, R. M. J. Org. Chem. 1995, 60, 6791. (c) Wu Y C, Liron M, Zhu J. J. Am. Chem. Soc. 2008, 130, 7148. 


\section{NMR Spectra}
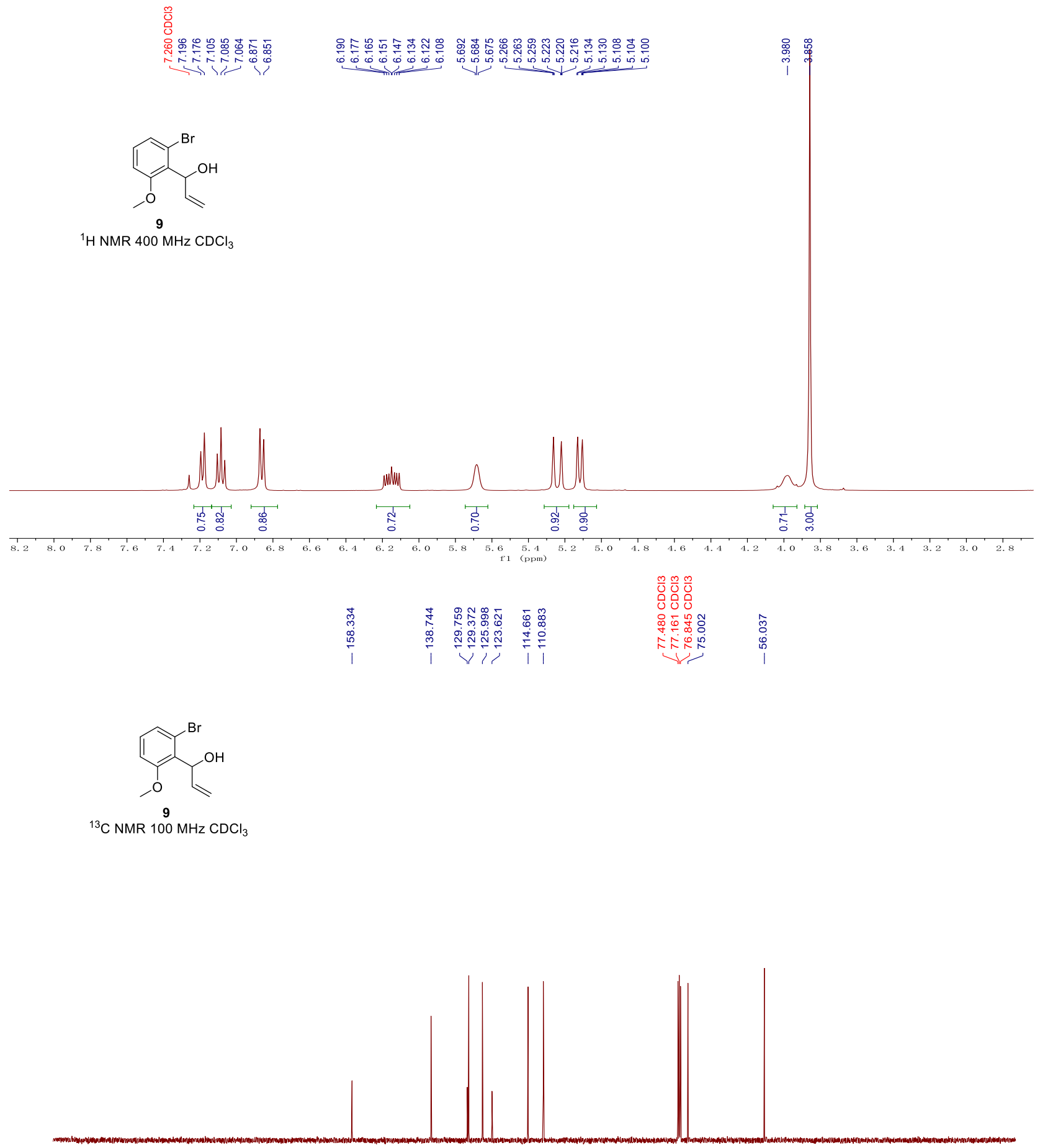

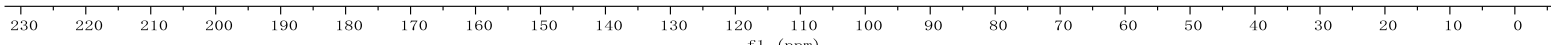




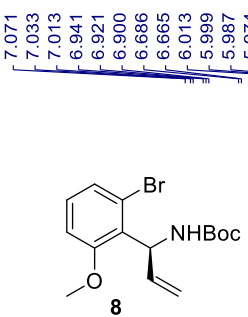

${ }^{1} \mathrm{H}$ NMR $400 \mathrm{MHz} \mathrm{CDCl}_{3}$
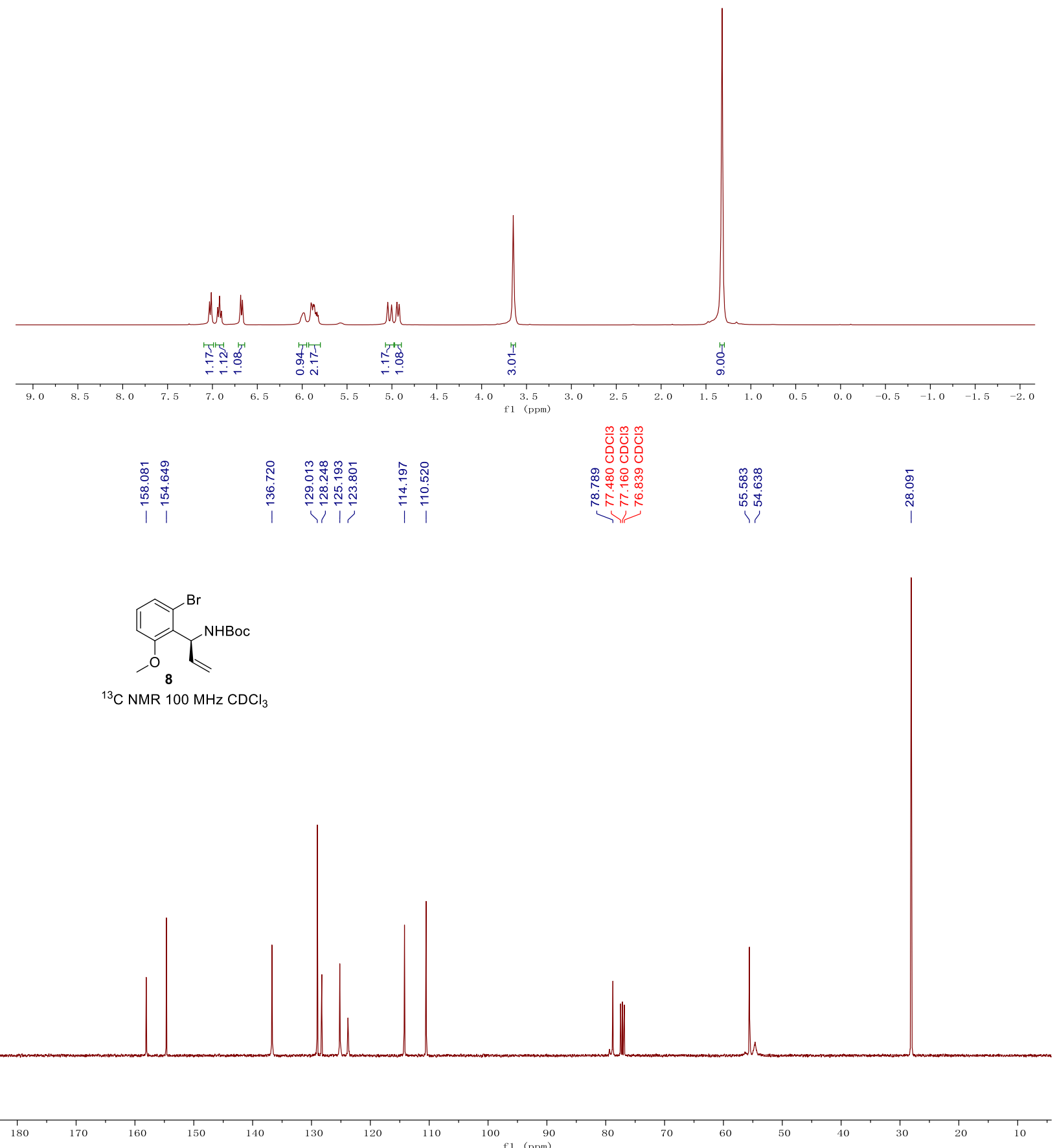
잉

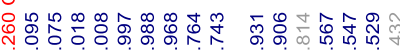

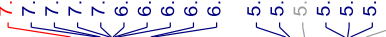

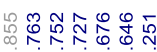

minmim

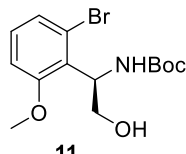

11

${ }^{1} \mathrm{H}$ NMR $400 \mathrm{MHz} \mathrm{CDCl}_{3}$

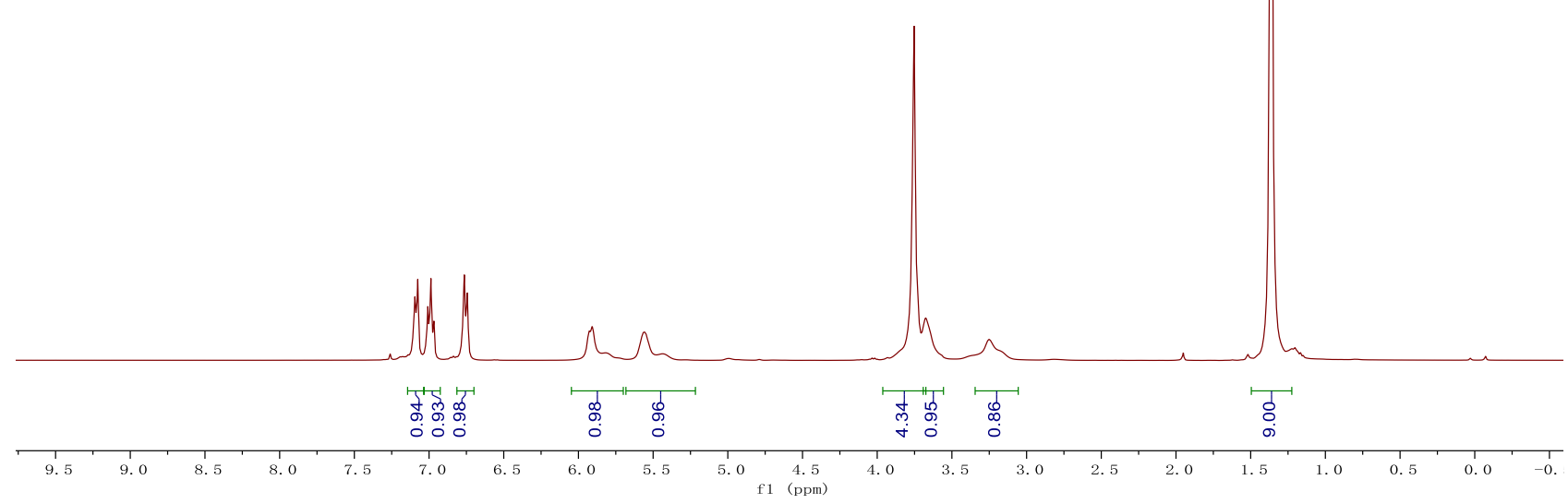

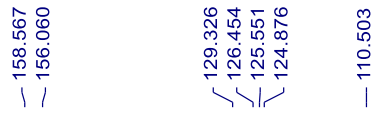

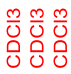

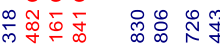

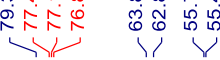

$\underset{\substack{+\stackrel{\infty}{\infty}}}{\stackrel{\infty}{N}}$

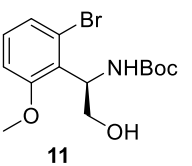

${ }^{13} \mathrm{C} \mathrm{NMR} 100 \mathrm{MHz} \mathrm{CDCl}_{3}$

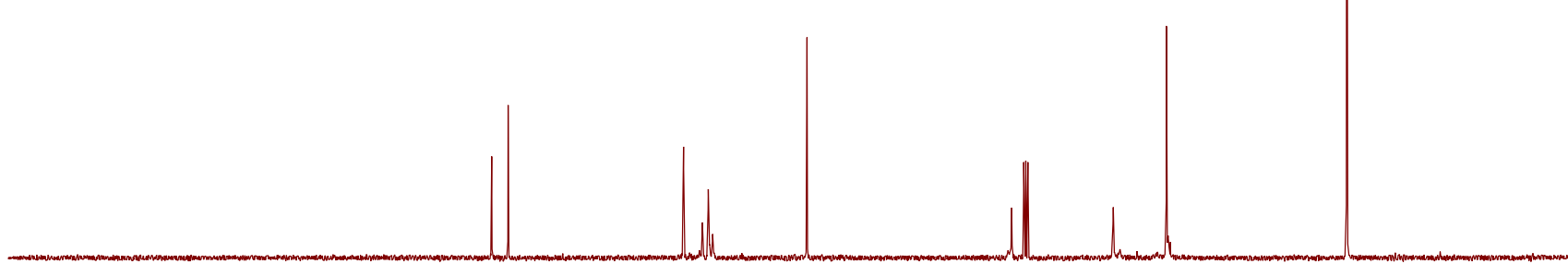

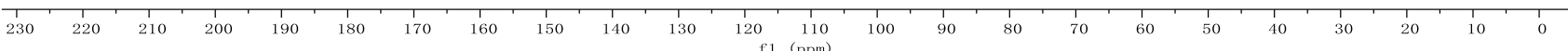




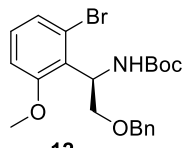

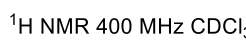

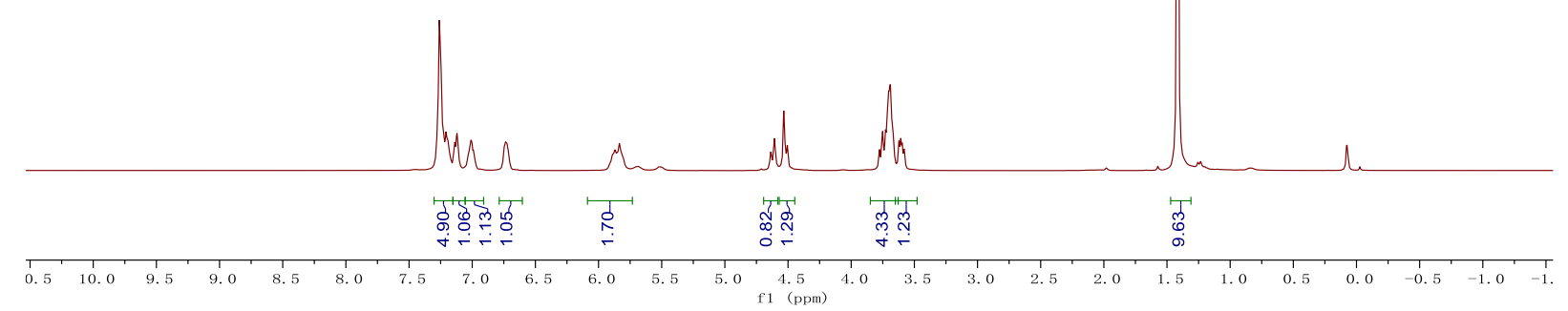

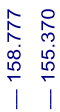

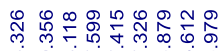

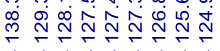

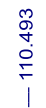

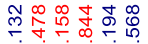

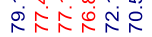

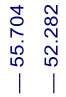

$\underset{\substack{\infty \\ \infty}}{\infty}$

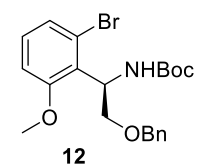

${ }^{13} \mathrm{C}$ NMR $100 \mathrm{MHz} \mathrm{CDCl}_{3}$

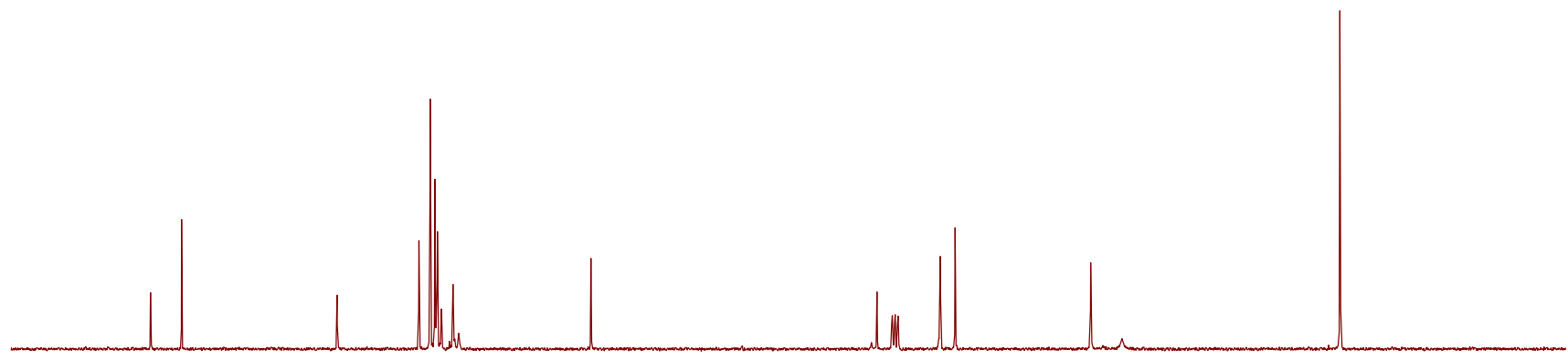

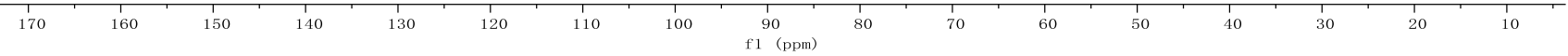




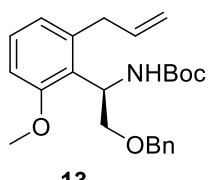

${ }^{1} \mathrm{H}$ NMR $600 \mathrm{MHz} \mathrm{D}_{6}$-acetone

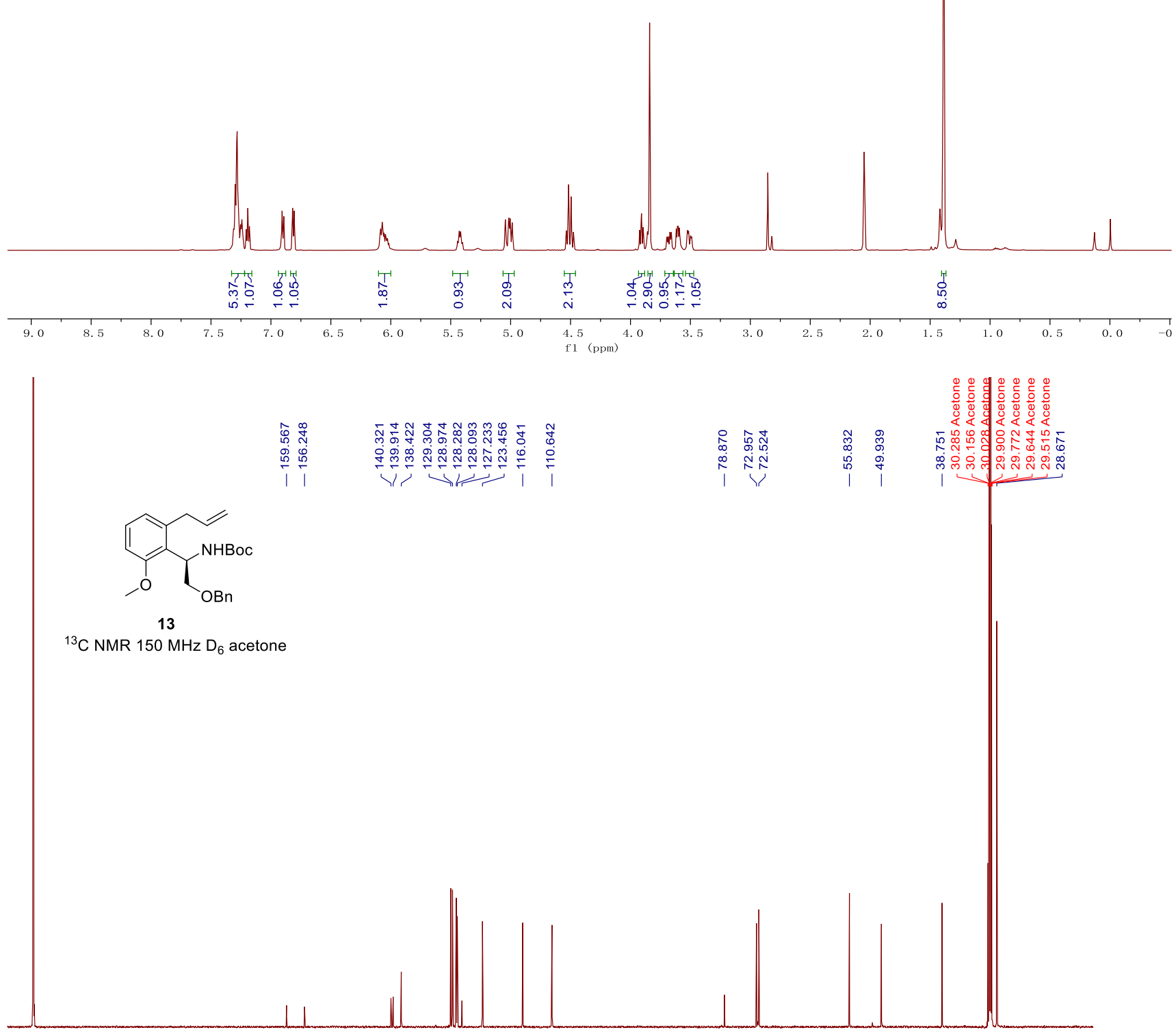

$\underset{210}{T}$

$200 \quad 190 \quad \frac{1}{180} \quad 170 \quad 160$ $120 \quad \frac{110}{(10}$ 


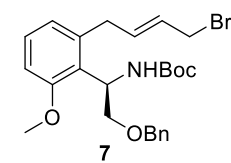

${ }^{1} \mathrm{H} \mathrm{NMR} 400 \mathrm{MHz} \mathrm{CDCl}_{3}$

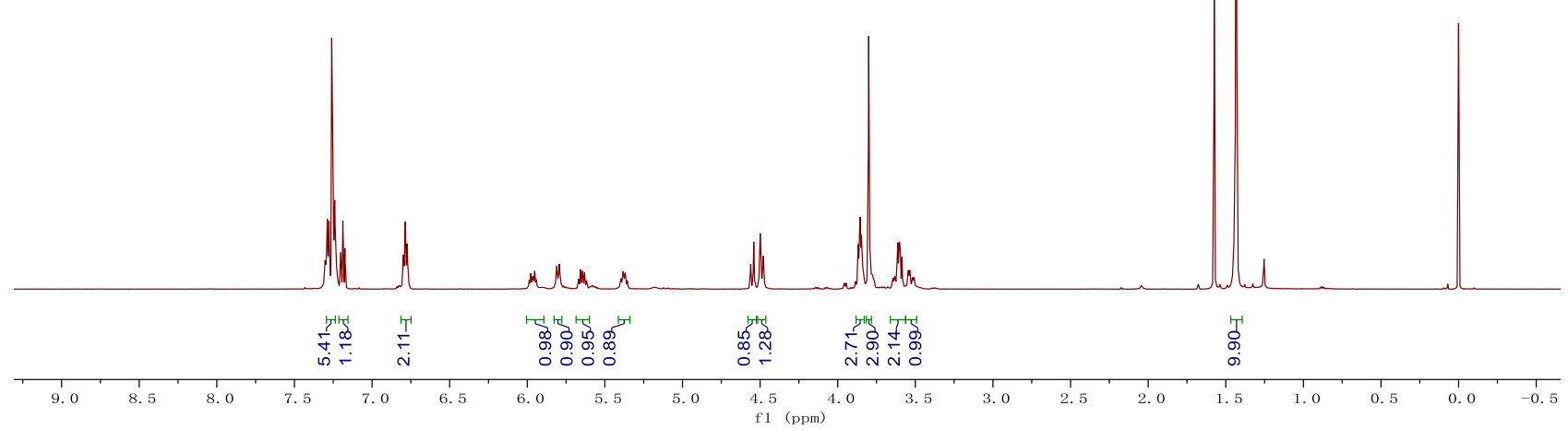

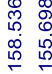

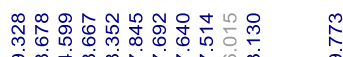

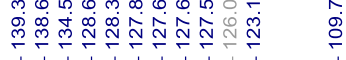

\section{$\begin{array}{ll}\frac{m}{0} & \frac{m}{0} \\ 0 & 0 \\ 0 & 0 \\ 0\end{array}$} ।

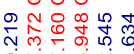

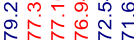

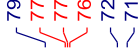

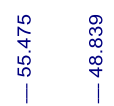

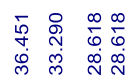

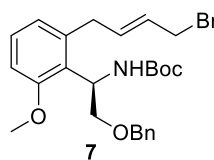

${ }^{13} \mathrm{C} \mathrm{NMR} 150 \mathrm{MHz} \mathrm{CDCl}_{3}$
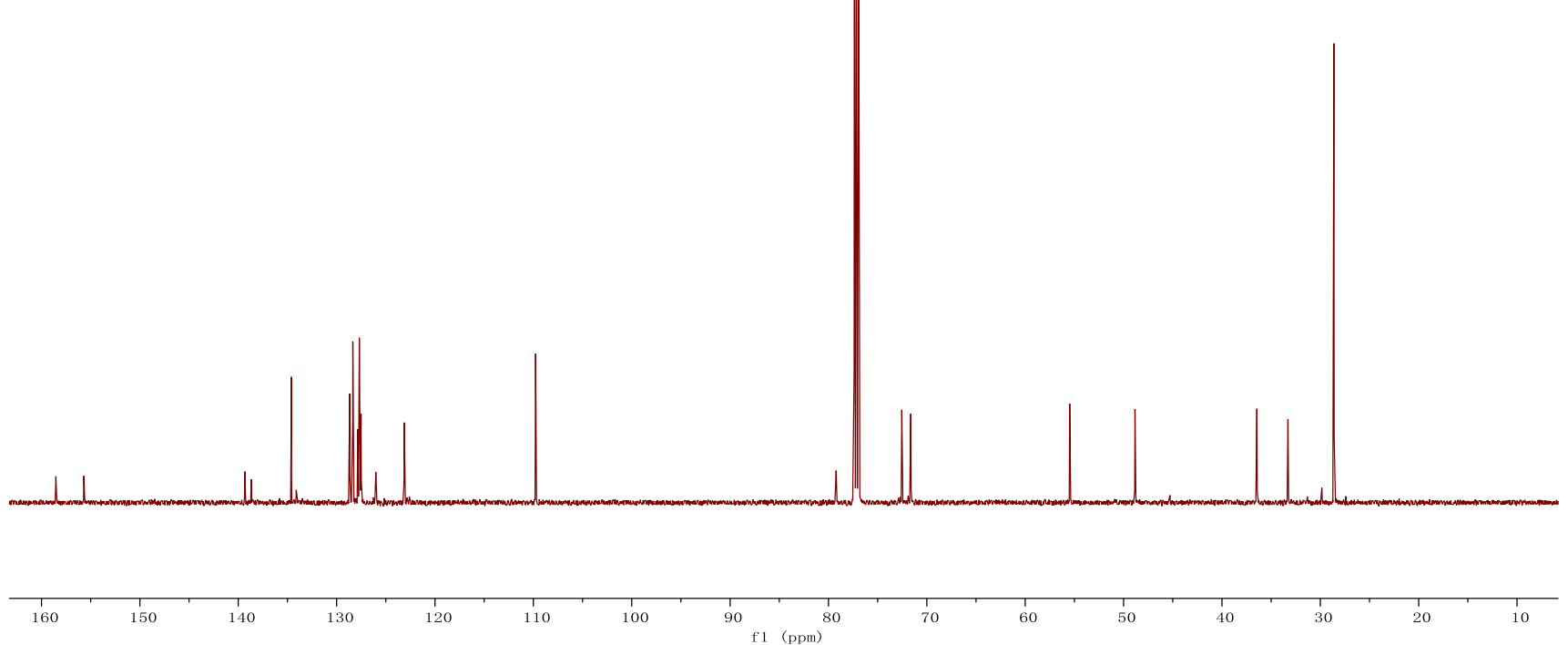


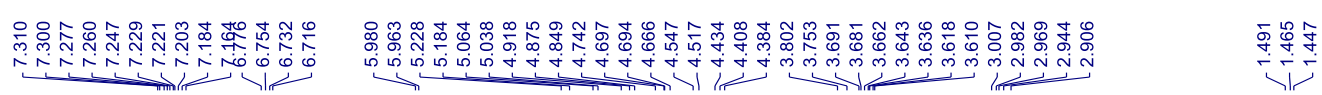

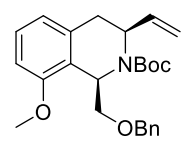

14

${ }^{1} \mathrm{H} \mathrm{NMR} 400 \mathrm{MHz} \mathrm{CDCl}_{3}$

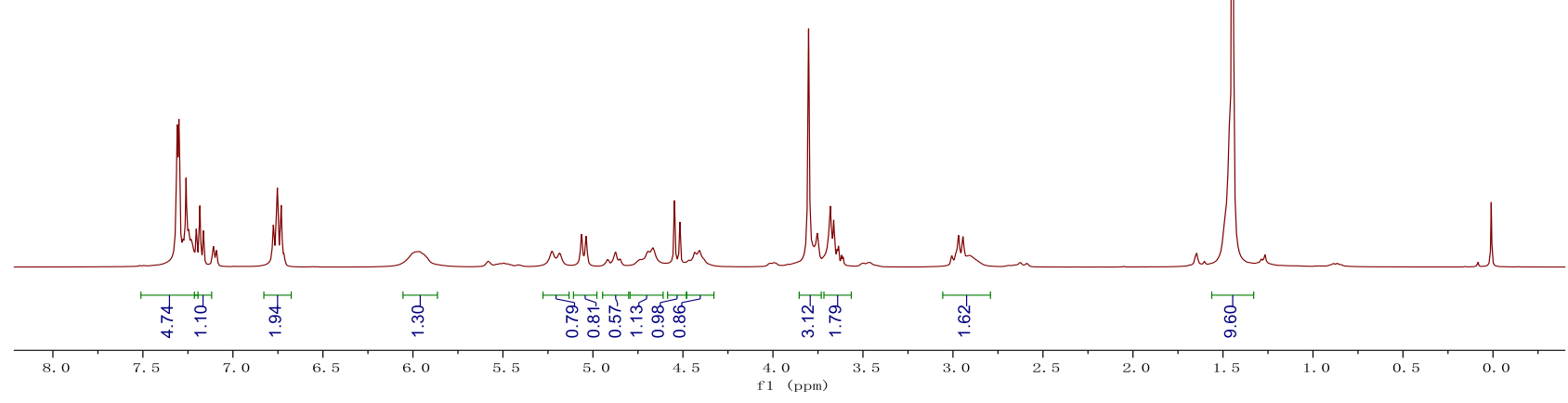

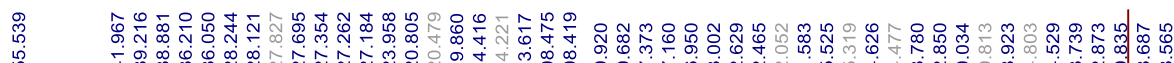

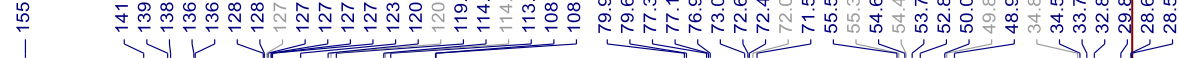

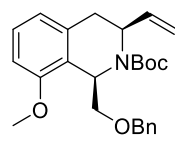

14

${ }^{13} \mathrm{C} \mathrm{NMR} 100 \mathrm{MHz} \mathrm{CDCl}_{3}$

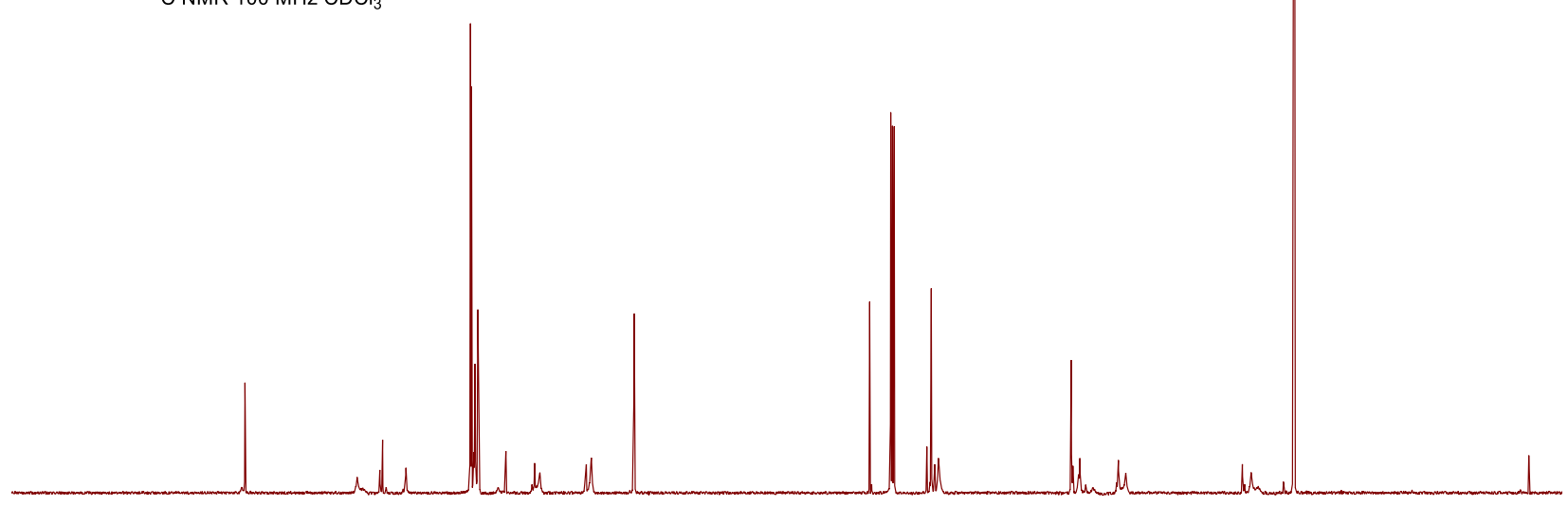

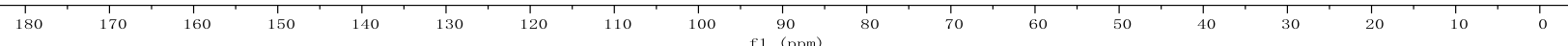




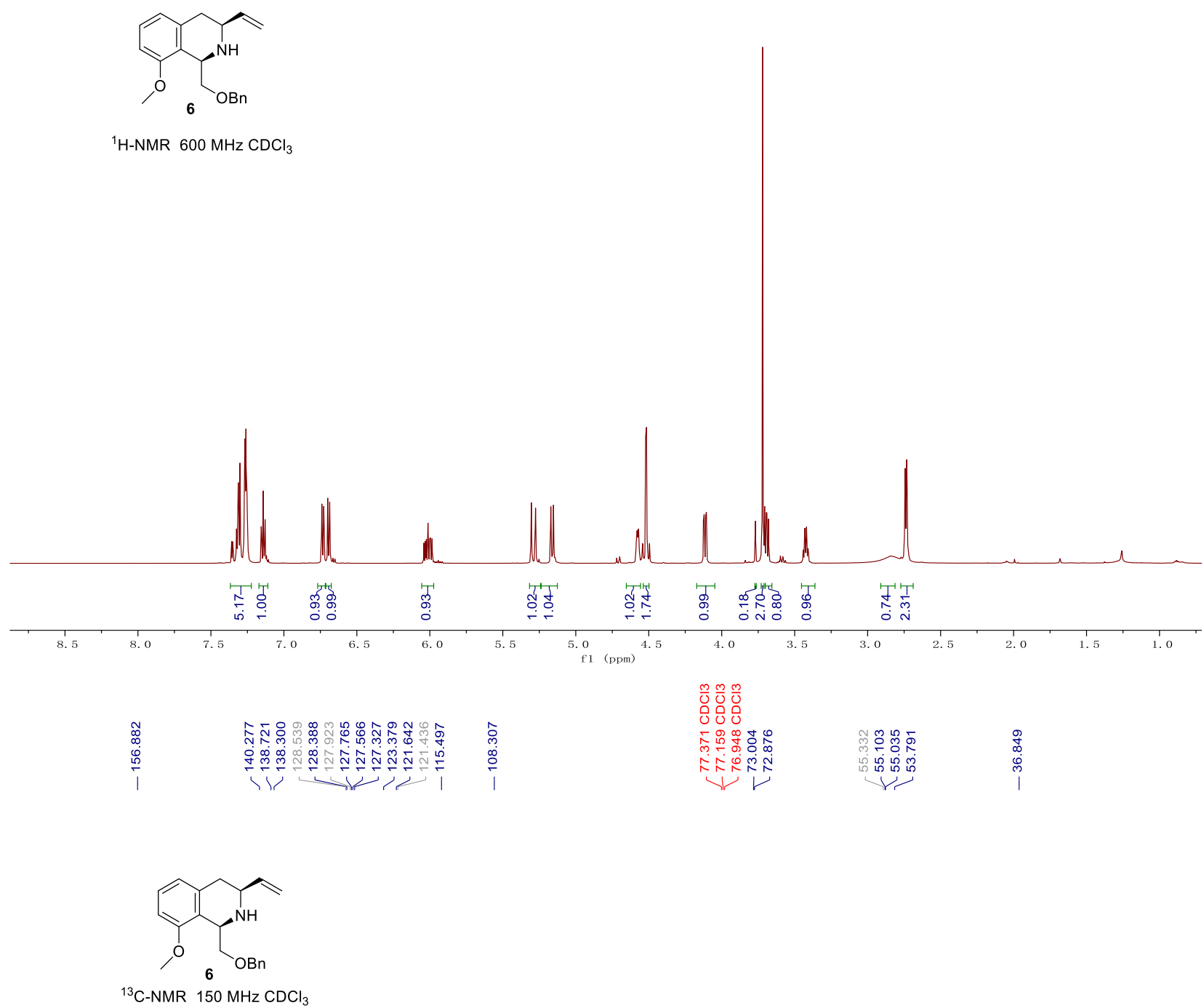

${ }^{1} \mathrm{H}-\mathrm{NMR} 600 \mathrm{MHz} \mathrm{CDCl}_{3}$

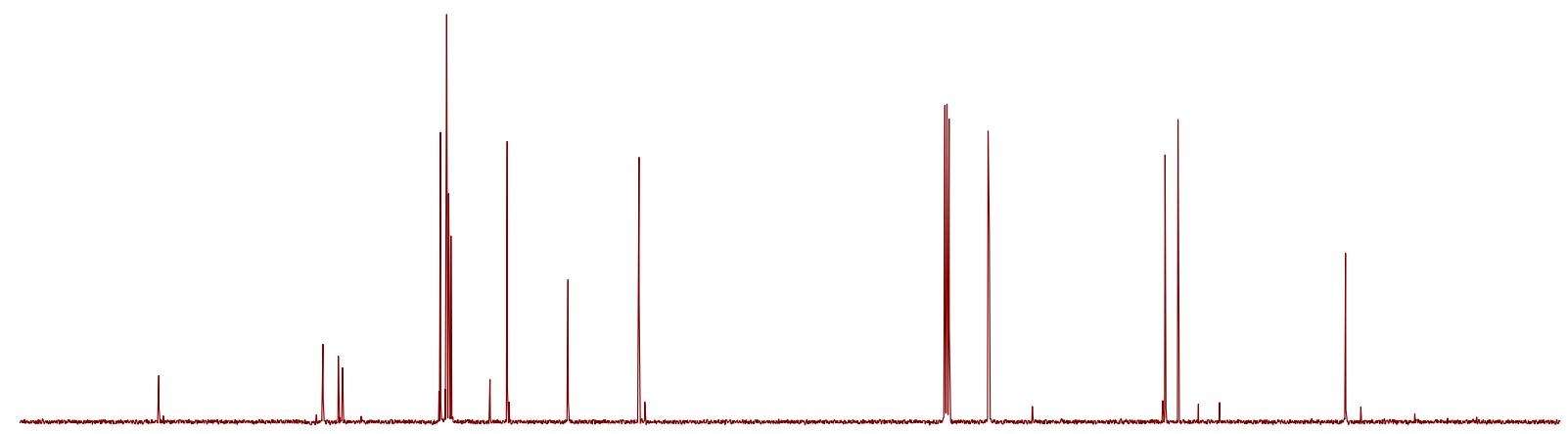

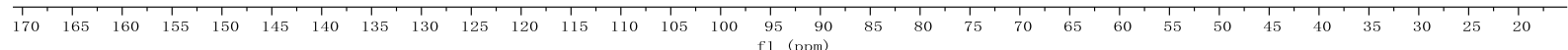



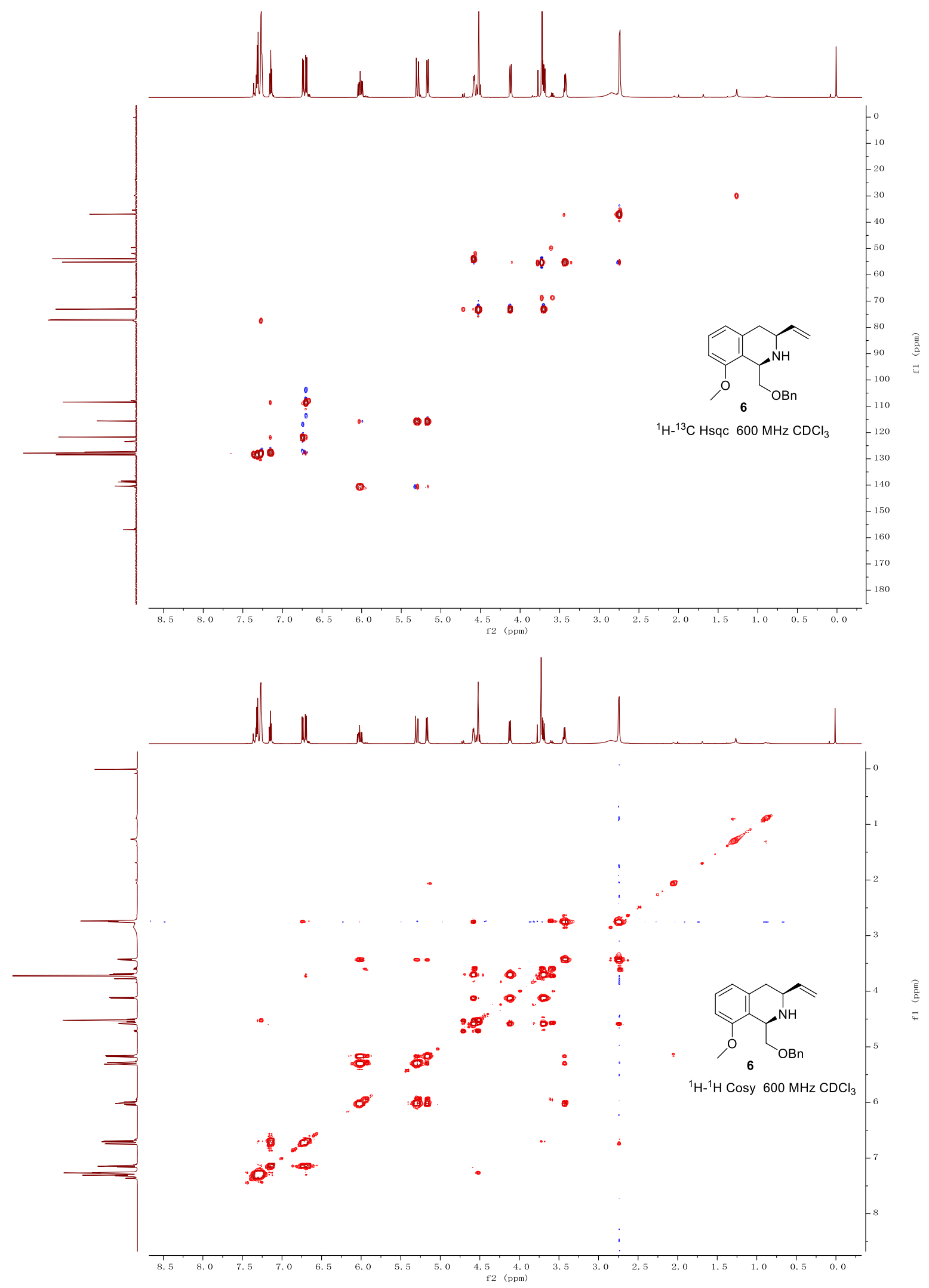

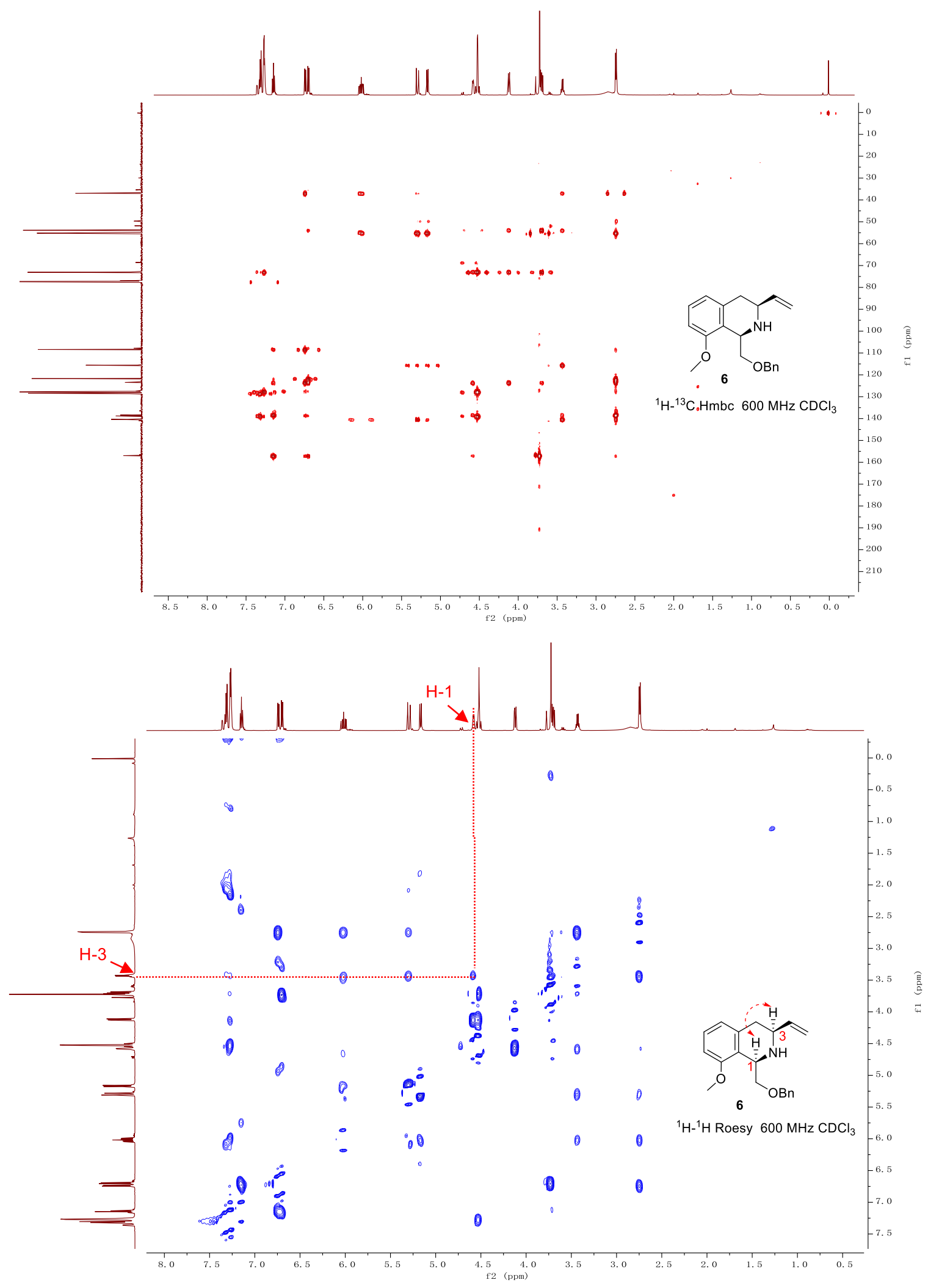


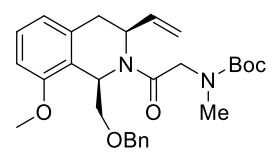

15

${ }^{1} \mathrm{H}$ NMR $400 \mathrm{MHz} \mathrm{CDCl}_{3}$
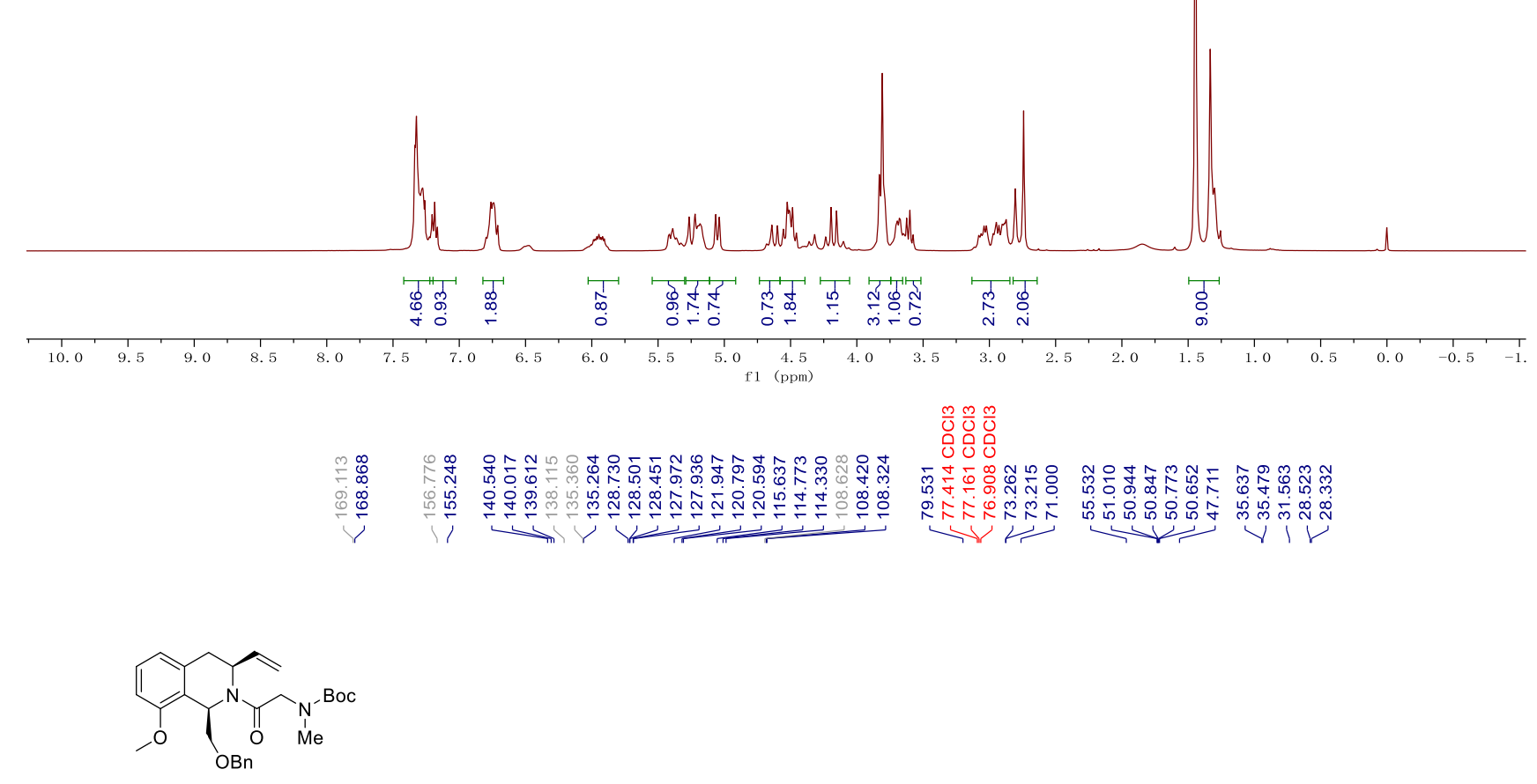

15

${ }^{13} \mathrm{C} \mathrm{NMR} 125 \mathrm{MHz} \mathrm{CDCl}_{3}$
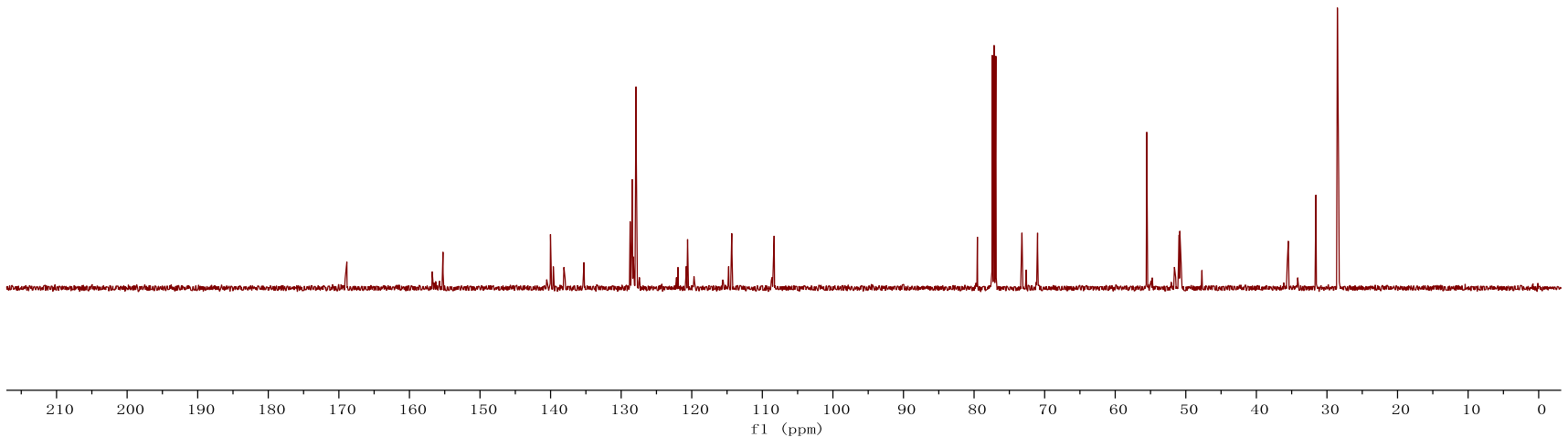


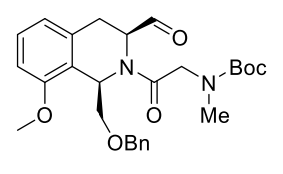

${ }^{1} \mathrm{H} \mathrm{NMR} 400 \mathrm{MHz} \mathrm{CDCl}_{3}$

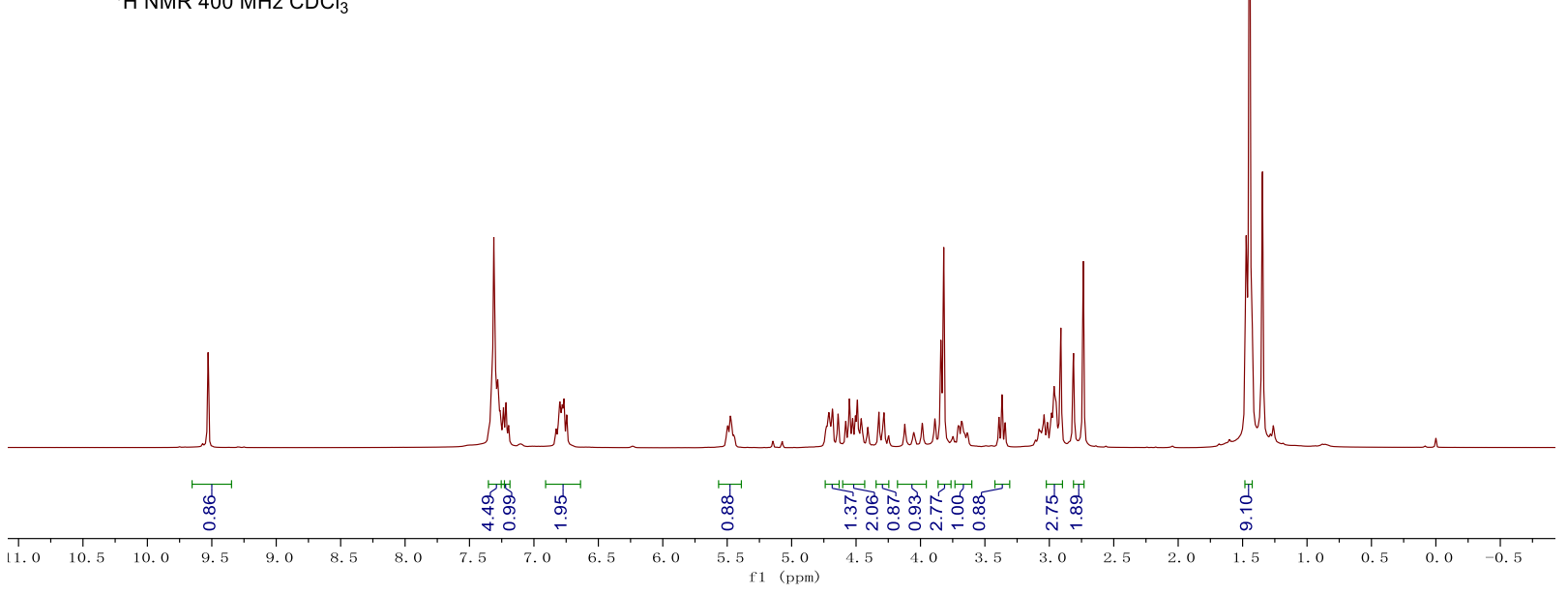

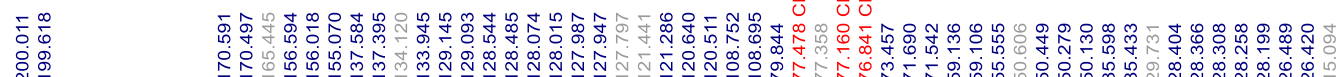

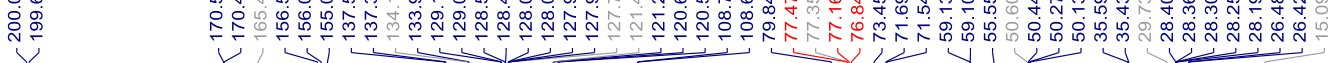

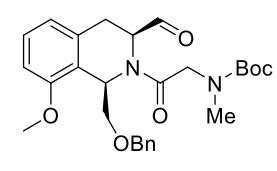

5

${ }^{13} \mathrm{C} \mathrm{NMR} 100 \mathrm{MHz} \mathrm{CDCl}_{3}$

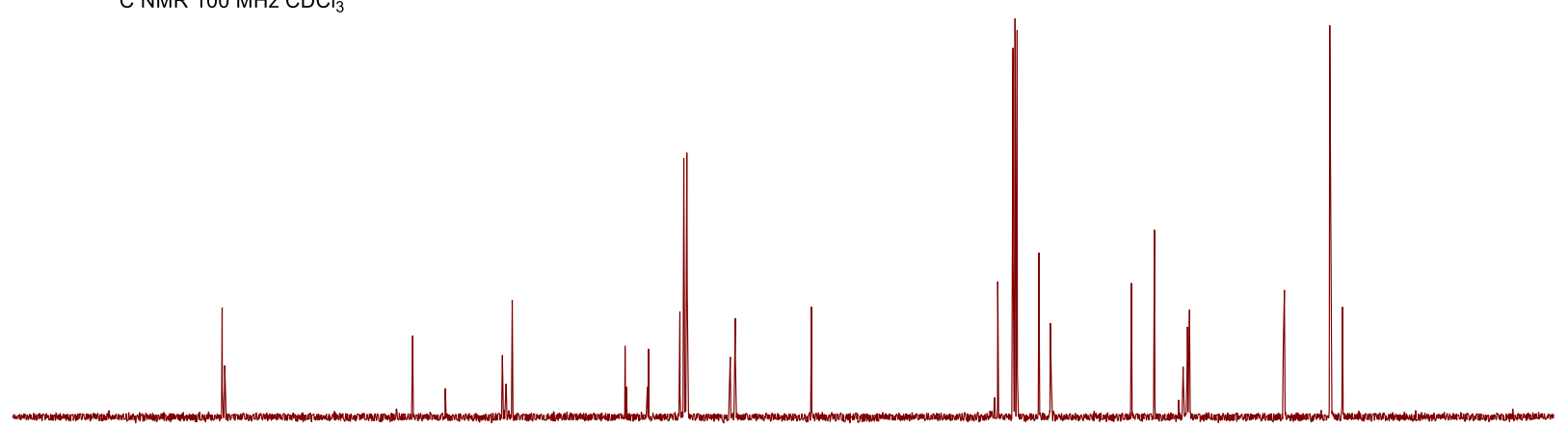

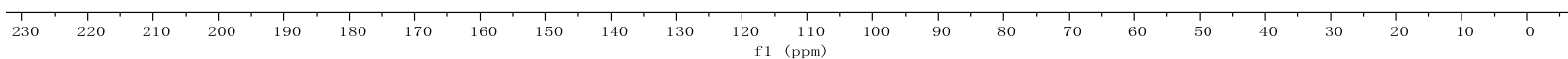




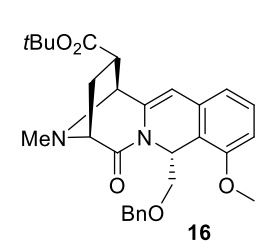

${ }^{1} \mathrm{H} \mathrm{NMR} 400 \mathrm{MHz} \mathrm{CDCl}_{3}$
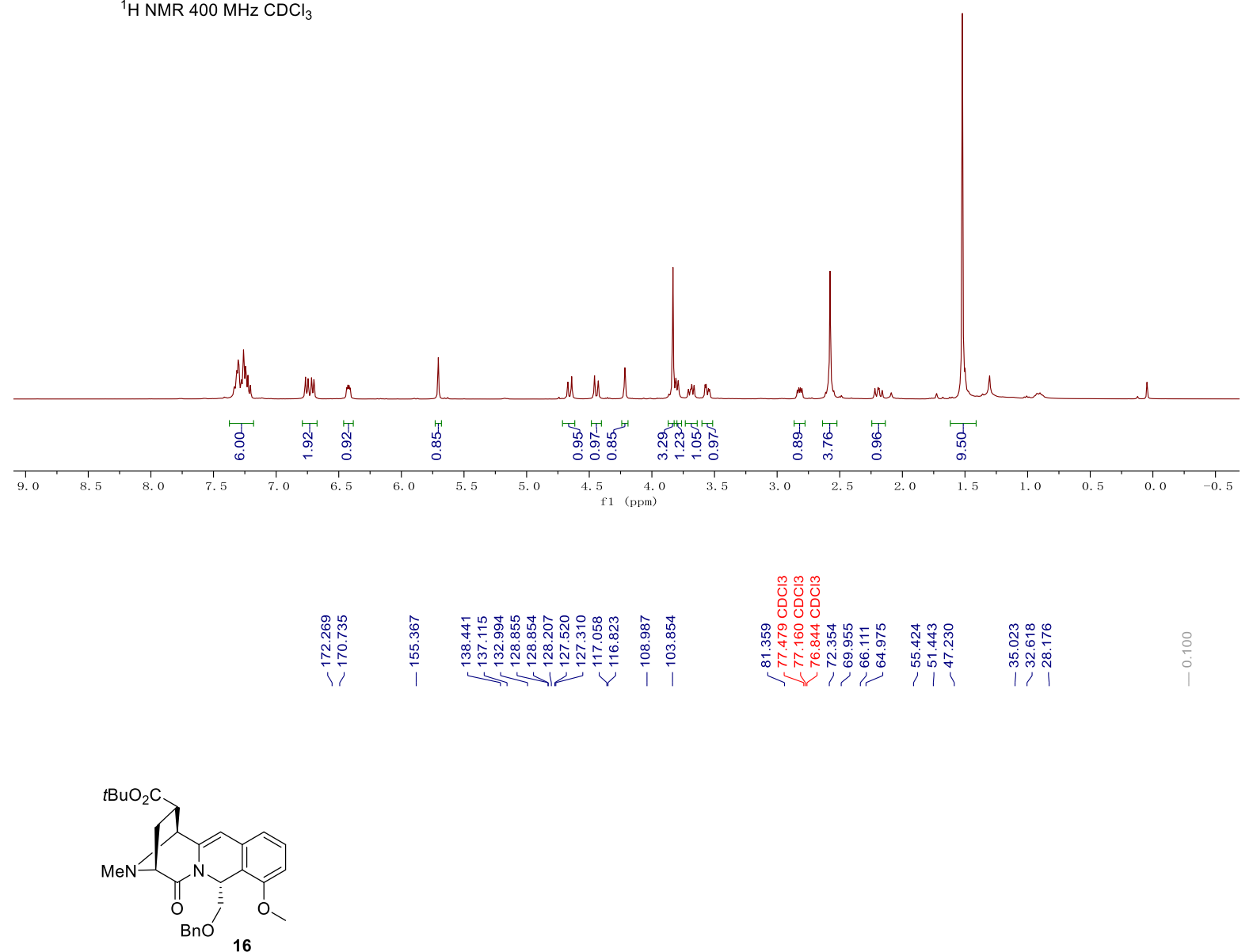

${ }^{13} \mathrm{C} \mathrm{NMR} 100 \mathrm{MHz} \mathrm{CDCl}_{3}$

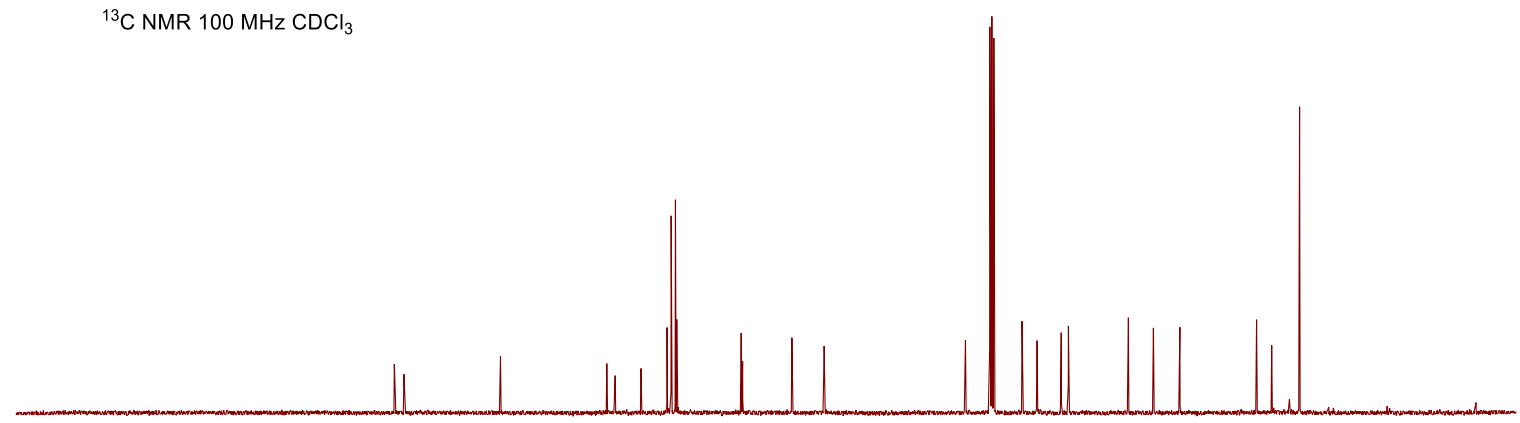

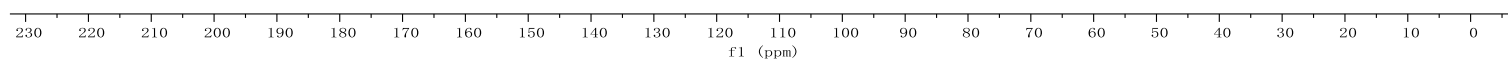


学

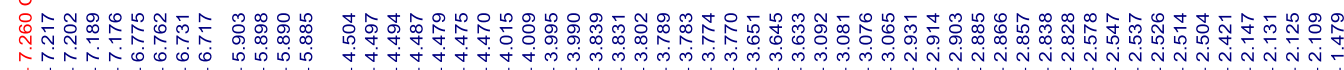

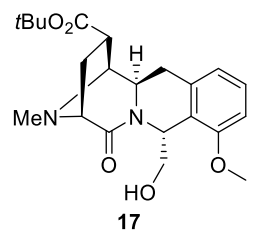

${ }^{1} \mathrm{H}$ NMR $400 \mathrm{MHz} \mathrm{CDCl}_{3}$

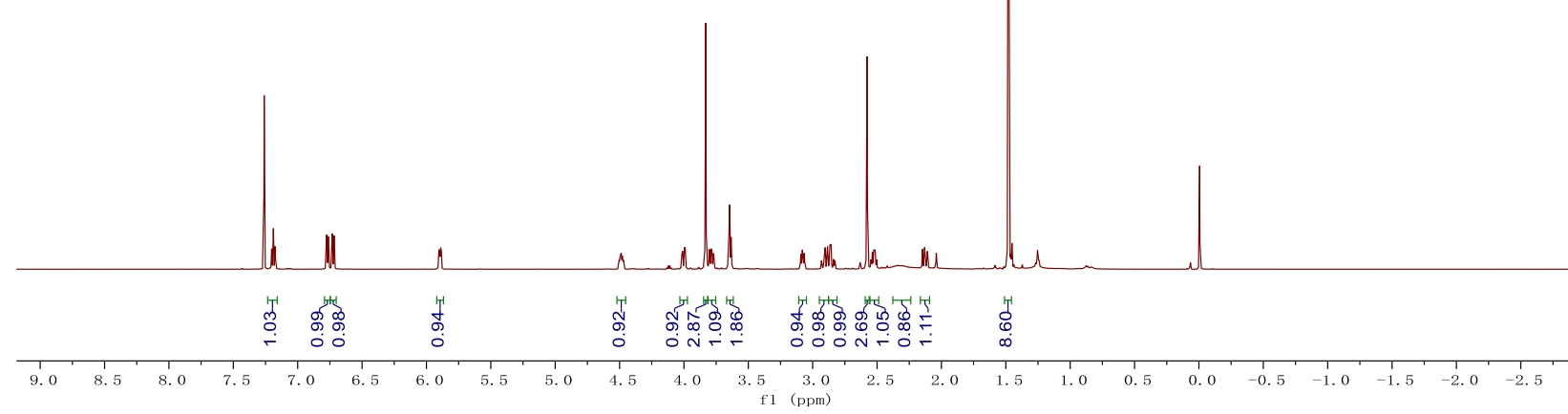

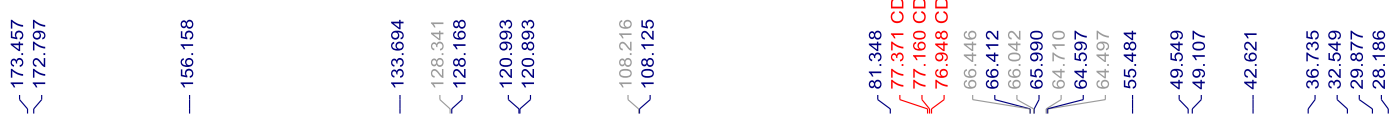

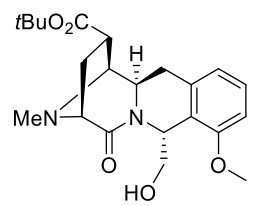

17

${ }^{13} \mathrm{C} \mathrm{NMR} 100 \mathrm{MHz} \mathrm{CDCl}_{3}$
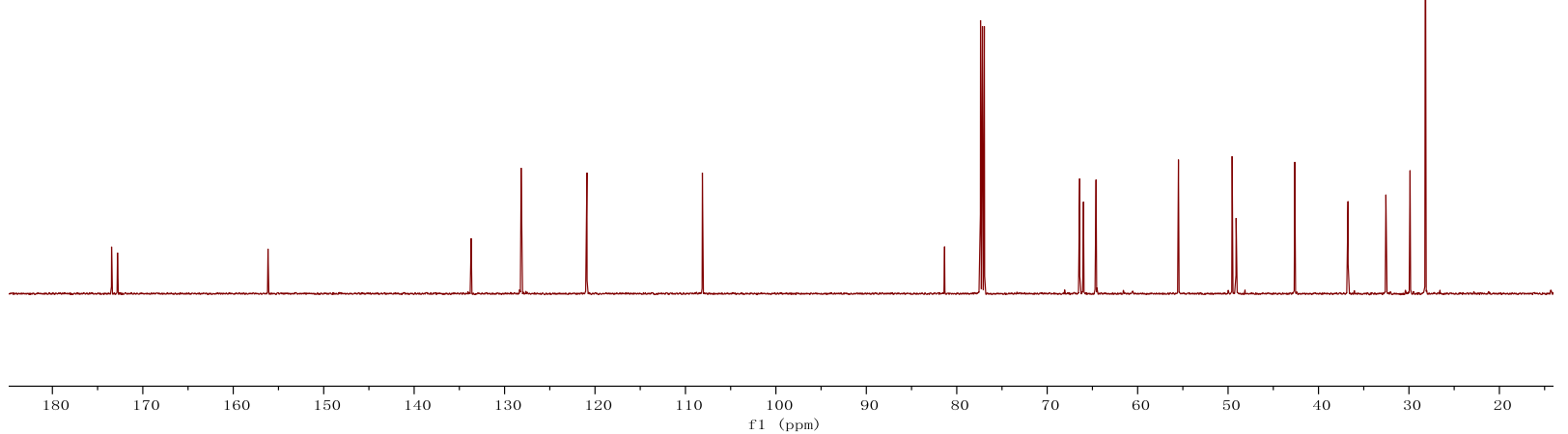


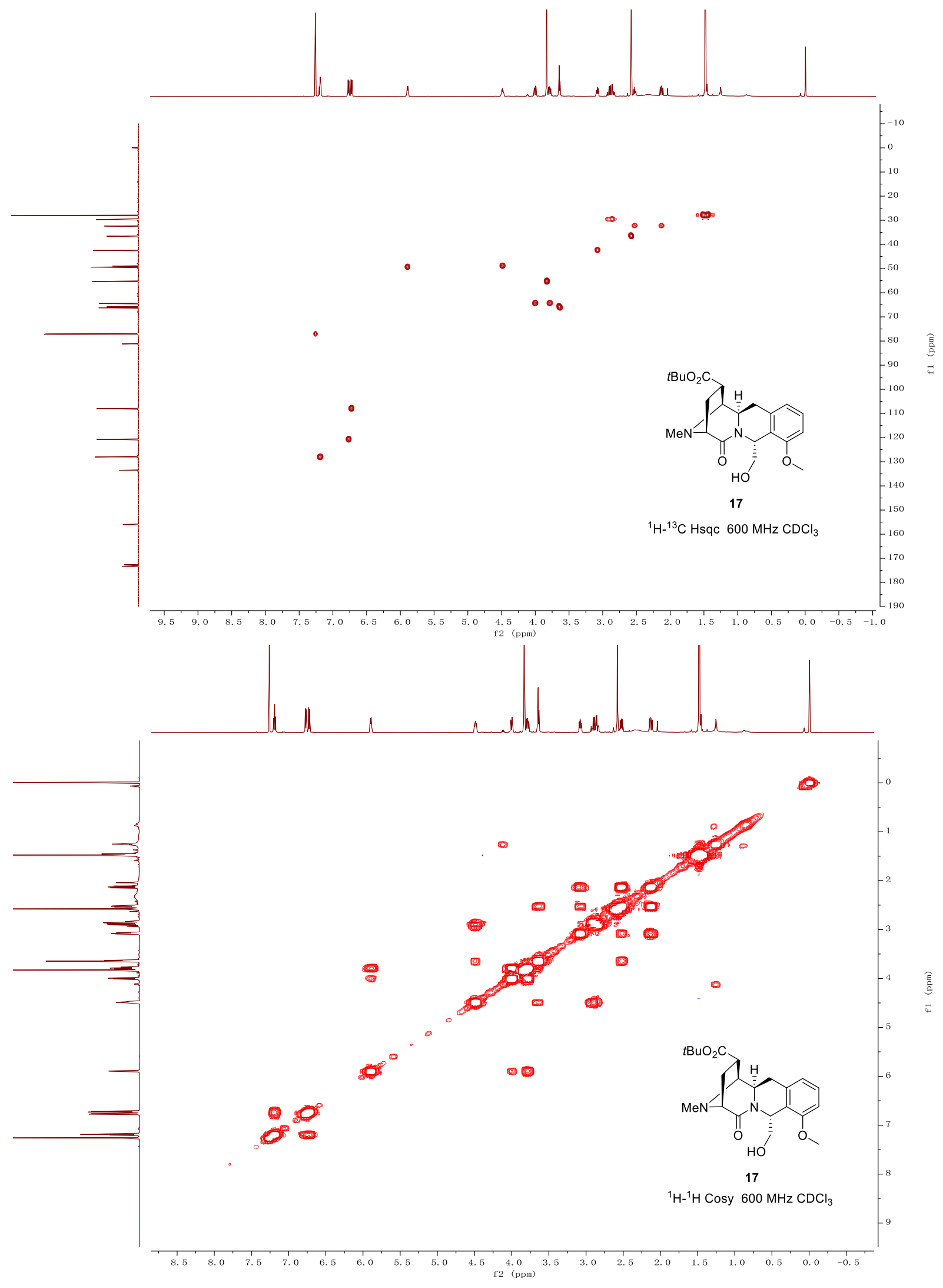




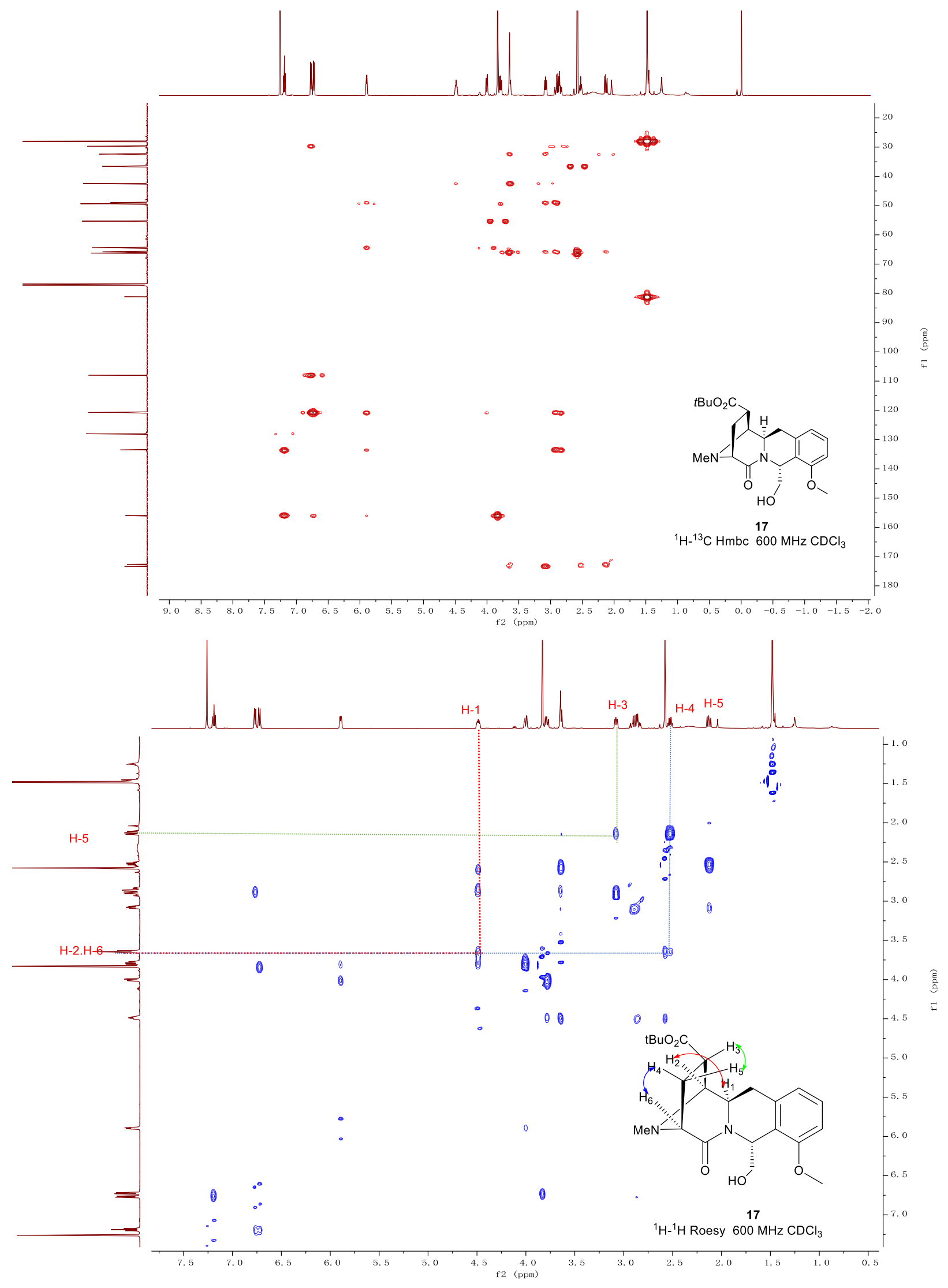




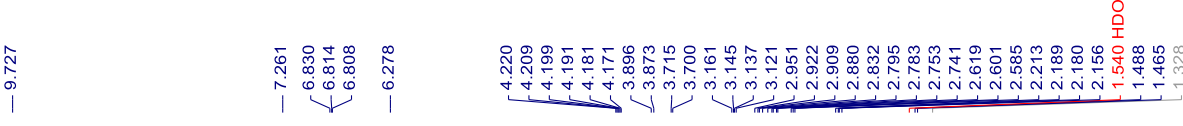

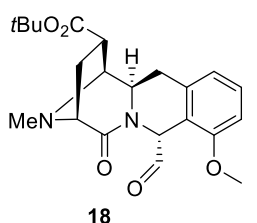

${ }^{1} \mathrm{H}-\mathrm{NMR} 600 \mathrm{MHz} \mathrm{CDCl}_{3}$

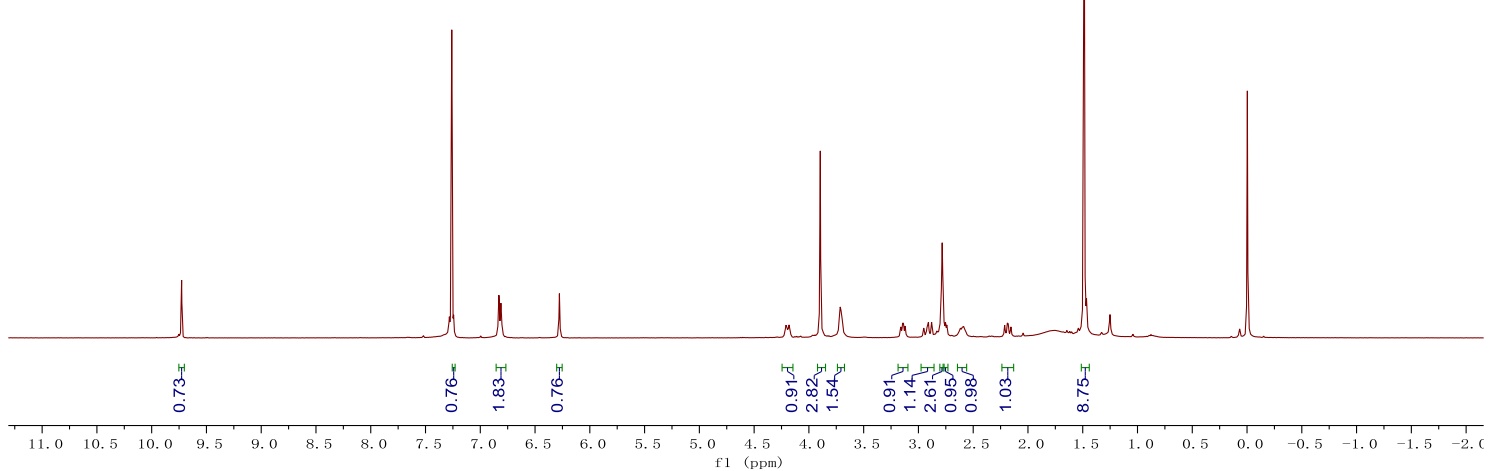

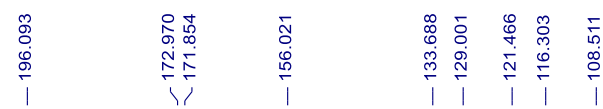

\section{Oै०}

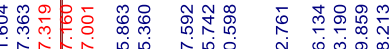

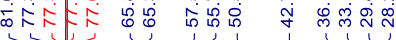

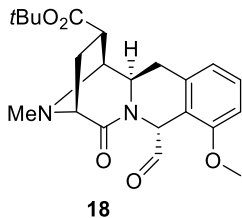

${ }^{13} \mathrm{C}-\mathrm{NMR} 150 \mathrm{MHz} \mathrm{CDCl}_{3}$

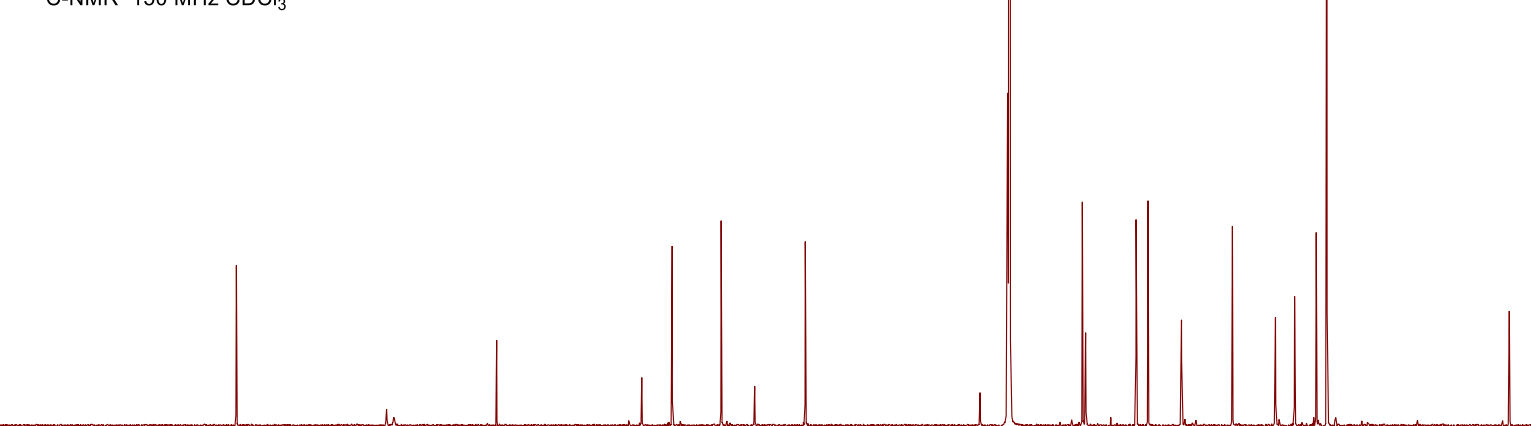

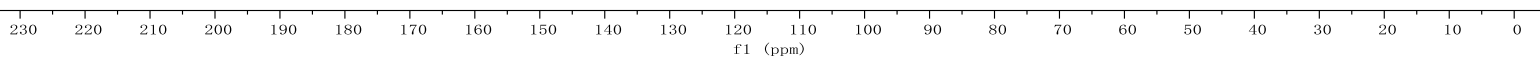




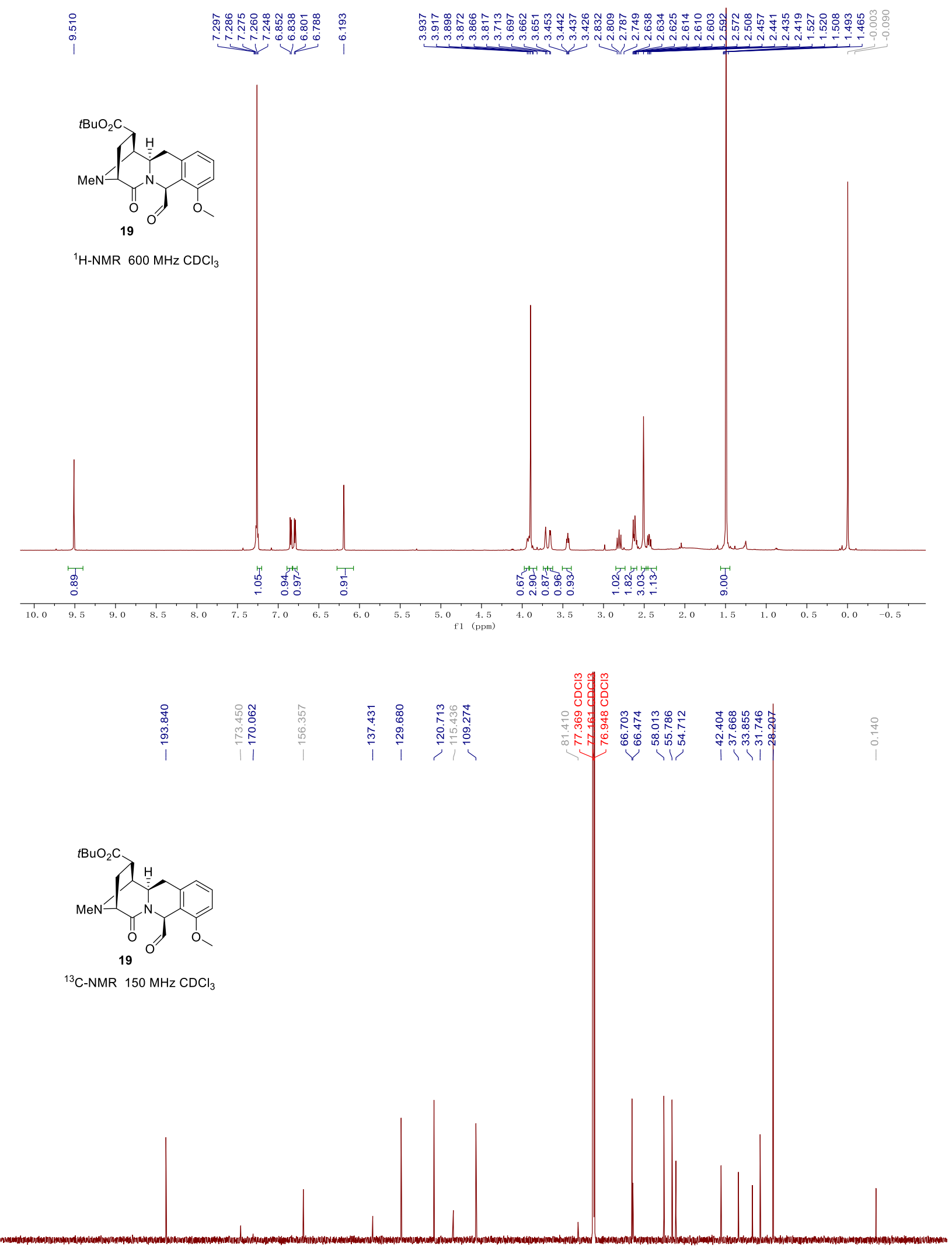

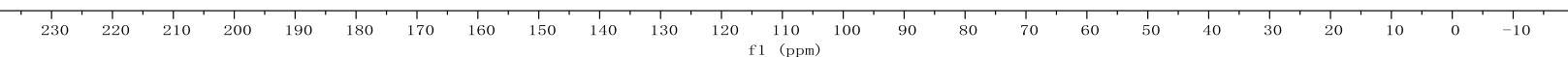



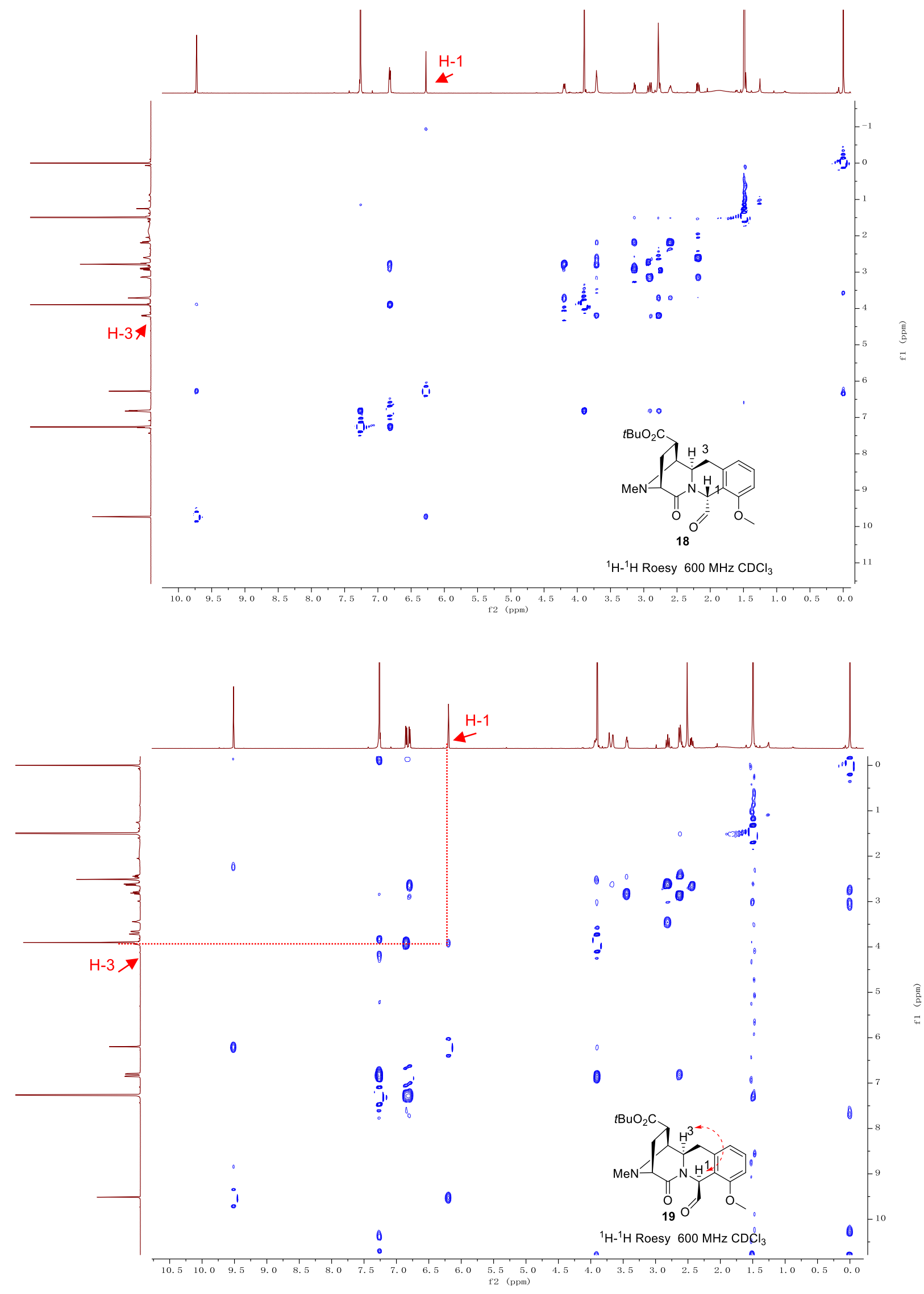
罚

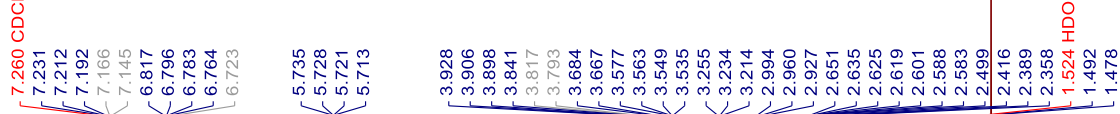

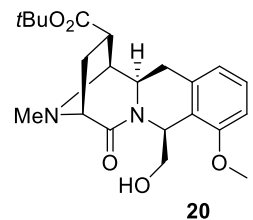

${ }^{1} \mathrm{H} \mathrm{NMR} 400 \mathrm{MHz} \mathrm{CDCl}_{3}$
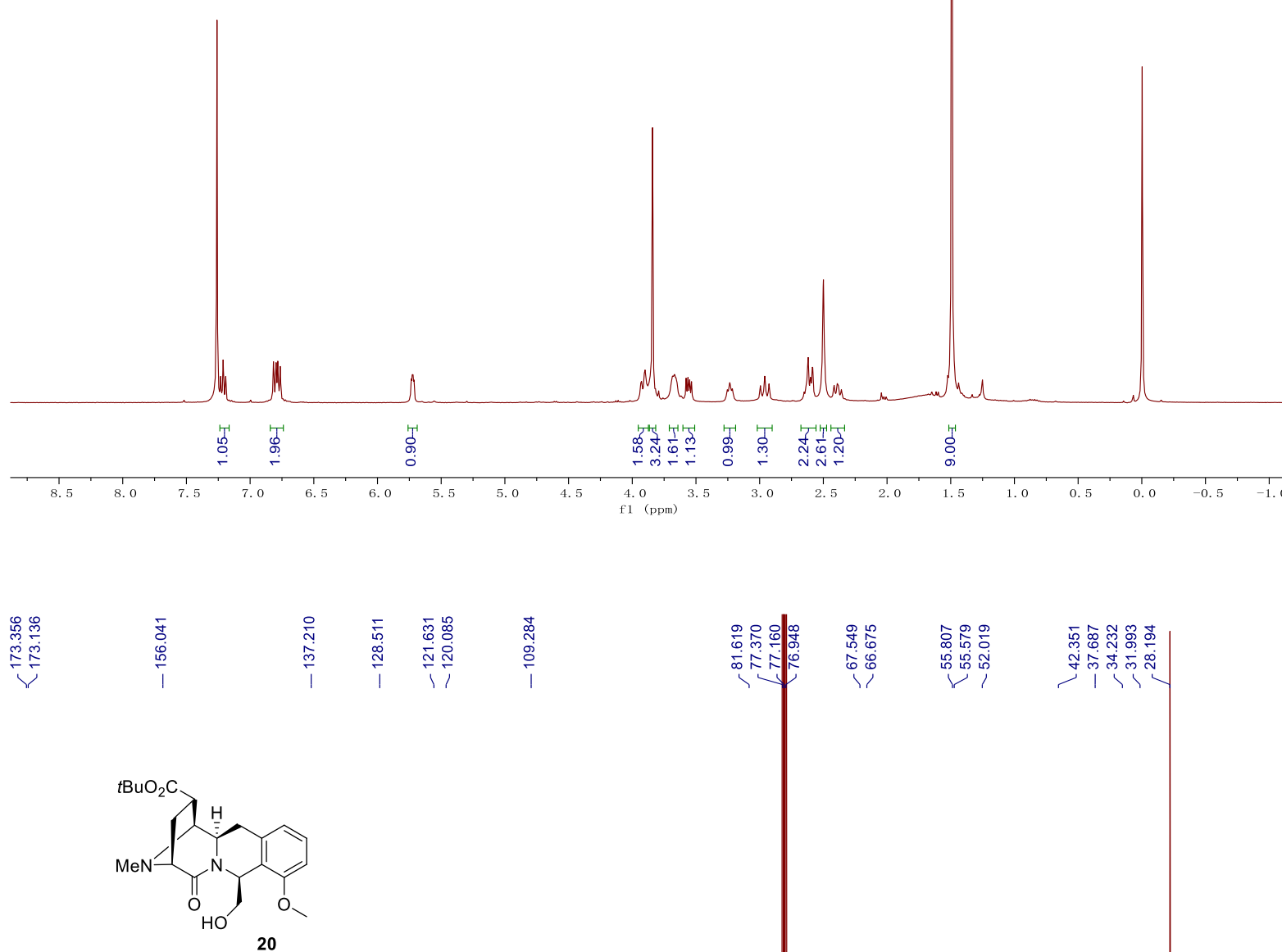

${ }^{13} \mathrm{C} \mathrm{NMR} 150 \mathrm{MHz} \mathrm{CDCl}_{3}$
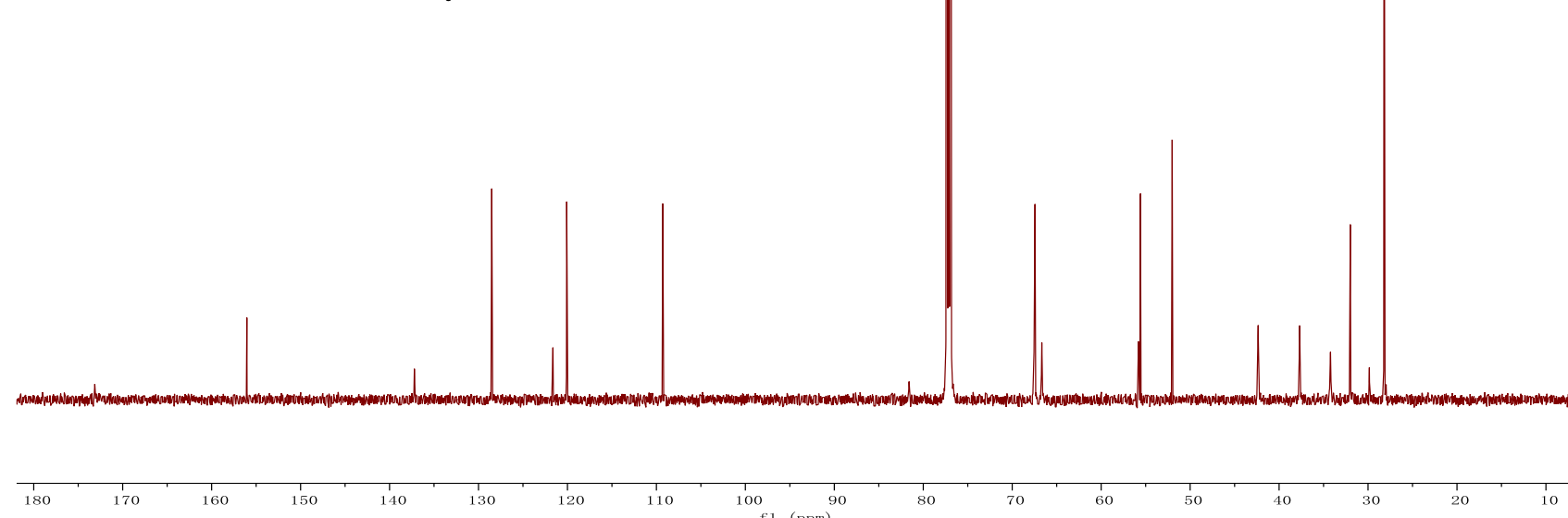


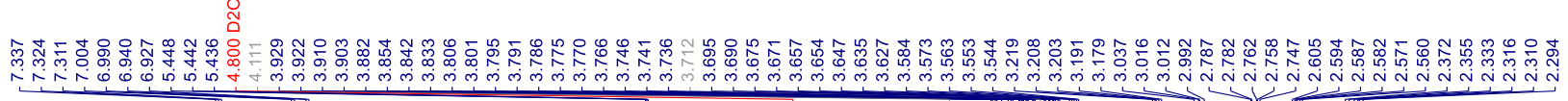

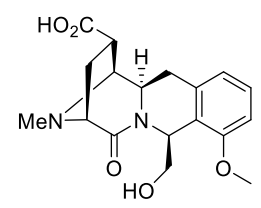

$3(+)$-quinocarcinamide

${ }^{1} \mathrm{H} \mathrm{NMR} 600 \mathrm{MHz} \mathrm{D}_{2} \mathrm{O}$
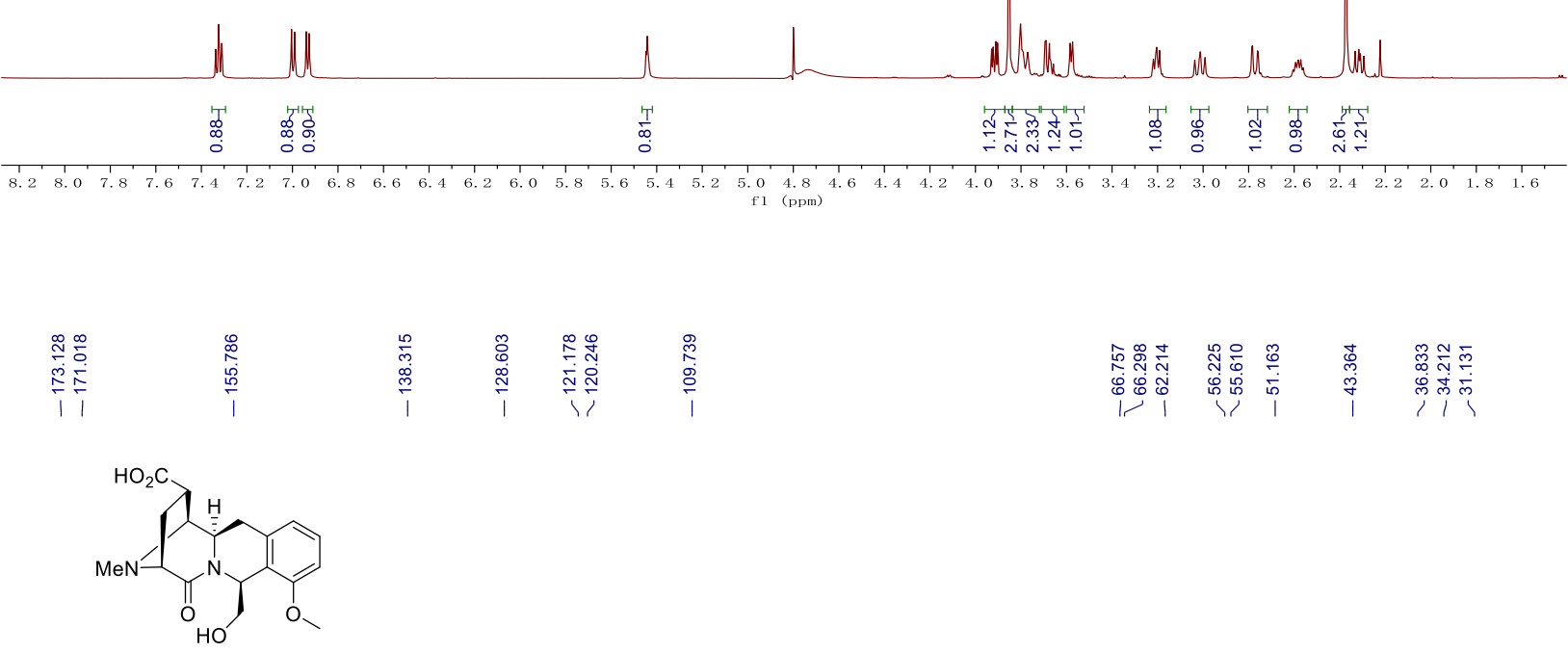

3 (+)-quinocarcinamide

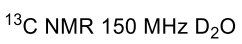
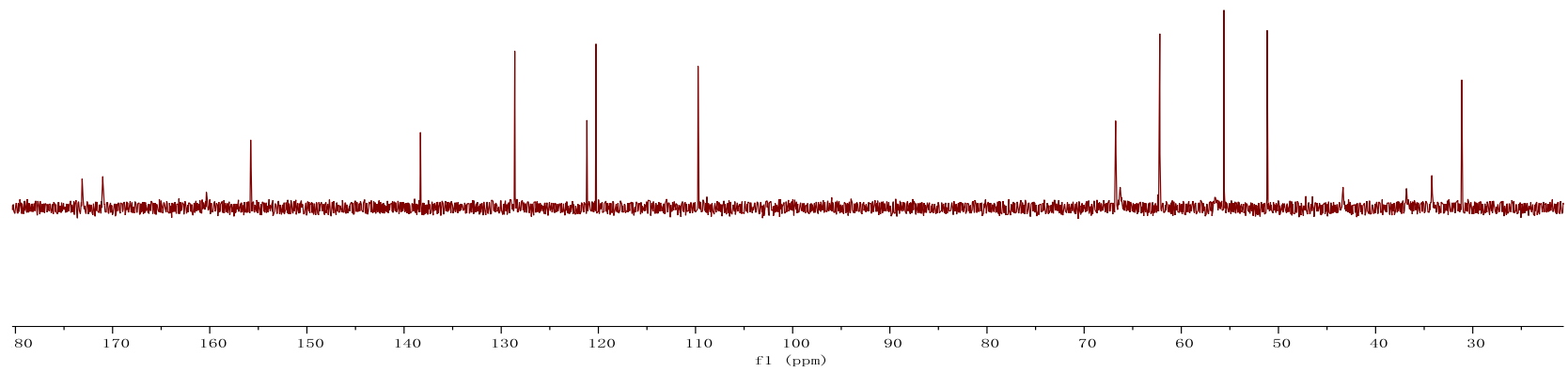

S32 
Comparison of NMR Data $\left(\mathrm{D}_{2} \mathrm{O}\right)$ of Published (-)-quinocarcinamide and Synthetic $(+)-$ quinocarcinamide 3

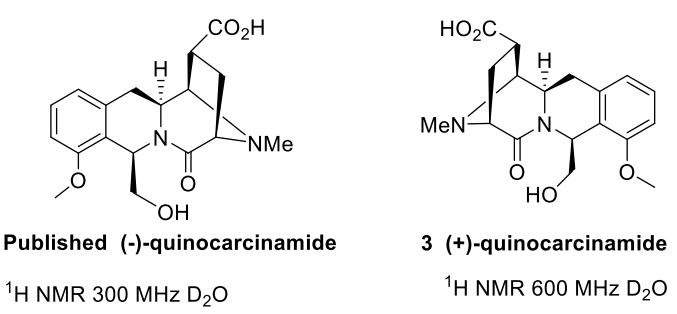

\begin{tabular}{|c|c|c|c|c|}
\hline \multicolumn{2}{|c|}{$\begin{array}{c}\text { Published by Jieping Zhu } \\
\delta_{\mathrm{H}}[\mathrm{ppm}, \mathrm{mult}, J(\mathrm{~Hz})] \\
300 \mathrm{MHz}\end{array}$} & \multicolumn{2}{|c|}{$\begin{array}{c}\text { Synthetic } \\
\delta_{\mathrm{H}}[\mathrm{ppm}, \mathrm{mult}, J(\mathrm{~Hz})] \\
600 \mathrm{MHz}\end{array}$} & \multirow{2}{*}{$\begin{array}{c}\text { Err } \\
\begin{array}{c}\text { (published-Synthetic) } \\
\Delta \delta_{\mathrm{H}}(\mathrm{ppm})\end{array} \\
0.01\end{array}$} \\
\hline 7.33 & $1 \mathrm{H}, \mathrm{t}, 7.8$ & 7.32 & $1 \mathrm{H}, \mathrm{t}, 7.9$ & \\
\hline 7.04 & $1 \mathrm{H}, \mathrm{d}, 7.8$ & 7.02 & $1 \mathrm{H}, \mathrm{d}, 7.9$ & 0.02 \\
\hline 6.94 & $1 \mathrm{H}, \mathrm{d}, 7.8$ & 6.93 & $1 \mathrm{H}, \mathrm{d}, 7.9$ & 0.01 \\
\hline 5.45 & $1 \mathrm{H}, \mathrm{t}, 3.9$ & 5.44 & $1 \mathrm{H}, \mathrm{t}, 3.6$ & 0.01 \\
\hline 3.93 & $\begin{array}{c}1 \mathrm{H}, \mathrm{dd}, 11.2, \\
4.5\end{array}$ & 3.92 & $1 \mathrm{H}, \mathrm{dd}, 11.5,4.3$ & 0.01 \\
\hline 3.87 & $3 \mathrm{H}, \mathrm{s}$ & 3.85 & $3 \mathrm{H}, \mathrm{s}$ & 0.02 \\
\hline $\begin{array}{c}3.83- \\
3.74\end{array}$ & $2 \mathrm{H}, \mathrm{m}$ & $\begin{array}{r}3.84- \\
3.72\end{array}$ & $2 \mathrm{H}, \mathrm{m}$ & 0.01 \\
\hline 3.70 & $\begin{array}{c}1 \mathrm{H}, \mathrm{dd}, 11.2, \\
4.5\end{array}$ & 3.71 & $1 \mathrm{H}, \mathrm{dd}, 11.5,3.0$ & 0.01 \\
\hline 3.58 & $1 \mathrm{H}, \mathrm{d}, 6.6$ & 3.58 & $1 \mathrm{H}, \mathrm{d}, 6.4$ & 0 \\
\hline 3.22 & $1 \mathrm{H}, \mathrm{dd}, 10.2,6.9$ & 3.21 & $1 \mathrm{H}, \mathrm{dd}, 9.7,6.6$ & 0.01 \\
\hline $\begin{array}{c}3.06- \\
3.00 \\
\end{array}$ & $1 \mathrm{H}, \mathrm{m}$ & 3.01 & $1 \mathrm{H}, \mathrm{dd}, 14.8,12.3$ & 0 \\
\hline $\begin{array}{c}2.82- \\
2.74 \\
\end{array}$ & $1 \mathrm{H}, \mathrm{m}$ & 2.77 & $1 \mathrm{H}, \mathrm{dd}, 14.8,2.4$ & 0 \\
\hline 2.59 & $\begin{array}{c}\mathrm{H}, \\
\mathrm{ddd}, 13.6,6.9 \\
6.9 \\
\end{array}$ & 2.58 & $\begin{array}{c}1 \mathrm{H}, \mathrm{ddd}, 13.3,6.6 \text {, } \\
6.6,\end{array}$ & 0.01 \\
\hline 2.38 & $3 \mathrm{H}, \mathrm{s}$ & 2.37 & $3 \mathrm{H}, \mathrm{s}$ & 0.01 \\
\hline 2.34 & $1 \mathrm{H}, \mathrm{dd} 13.6,9.9$ & 2.32 & $1 \mathrm{H}, \mathrm{dd}, 13.3,9.8$ & 0.02 \\
\hline
\end{tabular}


Part of the original HRMS analysis reports.

Qualitative Analysis Report

\begin{tabular}{|c|c|c|c|}
\hline Data Filename & AS.01143d & Sample Name & ASLO1143 \\
\hline Sample Type & Sample & Position & P1-A4 \\
\hline Instrument Name & Instrument 1 & User Name & \\
\hline Acq Method & $\mathrm{sm}$ & Acquired Time & $11 / 5 / 20201: 55: 49 \mathrm{PM}$ \\
\hline $\begin{array}{l}\text { IRM Calibration Status } \\
\text { Comment }\end{array}$ & s.coess & DA Method & Defaut:m \\
\hline Sample Group & & & \\
\hline $\begin{array}{l}\text { Acquisition SW } \\
\text { Version }\end{array}$ & $\begin{array}{l}\text { les TOF/65C0 series } \\
.05 .01 \text { (BS125.2) }\end{array}$ & & \\
\hline
\end{tabular}<smiles>C=C[C@H](NC(=O)OC(C)(C)C)c1c(Br)cccc1OC</smiles>

User Spectra

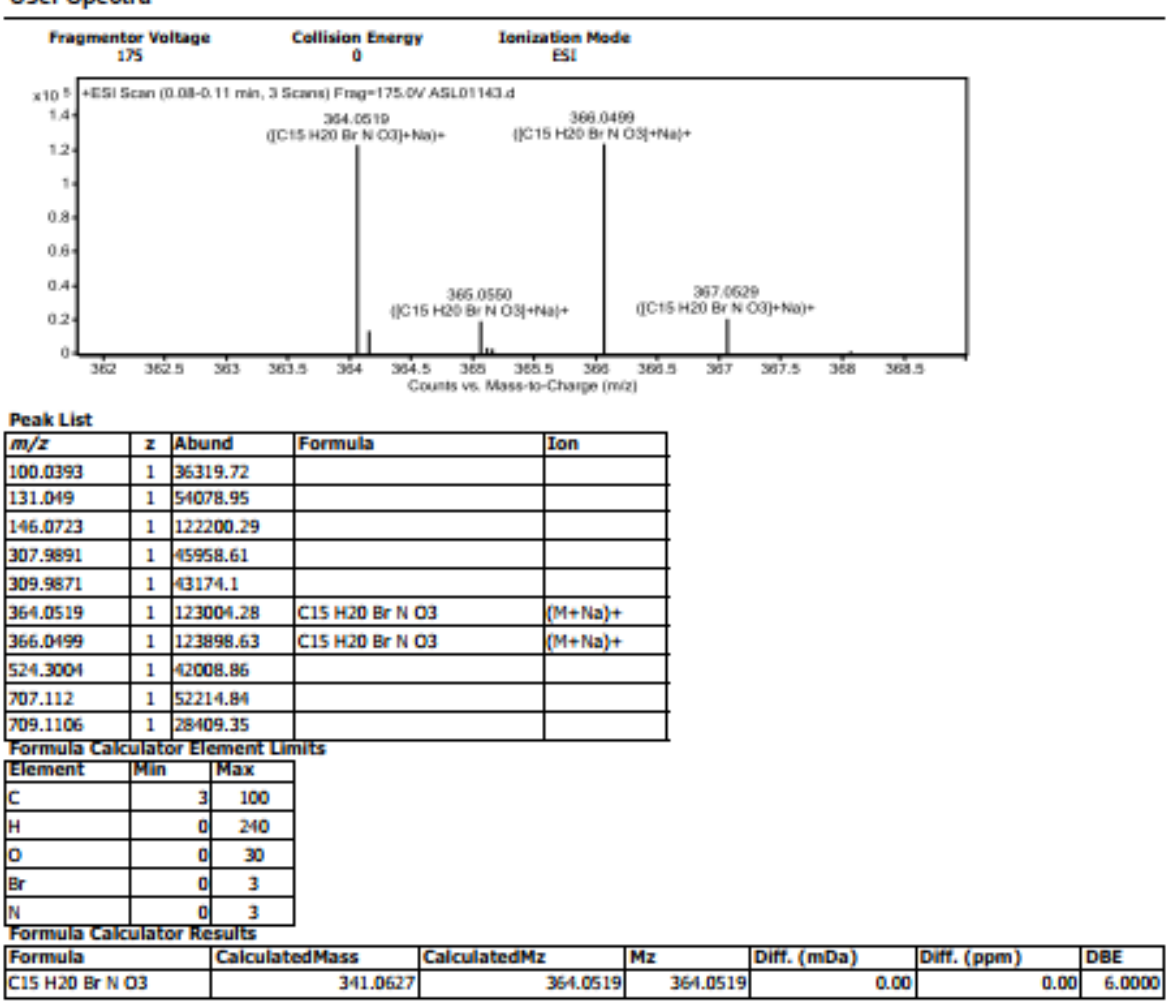

-- End of Report - 
Qualitative Analysis Report

\begin{tabular}{|c|c|c|c|}
\hline Data Filename & al070076.d & Sample Name & aldo7076 \\
\hline Sample Type & Sample & Position & P1-A1 \\
\hline Instrument Name & Instrument: 1 & User Name & \\
\hline Acq Method & $\mathrm{sm}$ & Acquired Time & $11 / 24 / 2020$ 1:54:07 PM \\
\hline $\begin{array}{l}\text { IRM Calibration Status } \\
\text { Comment }\end{array}$ & s.coess & DA Method & Default.m \\
\hline Sample Group & & & \\
\hline $\begin{array}{l}\text { Acquisition SW } \\
\text { Version }\end{array}$ & $\begin{array}{l}\text { les TOEF/6500 series } \\
05.01(\mathrm{BS} 125.2)\end{array}$ & & \\
\hline
\end{tabular}<smiles>C=CC1Cc2cccc(OC)c2C(COCc2ccccc2)N1</smiles>

6

User Spectra
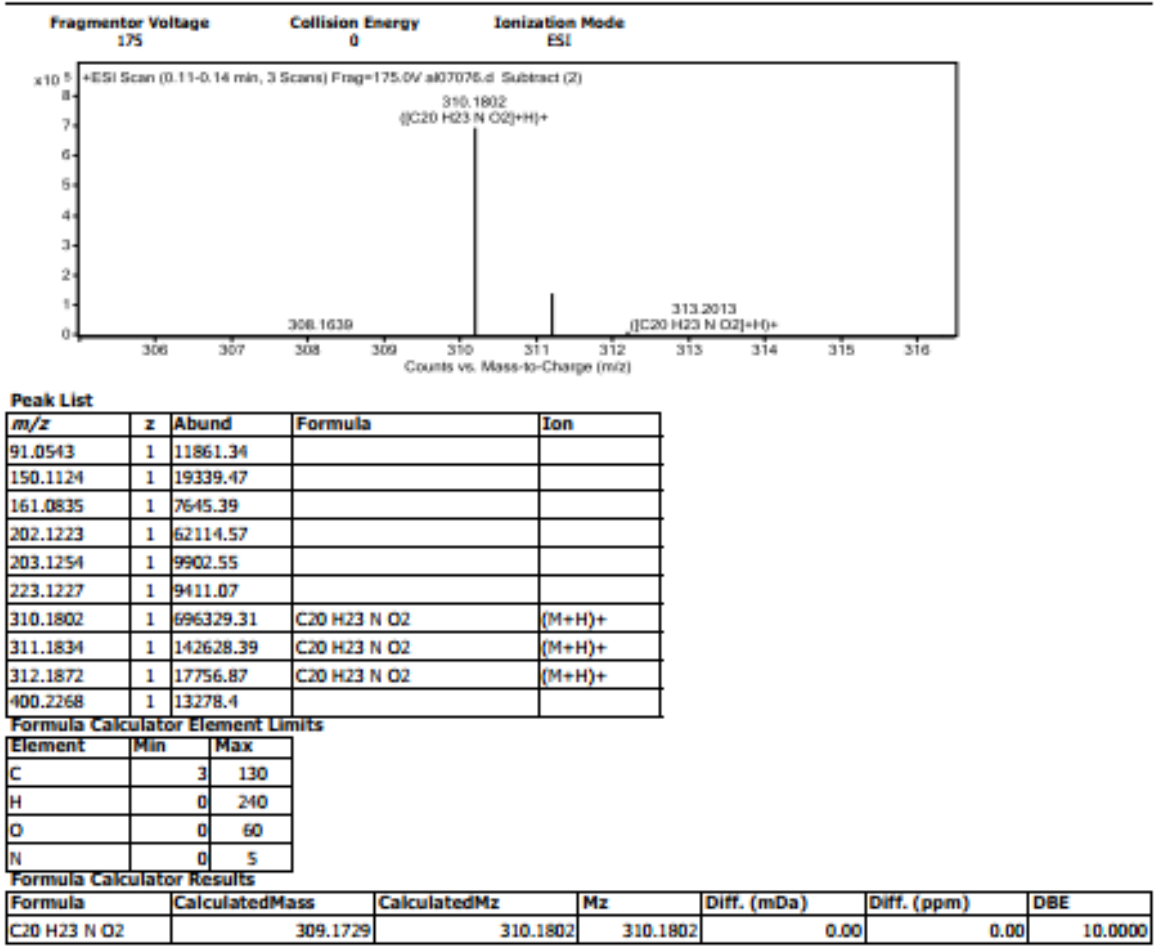

$\mathrm{C} 20 \mathrm{H}_{23} \mathrm{~N} \mathrm{O}_{2}$ 309.1729 310.1802

-- End of Report -. 
Qualitative Analysis Report

\begin{tabular}{|c|c|c|c|}
\hline \multirow{2}{*}{$\begin{array}{l}\text { Data Filename } \\
\text { Sample Type }\end{array}$} & ad07113.d & Sample Name & al07113 \\
\hline & Sample & Position & P1-A1 \\
\hline Instrument Name & Instrument: 1 & User Name & \\
\hline Acq Method & s.m & Acquired Time & $12 / 21 / 20209: 47: 06 \mathrm{AM}$ \\
\hline IRM Calibration Status & Success & DA Method & Defaut.m \\
\hline \multicolumn{4}{|l|}{ Comment } \\
\hline Sample Group & & & \\
\hline $\begin{array}{l}\text { Acquisition SW } \\
\text { Version }\end{array}$ & $\begin{array}{l}\text { es TOF/6500 series } \\
05.01 \text { (BS125.2) }\end{array}$ & & \\
\hline
\end{tabular}

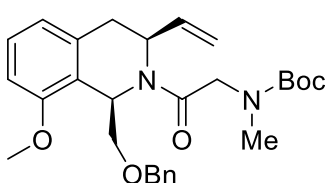

15

User Spectra

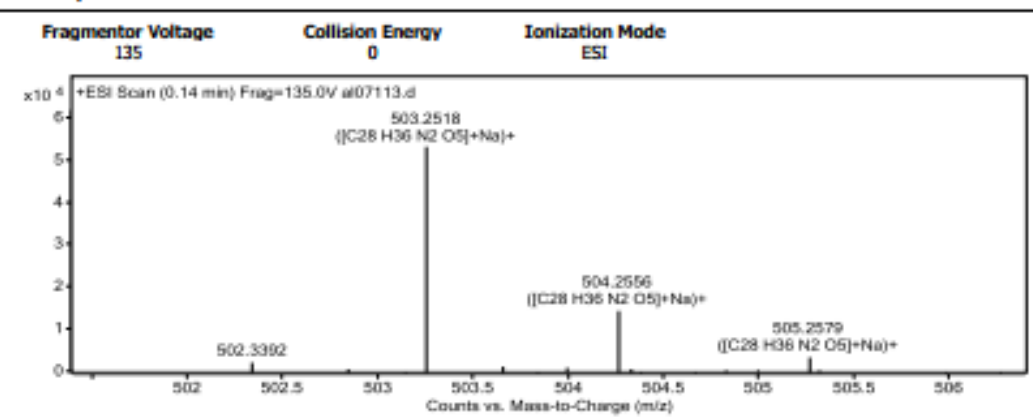

Peak List
\begin{tabular}{|l|r|l|l|l|}
\hline$m / z$ & $z$ & Abund & Formula & Ion \\
\hline 141.1134 & & 16143.36 & & \\
\hline 172.0943 & & 17675.54 & & \\
\hline 288.2901 & 1 & 62023.82 & & \\
\hline 289.2936 & 1 & 12788.62 & & \\
\hline 316.3212 & 1 & 25994.95 & & \\
\hline 481.2703 & 1 & 46130.89 & & \\
\hline 482.2742 & 1 & 14897.38 & & \\
\hline 503.2518 & 1 & 53278.03 & C28 H36 N2 O5 & $(\mathrm{M}+\mathrm{Na})+$ \\
\hline 504.2556 & 1 & 14702.18 & $\mathrm{C} 8 \mathrm{BH} 36 \mathrm{N2}$ O5 & $(\mathrm{M}+\mathrm{Na})+$ \\
\hline 922.0098 & 1 & 19214.38 & & \\
\hline
\end{tabular}

Element

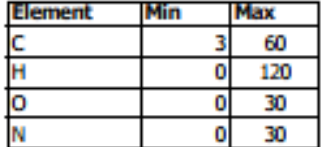

\begin{tabular}{|r|r|r|}
\hline$N$ & 0 & 30 \\
\hline
\end{tabular}

\begin{tabular}{|l|r|r|r|r|r|r|}
\hline Formula & CalculatedMass & CalculatedMz & Mz & Diff. (mba) & Diff. (ppm) & DEEE \\
\hline C28 FB6 N2 05 & 480.2624 & 503.2516 & 503.2518 & -0.20 & -0.40 & 12.0000 \\
\hline
\end{tabular}

-- End or Repart - 
Qualitative Analysis Report

Data Filename

Sample Type

Instrument Name

Acq Method

IRM Calibration Status

Comment

Sample Group

Acquisition SW

Version

\section{a $1060631 . d$}

Sample

Irstrument 1

s.m

Sucres:
Sample Name al060531

Position P1-B1

User Name

Acquired Time $\quad$ 8/24/2020 4:06:39 PM

DA Method Defast.m

Info.

6200 series TOF $/ 6500$ series

Q-TOF B.05.01 (BS125.2)

User Spectra

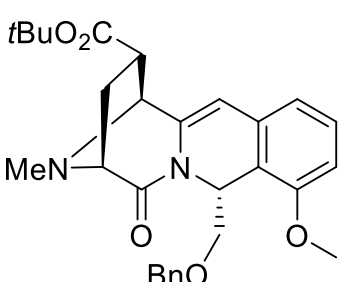

16
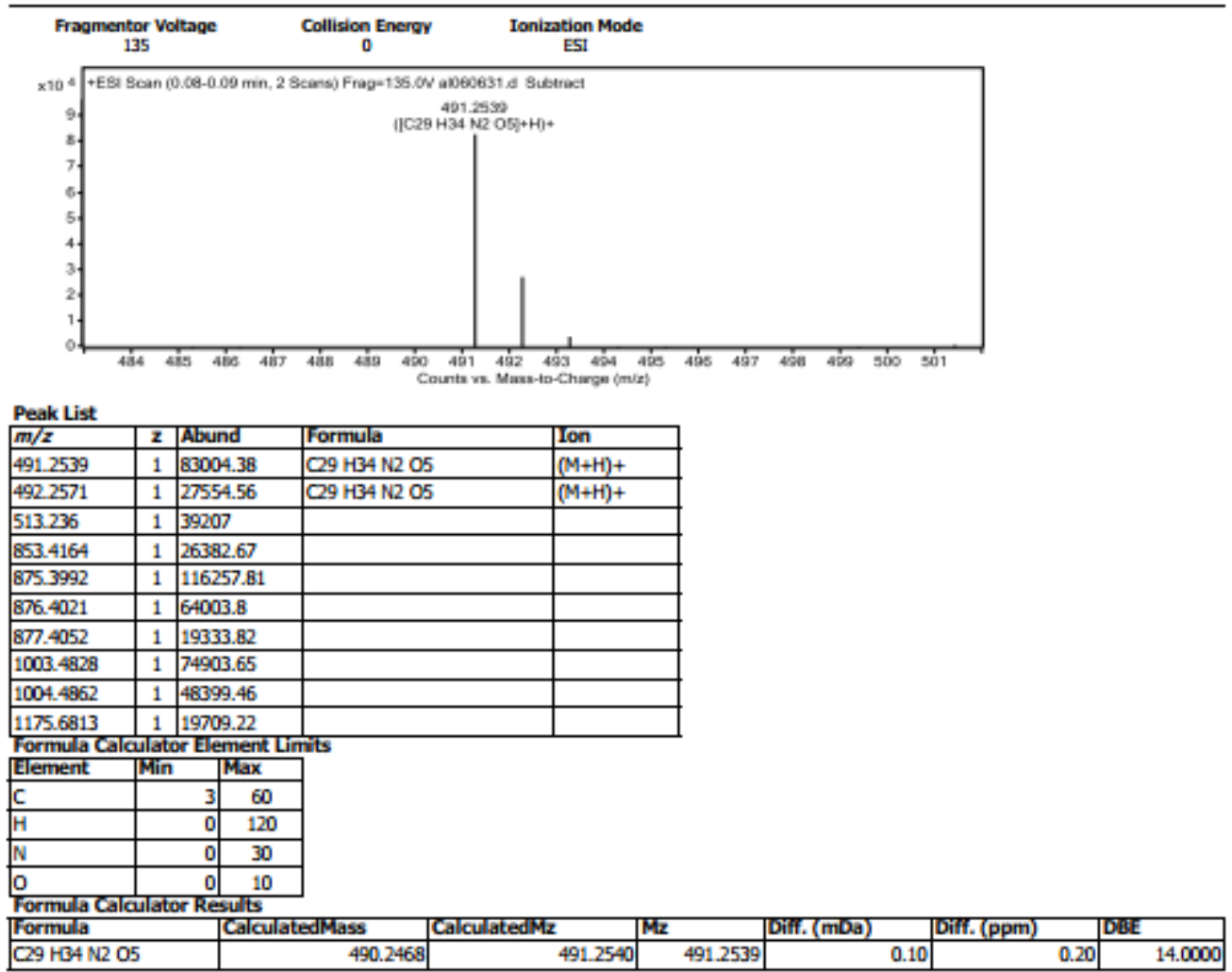

- End or Report - 


\section{Qualitative Analysis Report}

\begin{tabular}{|c|c|}
\hline Data Filename & alor107.d \\
\hline Sample Type & Sample \\
\hline Instrument Name & Instrument 1 \\
\hline Acq Method & $\mathrm{sm}$ \\
\hline $\begin{array}{l}\text { IRM Calibration Status } \\
\text { Comment }\end{array}$ & Success \\
\hline Sample Group & \\
\hline $\begin{array}{l}\text { Acquisition SW } \\
\text { Version }\end{array}$ & $\begin{array}{l}\text { ies TOF/6500 series } \\
.05 .01 \text { (B5125.2) }\end{array}$ \\
\hline
\end{tabular}

Sample Name alo710

Position P1-A2

User Name

Acquired Time $\quad 12 / 28 / 2020$ 2:38:36 PM

DA Method

Defaut.m

to.

Version Q.TOF B.05.01 (B5125.2)

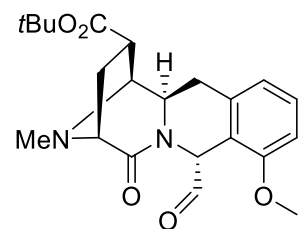

18

User Spectra

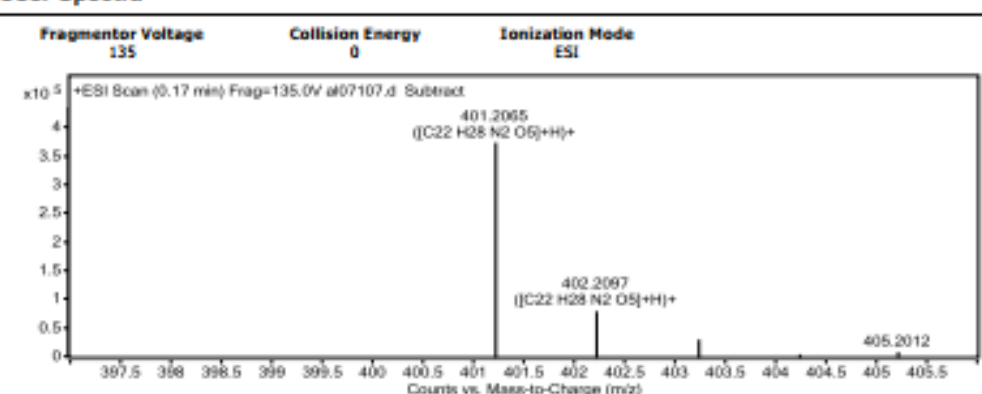

Peak List

Peak List
\begin{tabular}{|l|l|l|l|l|}
\hline$m / z$ & $z$ & Abund & Formula & Ion \\
\hline 345.1437 & 1 & 24089.72 & & \\
\hline 401.2065 & 1 & 374551.84 & $\mathrm{C2} 2 \mathrm{H} 28 \mathrm{~N} 2 \mathrm{OS}$ & $(\mathrm{M}+\mathrm{H})+$ \\
\hline 402.2097 & 1 & 82300.37 & $\mathrm{C2} \mathrm{H} 28 \mathrm{N2} \mathrm{OS}$ & $(\mathrm{M}+\mathrm{H})+$ \\
\hline 403.2194 & 1 & 32317.18 & $\mathrm{C2} 2 \mathrm{H} 28 \mathrm{~N} 2 \mathrm{OS}$ & $(\mathrm{M}+\mathrm{H})+$ \\
\hline 417.2012 & 1 & 39321.29 & & \\
\hline 423.1882 & 1 & 73609.13 & & \\
\hline 424.1905 & 1 & 17797.16 & & \\
\hline 433.2326 & 1 & 41836.52 & & \\
\hline 823.3892 & 1 & 63295.39 & & \\
\hline 824.3912 & 1 & 29600.13 & & \\
\hline
\end{tabular}

\begin{tabular}{llll}
2024.3912 & 1 & 29600.13 \\
\hline
\end{tabular}

Formula Calculator Element Limits

\begin{tabular}{|l|l|l|}
\hline Element & Min & Max \\
\hline
\end{tabular}

\begin{tabular}{|l|r|r|}
\hline$C$ & 3 & 120 \\
\hline
\end{tabular}

\begin{tabular}{|c|c|c|}
\hline $\mathrm{H}$ & 0 & 240 \\
\hline $\mathrm{O}$ & 0 & 30 \\
\hline
\end{tabular}

\begin{tabular}{|l|r|r|}
\hline$N$ & 0 & 5 \\
\hline
\end{tabular}

\begin{tabular}{|c|c|c|c|c|c|c|}
\hline Formula & CalculstedMass & CalculatedMz & $\mu_{2}$ & DIff. (m0s) & Diff. $(\mathrm{ppm})$ & DEE \\
\hline $62 \mathrm{H} 28 \mathrm{N2}$ OS & 400.1998 & \begin{tabular}{rr|}
401.2071 \\
\end{tabular} & 401.2065 & 0.60 & 1.50 & 10.0000 \\
\hline
\end{tabular}

- End of Report -.- 
Data Fe: E.,DATAL2021 10115lab7149 bod
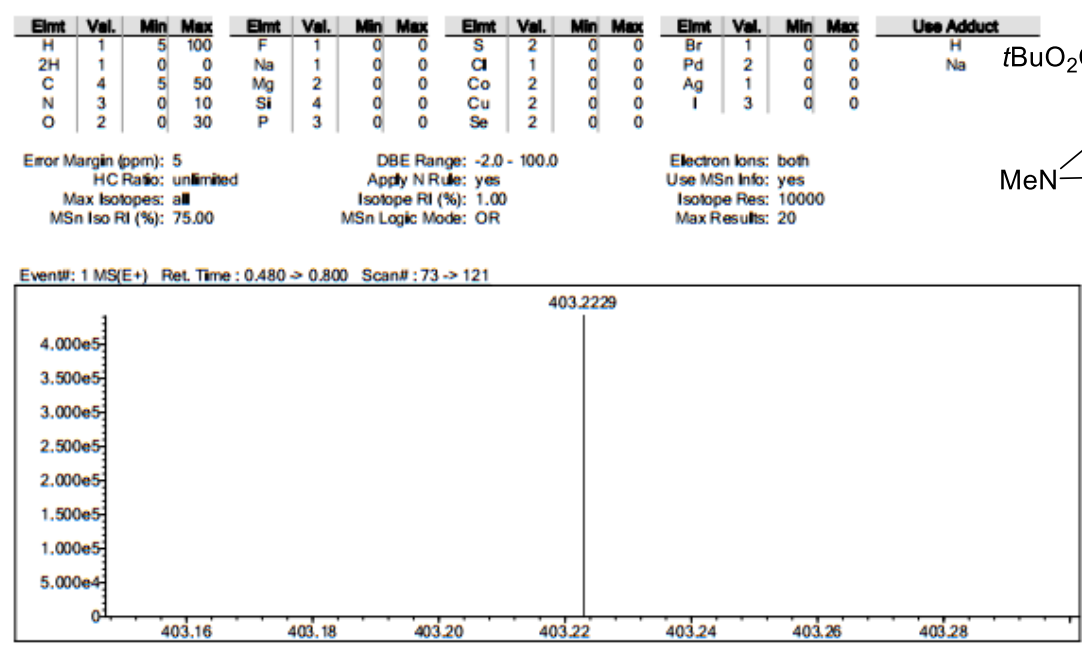

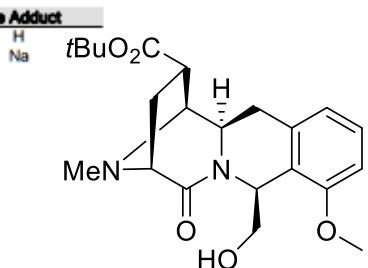

$\mathrm{HO} 20$

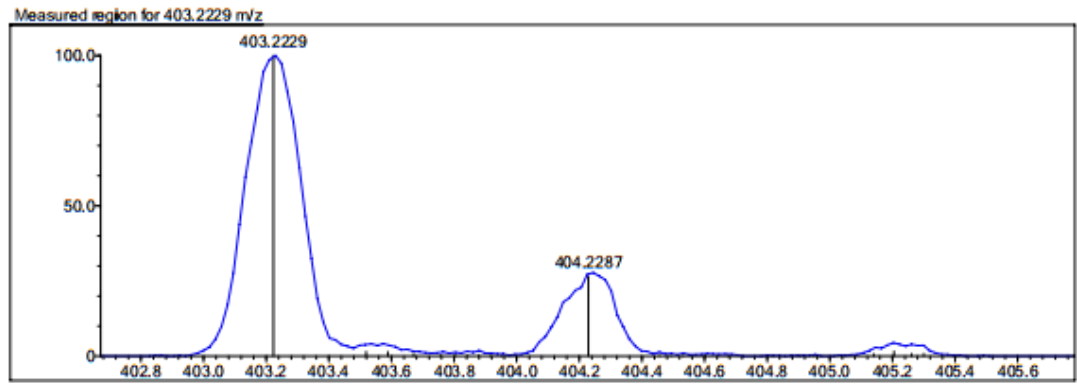

$\mathrm{C} 22 \mathrm{H} 30 \mathrm{~N} 2 \mathrm{O} 5[\mathrm{M}+\mathrm{H}]+$ : Predcled regon 1 bo $403.2227 \mathrm{~m} / 2$

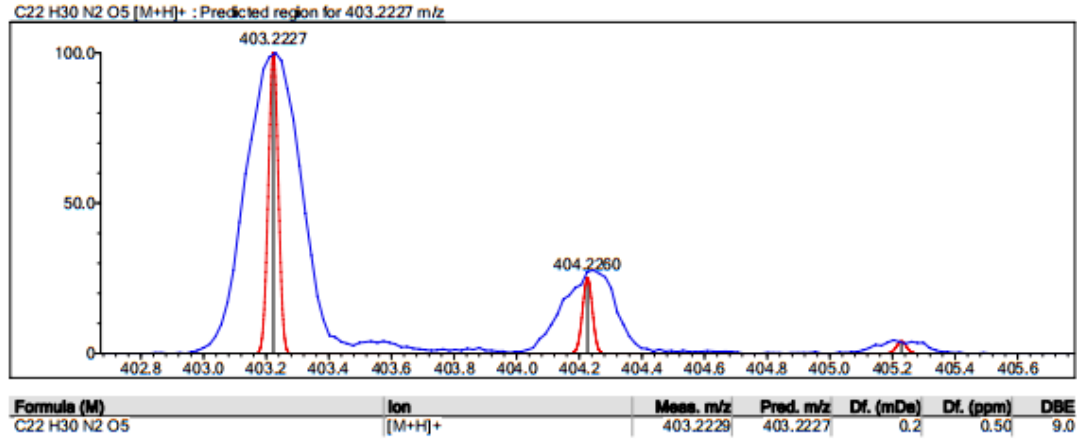


Qualitative Analysis Report

\begin{tabular}{|c|c|}
\hline Data Filename & aloea33.d \\
\hline Sample Type & Sample \\
\hline Instrument Name & Instrument 1 \\
\hline Acq Method & s.m \\
\hline $\begin{array}{l}\text { IRM Calibration Status } \\
\text { Comment }\end{array}$ & Success \\
\hline Sample Group & \\
\hline $\begin{array}{l}\text { Acquisition SW } \\
\text { Version }\end{array}$ & $\begin{array}{l}\text { ies TOF/6500 series } \\
.05 .01 \text { (BS125.2) }\end{array}$ \\
\hline
\end{tabular}

Sample Name al00033

Position P1-A1

User Name $\quad \mathrm{HO}_{2} \mathrm{C}$

Acquired Time 2/26/2021 3:07:41 P94

DA Method Default.m<smiles>COc1cccc2c1[C@@H](CO)N1C(=O)[C@H]3C[C@@H]([C@@H]3CC(=O)O)[C@H]21</smiles>

$(+)-3$

User Spectra

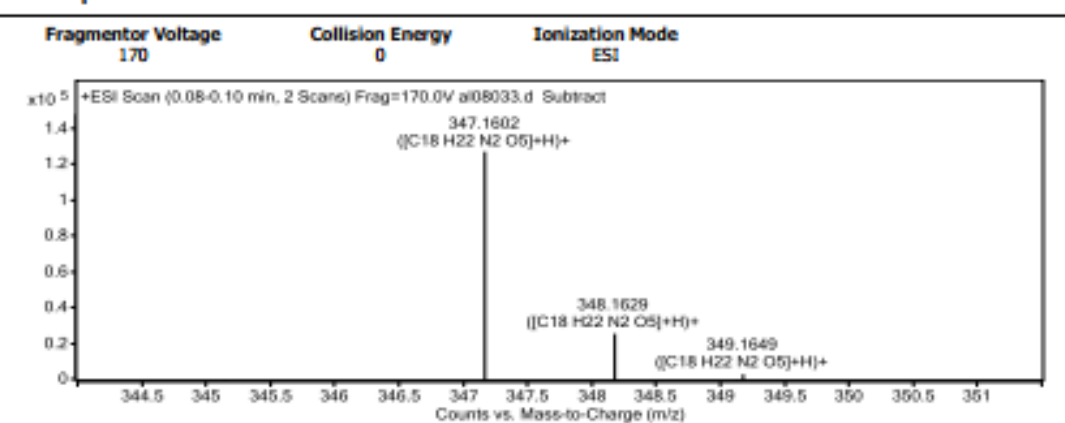

Peak List
\begin{tabular}{|l|r|l|l|l|}
\hline$m / z$ & $z$ & Abund & Formula & Ion \\
\hline 347.1602 & 1 & 127536.35 & C18 H22 N2 O5 & $(\mathrm{M}+\mathrm{H})+$ \\
\hline 348.1629 & 1 & 26282.88 & $\mathrm{C} 18 \mathrm{H} 22 \mathrm{~N} 2$ O5 & $(\mathrm{M}+\mathrm{H})+$ \\
\hline 403.2233 & 1 & 309063.03 & & \\
\hline 404.2255 & 1 & 74406.31 & & \\
\hline 413.2658 & 1 & 87445.73 & & \\
\hline 419.217 & 1 & 29659.09 & & \\
\hline 425.2039 & 1 & 63680.88 & & \\
\hline 793.4987 & 1 & 63936.12 & & \\
\hline 794.502 & 1 & 32227.26 & & \\
\hline 803.5422 & 1 & 40849.77 & & \\
\hline 7
\end{tabular}

Formula Calculator Element Lim

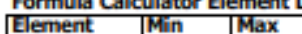

\begin{tabular}{|l|c|c|}
\hline Element & Min & Max \\
\hline $\mathrm{C}$ & 3 & 60 \\
\hline $\mathrm{H}$ & 0 & 120 \\
\hline $\mathrm{O}$ & 0 & 30 \\
\hline $\mathrm{N}$ & 0 & 3 \\
\hline
\end{tabular}

0 o 3

\begin{tabular}{|l|c|c|c|c|c|c|}
\hline Formula & CalculatedMass & CalculatedMz & Mz & Diff. (mba) & Diff. (ppm) & DBE \\
\hline C18 H22 N2 O5 & 346.1529 & 347.1601 & 347.1602 & -0.10 & -0.29 & 9.0000 \\
\hline
\end{tabular}

-- End or Report - 


\section{Crystal data for compound 11}

$$
\mathrm{C}_{14} \mathrm{H}_{20} \mathrm{BrNO}_{4}, M=346.22, a=10.9179(5) \AA, b=10.9179(5) \AA, c=22.7941(10) \AA, \alpha=90^{\circ}, \beta=
$$

$90^{\circ}, \gamma=120^{\circ}, V=2353.1(2) \AA^{3}, T=100$.(2) $\mathrm{K}$, space group $P 3221, Z=6, \mu(\mathrm{Cu} \mathrm{K \alpha})=3.687 \mathrm{~mm}^{-1}$, 30756 reflections measured, 3112 independent reflections $\left(R_{\text {int }}=0.0706\right)$. The final $R_{l}$ values were $0.0392(I>2 \sigma(I))$. The final $w R\left(F^{2}\right)$ values were $0.0941(I>2 \sigma(I))$. The final $R_{I}$ values were 0.0393 (all data). The final $w R\left(F^{2}\right)$ values were 0.0941 (all data). The goodness of fit on $F^{2}$ was 1.190. Flack parameter $=0.177(14)$.

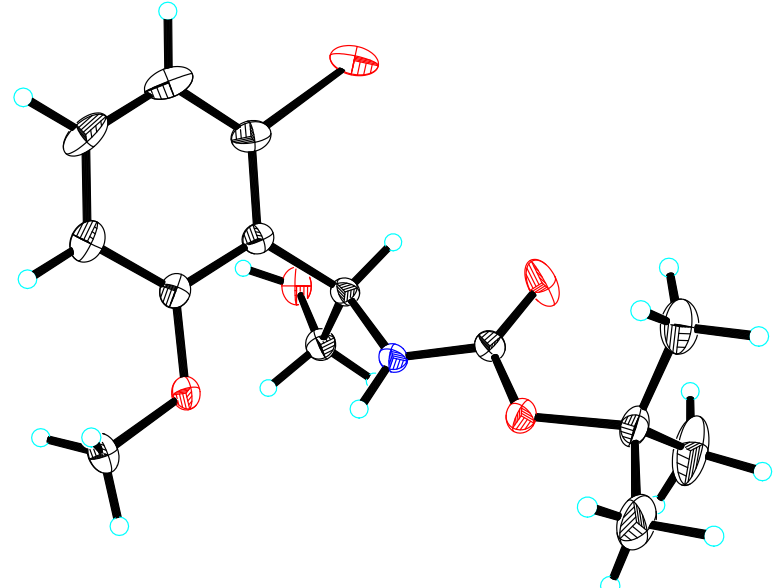

Displacement ellipsoids are drawn at the $30 \%$ probability level.

\section{Table 1. Crystal data and structure refinement for compound 11.}

Identification code

Empirical formula

Formula weight

Temperature

Wavelength

Crystal system

Space group

Unit cell dimensions

Volume

Z global

C14 H20 Br N O4

346.22

100(2) K

$1.54178 \AA$

Trigonal

$\mathrm{P}_{2} 21$

$\mathrm{a}=10.9179(5) \AA \quad=90^{\circ}$.

$\mathrm{b}=10.9179(5) \AA \quad=90^{\circ}$.

$\mathrm{c}=22.7941(10) \AA \quad=120^{\circ}$.

2353.1(2) $\AA^{3}$

6 
Density (calculated)

Absorption coefficient

$\mathrm{F}(000)$

Crystal size

Theta range for data collection

Index ranges

Reflections collected

Independent reflections

Completeness to theta $=72.03^{\circ}$

Absorption correction

Max. and min. transmission

Refinement method

Data / restraints / parameters

Goodness-of-fit on $\mathrm{F}^{2}$

Final $\mathrm{R}$ indices [I $>2 \operatorname{sigma}(\mathrm{I})]$

$\mathrm{R}$ indices (all data)

Absolute structure parameter

Largest diff. peak and hole
$1.466 \mathrm{Mg} / \mathrm{m}^{3}$

$3.687 \mathrm{~mm}^{-1}$

1068

$0.320 \times 0.200 \times 0.120 \mathrm{~mm}^{3}$

4.68 to $72.03^{\circ}$.

$-13<=\mathrm{h}<=13,-13<=\mathrm{k}<=13,-28<=1<=28$

30756

$3112[\mathrm{R}(\mathrm{int})=0.0706]$

$100.0 \%$

Semi-empirical from equivalents

0.67 and 0.36

Full-matrix least-squares on $\mathrm{F}^{2}$

3112 / 0 / 186

1.190

$\mathrm{R} 1=0.0392, \mathrm{wR} 2=0.0941$

$\mathrm{R} 1=0.0393, \mathrm{wR} 2=0.0941$

$0.177(14)$

0.498 and -0.526 e. $\AA^{-3}$ 